Supporting Information of

\title{
Semisynthesis of Intact Complex-Type Triantennary Oligosaccharides from a Biantennary Oligosaccharide Isolated from a Natural Source by Selective Chemical and Enzymatic Glycosylation
}

\author{
Yuta Maki, ${ }^{1}$ Ryo Okamoto, ${ }^{1}$ Masayuki Izumi, ${ }^{1}$ Takefumi Murase, ${ }^{2}$ and Yasuhiro Kajihara* ${ }^{1}$ \\ ${ }^{1}$ Department of Chemistry, Graduate School of Science, Osaka University, 1-1 Machikaneyama-cho, \\ Toyonaka, Osaka 560-0043, Japan \\ ${ }^{2}$ International Graduate School of Arts and Science, Yokohama City University, 22-2 Seto, Kanazawa-ku, \\ Yokohama, Kanagawa 236-0027, Japan
}




\section{Table of Contents:}

1. General Methods

2. Experimental Procedures and Characterizations

2.1 Synthesis of Glycosyl Donor 4

2.2 Synthesis of a Triantennary Oligosaccharide Having $\beta$-1,6-Branching 6

2.3 Synthesis of a Triantennary Oligosaccharide Having $\beta$-1,4-Branching 21

2.4 Synthesis of Benzylidene Monosaccharides 29

2.5 Investigation of Acidic Hydrolysis of Benzylidene Groups 30

3. Figures

3.1 Figure S1.

3.2 Figure S2.

3.3 Figure S3.

3.4 Figure S4.

3.5 Figure S5.

3.6 Figure S6. 36

3.7 Figure S7.

3.8 Figure S8.

3.9 Figure S9.

3.10 Figure S10.

3.11 Figure S11.

3.12 Figure S12.

3.13 Figure S13.

3.14 Figure S14.

4. Examples of other investigation of the reactions

5. NMR Data

6. References

65 


\section{General Methods}

${ }^{1} \mathrm{H}$ and ${ }^{13} \mathrm{C}$ NMR spectra were recorded on a $400 \mathrm{MHz}$ or $700 \mathrm{MHz}$ spectrometer (Bruker Avance III). All ${ }^{1} \mathrm{H}$ chemical shifts are reported in parts per million (ppm) relative to TMS in $\mathrm{CDCl}_{3}(0 \mathrm{ppm}), \mathrm{H}_{2} \mathrm{O}$ in $\mathrm{CD}_{3} \mathrm{CN}$ (2.13 ppm), $\mathrm{CD}_{3} \mathrm{CN}$ (1.94 ppm), or $\mathrm{D}_{2} \mathrm{O}(4.79 \mathrm{ppm})$. All ${ }^{13} \mathrm{C}$ chemical shifts are assigned according to $\mathrm{CD}_{3} \mathrm{Cl}$ (77.16 ppm), $\mathrm{CD}_{3} \mathrm{CN}$ (1.32 or $113.26 \mathrm{ppm}$ ), d3-AcOD (2.03), or 1,4-dioxane in $\mathrm{D}_{2} \mathrm{O}$ as the external standard (67.19 ppm). All NMR signals were assigned on the basis of ${ }^{1} \mathrm{H}$ NMR, DQF-COSY, HSQC, HMBC, and HSQC-TOCSY. High-resolution mass spectrometry was performed by FTICR (Bruker solariX XR), and other mass spectra were recorded on a Bruker esquire $3000^{\text {plus }}$, or an amaZon ETD mass spectrometer. TLC-analysis was conducted on Silicagel $60 \mathrm{~F}_{254}$ (Merck TLC plates), and visualizations were performed with UV light $(254 \mathrm{~nm})$ and/or sulfuric acid stain $\left(5 \% \mathrm{H}_{2} \mathrm{SO}_{4}\right.$ in Methanol). RP-HPLC analyses were performed using $0.1 \%$ aq. TFA and $90 \%$ aq. $\mathrm{CH}_{3} \mathrm{CN}$ containing $0.1 \%$ TFA, and RP-UHPLC analyses were performed using $0.1 \%$ aq. $\mathrm{HCOOH}$ and $90 \%$ aq. $\mathrm{CH}_{3} \mathrm{CN}$ containing $0.09 \%$ HCOOH. RP-HPLC analyses were performed on a Waters 2996 HPLC system equipped with a multi wavelength detector, and RP-UHPLC analyses were performed on a DIONEX UltiMate 3000 UHPLC system equipped with a variable wavelength detector. An $\mathrm{XBridge}^{\mathrm{TM}}$ prep column (Waters, $10 \times 250 \mathrm{~mm}$ ) was used for preparative HPLC using $50 \mathrm{mM}$ aq. $\mathrm{NH}_{4} \mathrm{OAc}$ and $\mathrm{CH}_{3} \mathrm{CN}$. A Cadenza CD-C18 (Imtakt, $4.6 \mathrm{~mm} \times 75 \mathrm{~mm}$ ) column or a Proteonavi (Shiseido, $4.6 \times 250 \mathrm{~mm}$ ) column were used for analytical HPLC using $0.1 \%$ aq. TFA and $90 \%$ aq. $\mathrm{CH}_{3} \mathrm{CN}$ containing $0.1 \%$ TFA, or $50 \mathrm{mM}$ aq. $\mathrm{NH}_{4} \mathrm{OAc}$ and $\mathrm{CH}_{3} \mathrm{CN}$, respectively. 


\section{Experimental Procedures and Characterizations}

\subsection{Synthesis of Glycosyl Donor}

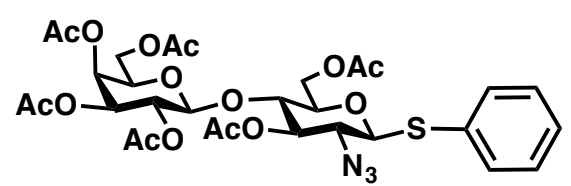

Phenyl $\quad(2,3,4,6$-tetra- $O$-acetyl- $\beta$-D-galactopyranosyl)-(1 $\rightarrow 4)$-3,6-di- $O$-acetyl-2-azido-2deoxy-1-thio- $\beta$-D-glucopyranoside (S1).

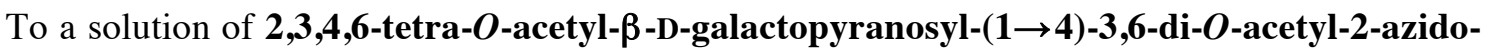
2-deoxy-a-D-glucopyranosyl chloride ${ }^{1,2}(1.913 \mathrm{~g}, 3.00 \mathrm{mmol})$ in ethylacetate $(24 \mathrm{~mL})$ was added tetrabutylammonium hydrogensulfate $(1.013 \mathrm{~g}, 2.98 \mathrm{mmol})$, thiophenol $(911 \mu \mathrm{L}, 8.93$ $\mathrm{mmol})$, and $1 \mathrm{M}$ aqueous sodium carbonate $(24 \mathrm{~mL})$. The resulting mixture was stirred at $40{ }^{\circ} \mathrm{C}$ for 2 hours $^{3}$. The mixture was diluted with ethylacetate, washed by $0.1 \mathrm{M}$ aq. $\mathrm{NaHCO}_{3}$ twice, dried over $\mathrm{Mg}_{2} \mathrm{SO}_{4}$, filtered, and concentrated. The resulting residue was purified by silica gel column chromatography (hexane $/$ EtOAc $=5: 1$ to $1: 1, \phi=5.0 \mathrm{~cm}, 1=15 \mathrm{~cm}$ ). Fractions containing the desired thioglycoside were combined and evaporated. The resulting residue was recrystallized from ethanol and petroleum ether to give $\mathbf{S 1}$ (539 $\mathrm{mg}, 0.757 \mathrm{mmol}, 25 \%)$.

$[\alpha]_{D}{ }^{17}-32.3\left(c 0.42 \mathrm{CHCl}_{3}\right)$

${ }^{1}$ H NMR (400 MHz, $\mathrm{CDCl}_{3}$, TMS): 7.60-7.54 (m, $\left.2 \mathrm{H}\right), 7.39-7.31(\mathrm{~m}, 3 \mathrm{H}), 5.35$ (brd, $1 \mathrm{H}, J=$ $3.29 \mathrm{~Hz}), 5.13-5.04(\mathrm{~m}, 2 \mathrm{H}), 4.94(\mathrm{dd}, J=10.31,3.42 \mathrm{~Hz}, 1 \mathrm{H}), 4.52(\mathrm{dd}, J=12.00,1.41 \mathrm{~Hz}, 1$ H), $4.47(\mathrm{~d}, J=10.13 \mathrm{~Hz}, 1 \mathrm{H}), 4.44(\mathrm{~d}, J=7.91 \mathrm{~Hz}, 1 \mathrm{H}), 4.16(\mathrm{dd}, J=11.04,6.18 \mathrm{~Hz}, 1 \mathrm{H})$, $4.11(\mathrm{dd}, J=11.98,4.96 \mathrm{~Hz}, 1 \mathrm{H}), 4.11(\mathrm{dd}, J=7.49,11.08 \mathrm{~Hz}, 1 \mathrm{H}), 3.86$ (bddd $J=6.72 \mathrm{~Hz}, 1$ H), 3.67-3.56 (m, 2 H), 3.32 (dd, $J=10.07,10.02 \mathrm{~Hz}, 1 \mathrm{H}), 2.15$ (s, $3 \mathrm{H}), 2.12(\mathrm{~s}, 3 \mathrm{H}), 2.11$ (s, $3 \mathrm{H}), 2.04$ (s, $3 \mathrm{H}), 2.03$ (s, $3 \mathrm{H}), 1.96$ (s, $3 \mathrm{H})$.

${ }^{13}$ C NMR (100 MHz, $\left.\mathrm{CDCl}_{3}\right): 20.64,20.74,20.77,20.98,60.93,62.15,63.24,66.73,69.21$, 70.86, 71.12, 73.93, 75.87, 77.36, 77.01, 86.01, 101.07, 128.88, 129.17, 130.81, 134.06, 169.03, $169.49,170.21,170.26,170.35,170.43$.

HRMS (ESI): calcd for $\mathrm{C}_{30} \mathrm{H}_{37} \mathrm{Cl}_{3} \mathrm{O}_{17} \mathrm{~S}[\mathrm{M}+\mathrm{Na}]^{+} 734.1838$, found for $m / z 734.1834[\mathrm{M}+\mathrm{Na}]^{+}$. 


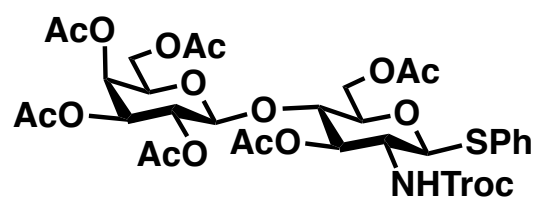

Phenyl $\quad(2,3,4,6$-tetra- $O$-acetyl- $\beta$-D-galactopyranosyl)-(1 $\rightarrow 4)$-3,6-di- $O$-acetyl-2-deoxy-2(2,2,2-trichloroethoxycarbonylamino)-1-thio- $\beta$-D-glucopyranoside (10).

To a solution of $\mathbf{S 1}(530 \mathrm{mg}, 0.74 \mathrm{mmol})$ in tetrahydrofuran $(7.4 \mathrm{~mL})$ was slowly added AcOH $(2.2 \mathrm{~mL}, 37 \mathrm{mmol})$ and activated zinc $(4.8 \mathrm{~g}, 74 \mathrm{mmol})$. The reaction mixture was stirred at $\mathrm{rt}$ for 30 min and diluted with $\mathrm{CH}_{2} \mathrm{Cl}_{2}$. The resulting mixture was filtered over celite, washed by sat. aq. $\mathrm{NaHCO}_{3}$ thrice, dried over $\mathrm{MgSO}_{4}$, filtered, and concentrated in vacuo. Triethylamine $(267 \mu \mathrm{L}, 1.92 \mathrm{mmol})$ and 2,2,2-trichloroethyl chloroformate were slowly added to a solution of crude amine in $\mathrm{CH}_{2} \mathrm{Cl}_{2}(5.3 \mathrm{~mL})$ and stirred at $\mathrm{rt}$ under argon gas for 5 hours. This reaction mixture was diluted with $\mathrm{CH}_{2} \mathrm{Cl}_{2}$, washed by sat. aq. $\mathrm{NaHCO}_{3}$ thrice and Brine, dried over $\mathrm{MgSO}_{4}$, filtered, and concentrated. The crude reside was purified by silica gel column chromatography (hexane/EtOAc $=1: 2.5$ to $2: 3, \phi=3.5 \mathrm{~cm}, 1=15 \mathrm{~cm}$ ). Fractions containing the desired product were combined and evaporated. The resulting residue was recrystallized from ethanol and petroleum ether to give glycosyl donor $\mathbf{1 0}(224 \mathrm{mg}, 0.26 \mathrm{mmol}, 35 \%)^{4}$.

$[\alpha]_{\mathrm{D}}{ }^{17}-7.63\left(c 1.0 \mathrm{CHCl}_{3}\right)$

${ }^{1}$ H NMR (400 MHz, $\mathrm{CDCl}_{3}$, TMS): 7.52-7.45 (m, $\left.2 \mathrm{H}\right), 7.33-7.27$ (m, $\left.3 \mathrm{H}\right), 5.35$ (brd, $J=3.22$ $\mathrm{Hz}, 1 \mathrm{H}), 5.27$ (d, $J=9.57 \mathrm{~Hz}, 1 \mathrm{H}), 5.14(\mathrm{dd}, J=9.40,8.76 \mathrm{~Hz}, 1 \mathrm{H}), 5.10(\mathrm{dd}, J=10.30,7.85$ $\mathrm{Hz}, 1 \mathrm{H}), 4.96$ (dd, $J=10.44,3.37 \mathrm{~Hz}, 1 \mathrm{H}), 4.81$ (d, $J=12.06 \mathrm{~Hz}, 1 \mathrm{H}), 4.74$ (d, $J=12.12 \mathrm{~Hz}, 1$ H), $4.70(\mathrm{~d}, J=10.39 \mathrm{~Hz}, 1 \mathrm{H}), 4.55-4.45(\mathrm{~m}, 2 \mathrm{H}), 4.18-4.02(\mathrm{~m}, 3 \mathrm{H}), 3.87$ (brddd, $J=6.84$ Hz, $1 \mathrm{H}), 3.82-3.70$ (m, 2 H), 3.64 (ddd, $J=9.51,5.74,1.83 \mathrm{~Hz}, 1 \mathrm{H}), 2.13(\mathrm{~s}, 3 \mathrm{H}), 2.11$ (s, $3 \mathrm{H})$, 2.06 (s, 3 H), 2.05 (s, 3 H), 2.04 (s, 3 H), 1.97 (s, 3 H).

${ }^{13}$ C NMR (100 MHz, $\left.\mathrm{CDCl}_{3}\right): 20.65,20.75,20.78,20.94,20.98,55.35,60.98,62.44,66.76$, 69.24, 70.89, 71.07, 73.58, 74.74, 76.34, 77.36, 87.11, 95.53, 101.23, 128.29, 129.06, 132.46, $132.82,154.30,169.24,170.19,170.25,170.43,170.48,170.55$.

HRMS (ESI): calcd for $\mathrm{C}_{33} \mathrm{H}_{40} \mathrm{Cl}_{3} \mathrm{NO}_{17} \mathrm{~S}[\mathrm{M}+\mathrm{Na}]^{+}$882.0975; found for $m / z 882.0962[\mathrm{M}+\mathrm{Na}]^{+}$. 


\subsection{Synthesis of a Triantennary Oligosaccharide Having $\beta-1,6-B r a n c h i n g$}

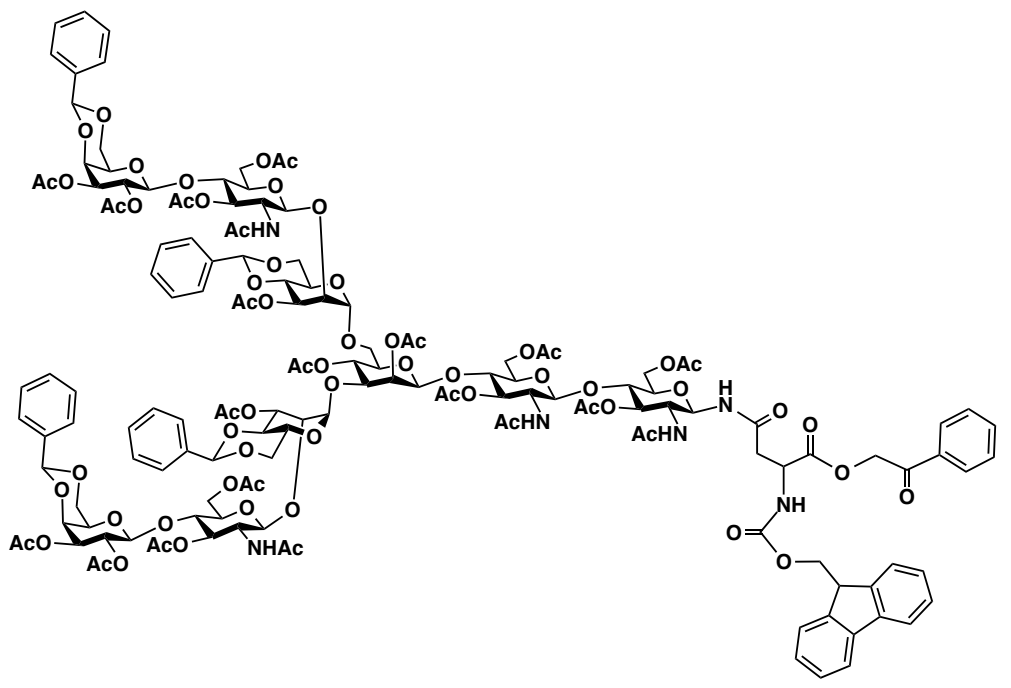

$N^{2}$-(9-Fluorenylmethyloxycarbonyl)- $N^{4}-\{O$-(2,3-di- $O$-acetyl-4,6-O-benzylidene- $\beta$-Dgalactopyranosyl)-(1 $\rightarrow 4)$ - $O$-(2-acetamido-3,6-di- $O$-acetyl-2-deoxy- $\beta$-D-glucopyranosyl)$(1 \rightarrow 2)-O$-(3-O-acetyl-4,6-O-benzylidene- $\alpha$-D-mannopyranosyl)-( $1 \rightarrow 3)$ - $O$-[(2,3-di- $O$-acetyl4,6-O-benzylidene- $\beta$-D-galactopyranosyl)-(1 $\rightarrow 4)$ - $O$-(2-acetamido-3,6-di- $O$-acetyl-2-deoxy$\beta$-D-glucopyranosyl)-(1 $\rightarrow 2)-O$-(3-O-acetyl-4,6-O-benzylidene- $\alpha$-D-mannopyranosyl)$(1 \rightarrow 6)]-O$ - $(2,4-d i-O$-acetyl- $\beta$-D-mannopyranosyl)-(1 $\rightarrow 4)$ - $O$-(2-acetamido-3,6-di- $O$-acetyl-2deoxy- $\beta$-D-glucopyranosyl)-( $1 \rightarrow 4)$-(2-acetamido-3,6-di- $O$-acetyl-2-deoxy- $\beta$-Dglucopyranosyl)\}-L-asparagine phenacyl ester (5).

To a solution of Asn-linked biantennary asialo-nonasaccharide $\mathbf{3}^{\mathbf{5}}$ (139 $\mathrm{mg}, 70.4 \mu \mathrm{mol}$ ) in DMF (14 mL) were added camphor-10-sulfonic acid (162 mg, 697 umol) and benzaldehyde dimethyl acetal ( $420 \mu \mathrm{L}, 2.71 \mathrm{mmol})$. The reaction mixture was stirred under an atomosphere of Ar at rt, and the reaction was monitored with RP-HPLC. After 17 hours, the reaction mixture was poured into ice-cold diethylether to form a precipitate. The suspension was centrifuged, and diethylether was decanted. The precipitate was then washed twice more with ice-cold diethylether and air-dried to give crude tetra-benzylidene derivative 4 . The resultant $\mathbf{4}$ was then used for the next reaction without further purification; ESI-MS calcd for $\mathrm{C}_{109} \mathrm{H}_{136} \mathrm{~N}_{6} \mathrm{O}_{50}$ $[\mathrm{M}+2 \mathrm{H}]^{2+} 1165.4$, found for $m / z$ 1165.4.

To a solution of crude tetra-benzylidene derivative 4 in DMF $(14 \mathrm{~mL})$ were added $N, N$ '-diisopropylethylamine (62 uL, $354 \mu \mathrm{mol})$ and 2-bromoacetophenone (42 mg, $211 \mu \mathrm{mol})$. The reaction mixture was stirred at $\mathrm{rt}$ for 3 hours, and RP-HPLC showed complete conversion to the phenacyl ester (S2). The mixture was treated with ice-cold diethylether and centrifuged to 
give crude ester S2. The resulting S2 was then used for the next reaction without further purification; ESI-MS calcd for $\mathrm{C}_{117} \mathrm{H}_{142} \mathrm{~N}_{6} \mathrm{O}_{51}[\mathrm{M}+2 \mathrm{H}]^{2+} 1224.4$, found for $m / z$ 1224.5.

To a solution of crude ester $\mathbf{S} \mathbf{2}$ in acetic anhydride $(1.4 \mathrm{~mL})$ and pyridine $(1.4 \mathrm{~mL})$, $N, N$ '-dimethyl-4-aminopyridine (9 $\mathrm{mg}, 70 \mathrm{umol}$ ) was added, and the resulting mixture was stirred at rt for 3 hours. The reaction was monitored with RP-HPLC and TLC. After the completion of the reaction, ice-cold $\mathrm{MeOH}$ was added to the mixture on an ice bath. The mixture was then allowed to warm up to rt, after which it was concentrated, and the resulting residue was azeotropically dried with toluene several times. The resulting residue was dissolved in aq. $\mathrm{CH}_{3} \mathrm{CN}$, filtered, and purified by preparative $\mathrm{RP}-\mathrm{HPLC}$ (XBridge, $50 \mathrm{mM}$ aq. $\mathrm{NH}_{4} \mathrm{OAc}$ : $\mathrm{CH}_{3} \mathrm{CN}=40: 60$ to $30: 70$ over $90 \mathrm{~min}$ at $4 \mathrm{~mL} / \mathrm{min}$ ). Fractions containing the desired product were combined, treated with Dowex to remove ammonium acetate salt, filtered, and lyophilized to provide fully protected oligosaccharide 5 (52 $\mathrm{mg}, 24 \%$ ) as a white form.

$[\alpha]_{\mathrm{D}}{ }^{24}-10.3\left(c: 0.93, \mathrm{CHCl}_{3}\right)$

${ }^{1}$ H NMR (400 MHz, CD 3 CN, HDO 2.13 ppm) $\delta 7.52(\mathrm{~d}, J=7.75 \mathrm{~Hz}, 2 \mathrm{H}), 7.42(\mathrm{~d}, J=7.49 \mathrm{~Hz}$, 2 H), 7.29-7.20 (m, 3 H), 7.18-7.09 (m, 3 H), 7.09-6.88 (m, 23 H), 6.34-6.25 (m, 2 H), 6.25-6.18 (m, $2 \mathrm{H}), 6.04$ (d, $J=8.61 \mathrm{~Hz}, 1 \mathrm{H}), 5.18(\mathrm{~s}, 1 \mathrm{H}), 5.14$ (s, $3 \mathrm{H}), 5.06-4.94(\mathrm{~m}, 3 \mathrm{H})$, $4.89(\mathrm{dd} J=9.95,9.85 . \mathrm{Hz}, 1 \mathrm{H}), 4.78-4.54(\mathrm{~m}, 10 \mathrm{H}), 4.47(\mathrm{~s}, 1 \mathrm{H}), 4.45(\mathrm{dd}, J=6.21,3.19 \mathrm{~Hz}$, $1 \mathrm{H}), 4.39$ (s, $1 \mathrm{H}), 4.33(\mathrm{~s}, 1 \mathrm{H}), 4.33-4.28(\mathrm{~m}, 1 \mathrm{H}), 4.23-4.15(\mathrm{~m}, 3 \mathrm{H}), 4.09$ (d, J = 8.39 Hz, 1 H), $4.03(\mathrm{~d}, J=8.61 \mathrm{~Hz}, 1 \mathrm{H}), 4.01-3.65(\mathrm{~m}, 19 \mathrm{H}), 3.64-3.41(\mathrm{~m}, 9 \mathrm{H}), 3.41-3.10(\mathrm{~m}, 17 \mathrm{H})$, 2.45-2.30 (m, 2 H), 1.75 (s, 3 H), 1.72 (s, 3 H), 1.69 (s, 3 H), 1.69 (s, 6 H), 1.67 (s, 3 H), 1.66 (s, $3 \mathrm{H}), 1.65$ (s, $3 \mathrm{H}), 1.62(\mathrm{~s}, 3 \mathrm{H}), 1.61(\mathrm{~s}, 3 \mathrm{H}), 1.60$ (s, $3 \mathrm{H}), 1.58(\mathrm{~s}, 9 \mathrm{H}), 1.56(\mathrm{~s}, 6 \mathrm{H}), 1.54$ (s, $3 \mathrm{H}), 1.46$ (s, $3 \mathrm{H}), 1.45$ (s, $3 \mathrm{H}), 1.43(\mathrm{~s}, 3 \mathrm{H}), 1.36$ (s, $3 \mathrm{H})$.

${ }^{13}$ C NMR (100 MHz, $\mathrm{CD}_{3} \mathrm{CN} 118.26$ ppm) $\delta$ 193.32, 171.97, 171.84, 171.57, 171.54, 171.47, $171.41,171.21,171.17,171.13,171.08,171.02$, 170.91, 170.57, 170.54, 170.49, 156.88, 145.01, $144.09,139.28$, 139.25, 138.74, 135.01, 134.98, 130.04, 129.97, 129.87, 129.19, 129.13, 129.06 , $128.74,128.68,128.12$, 127.41, 127.32, 127.25, 126.14, 120.95, 102.75, 102.55, 101.64, 101.55, $101.32,101.02$, 100.60, 99.68, 99.21, 79.52, 79.10, 78.49, 77.84, 77.61, 76.78, 76.42, 76.37, $76.14,75.61,75.55,75.26,74.43,74.37,74.01,73.78,73.68,73.54,73.29,73.00,72.84,72.22$, $71.63,70.31,69.97,69.92,69.87,69.34,69.21,69.13,68.38,67.94,67.42,67.19,67.11,65.65$, $64.91,63.45,63.35,63.25,62.24,55.00,54.90,54.15,53.78,51.51,47.93,38.26,23.24,23.18$, $23.05,21.57,21.54,21.22,21.19,21.16,21.12,21.08,21.05,21.01,20.98,20.89$. 
HRMS (ESI): calcd for $\mathrm{C}_{149} \mathrm{H}_{174} \mathrm{~N}_{6} \mathrm{O}_{67}[\mathrm{M}+2 \mathrm{H}]^{2+} 1560.5269$, found for $\mathrm{m} / \mathrm{z} 1560.5242$ $[\mathrm{M}+2 \mathrm{H}]^{2+}$.

Assignment: HSQC of peracetate $5(400 \mathrm{MHz})$.

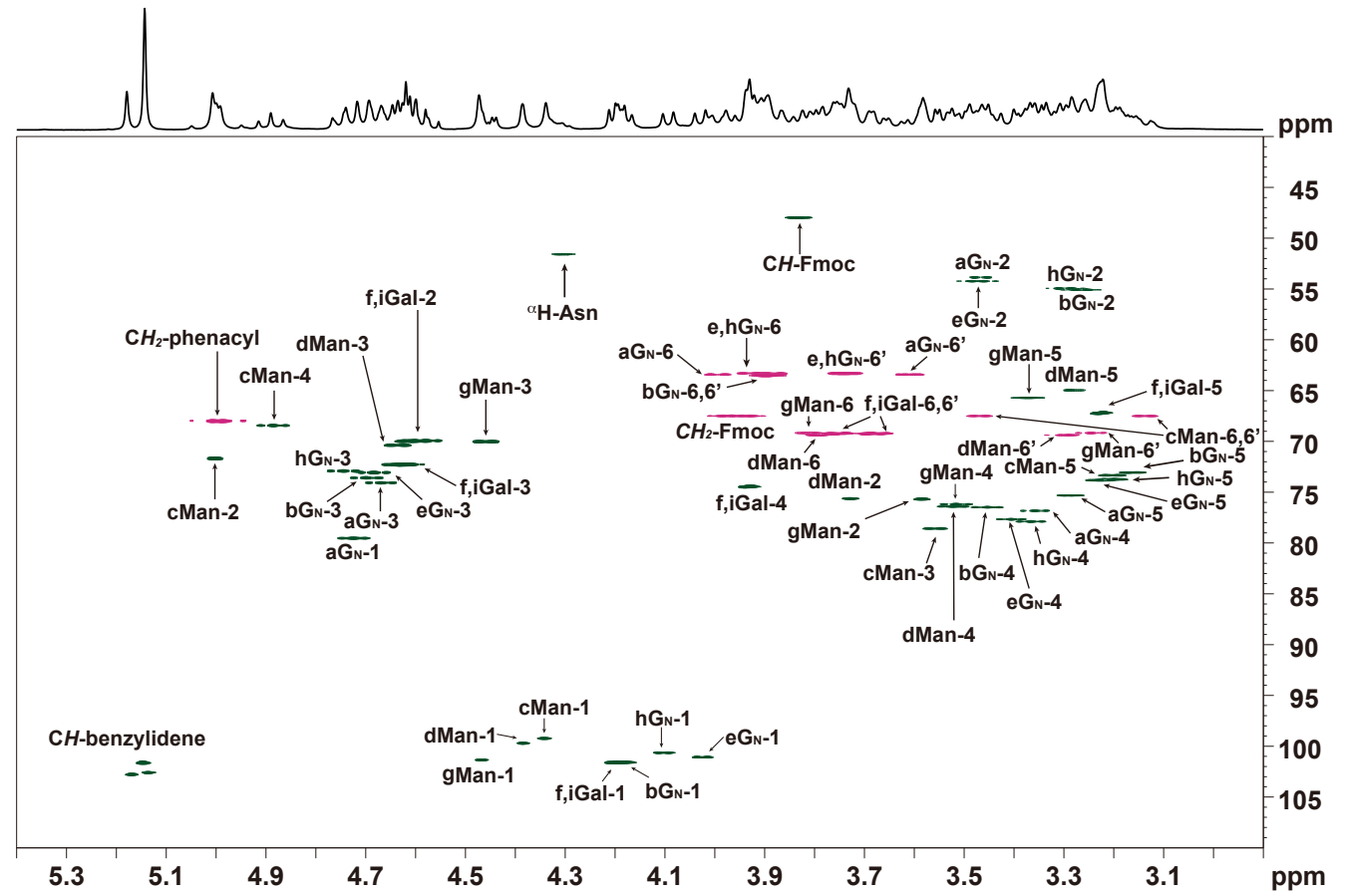

All chemical shifts were assigned by combined 2D NMR experiments. 


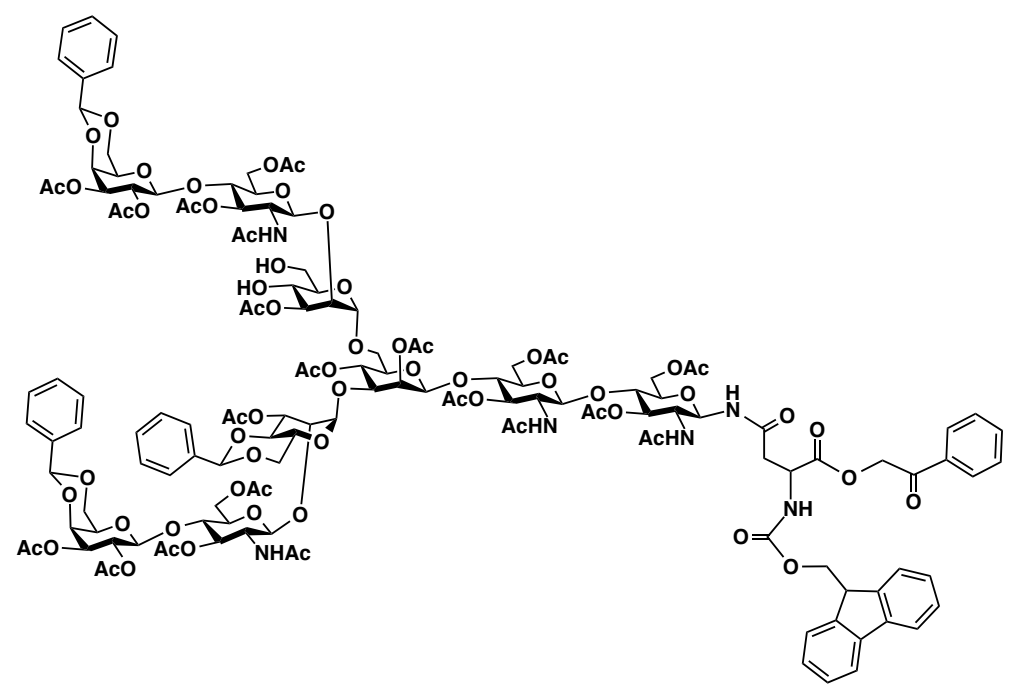

$N^{2}$-(9-Fluorenylmethyloxycarbonyl)- $N^{4}-\{O$-(2,3-di- $O$-acetyl-4,6- $O$-benzylidene- $\beta$-Dgalactopyranosyl)-(1 $\rightarrow 4)$ - $O$-(2-acetamido-3,6-di- $O$-acetyl-2-deoxy- $\beta$-D-glucopyranosyl)$(1 \rightarrow 2)-O$-(3-O-acetyl-4,6- $O$-benzylidene- $\alpha$-D-mannopyranosyl)-(1 $\rightarrow 3)-O$-[(2,3-di- $O$-acetyl4,6- $O$-benzylidene- $\beta$-D-galactopyranosyl)-(1 $\rightarrow 4)$ - $O$-(2-acetamido-3,6-di- $O$-acetyl-2-deoxy$\beta$-D-glucopyranosyl)-(1 $\rightarrow 2)-O$-(3-O-acetyl- $\alpha$-D-mannopyranosyl)-(1 $\rightarrow 6)]-O$-(2,4-di- $O$ acetyl- $\beta$-D-mannopyranosyl)-( $1 \rightarrow 4)-O$-(2-acetamido-3,6-di- $O$-acetyl-2-deoxy- $\beta$-Dglucopyranosyl)-(1 $\rightarrow 4)$-(2-acetamido-3,6-di- $O$-acetyl-2-deoxy- $\beta$-D-glucopyranosyl)\}-Lasparagine phenacyl ester (6).

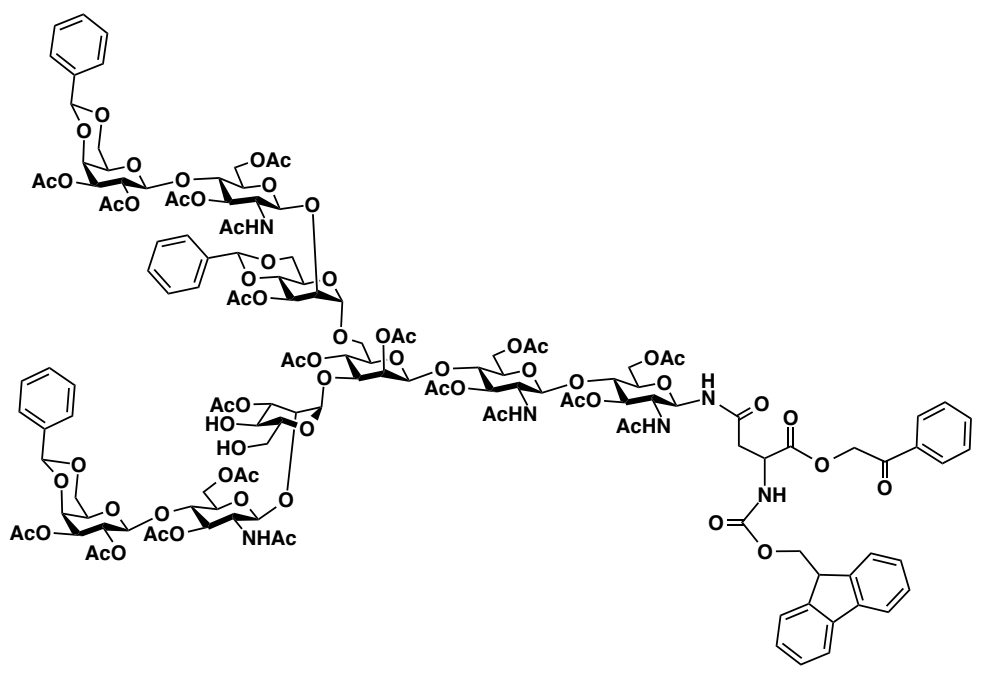

$N^{2}$-(9-Fluorenylmethyloxycarbonyl)- $N^{4}-\{O$-(2,3-di- $O$-acetyl-4,6-O-benzylidene- $\beta$-Dgalactopyranosyl)-(1 $\rightarrow 4)$ - $O$-(2-acetamido-3,6-di- $O$-acetyl-2-deoxy- $\beta$-D-glucopyranosyl)$(1 \rightarrow 2)$ - $O$-(3-O-acetyl- $\alpha$-D-mannopyranosyl)-(1 $\rightarrow 3)$ - $O$-[(2,3-di- $O$-acetyl-4,6-O-benzylidene- 
$\beta$-D-galactopyranosyl)-( $1 \rightarrow 4)-O$-(2-acetamido-3,6-di- $O$-acetyl-2-deoxy- $\beta$-D-glucopyranosyl )-(1 $\rightarrow 2)-O$-(3-O-acetyl-4,6-O-benzylidene- $\alpha$-D-mannopyranosyl)-(1 $\rightarrow 6)]-O-(2,4-d i-O$ acetyl- $\beta$-D-mannopyranosyl)-( $\rightarrow 4)-O$-(2-acetamido-3,6-di- $O$-acetyl-2-deoxy- $\beta$-Dglucopyranosyl)-( $1 \rightarrow 4)$-(2-acetamido-3,6-di- $O$-acetyl-2-deoxy- $\beta$-D-glucopyranosyl)\}-Lasparagine phenacyl ester (7).

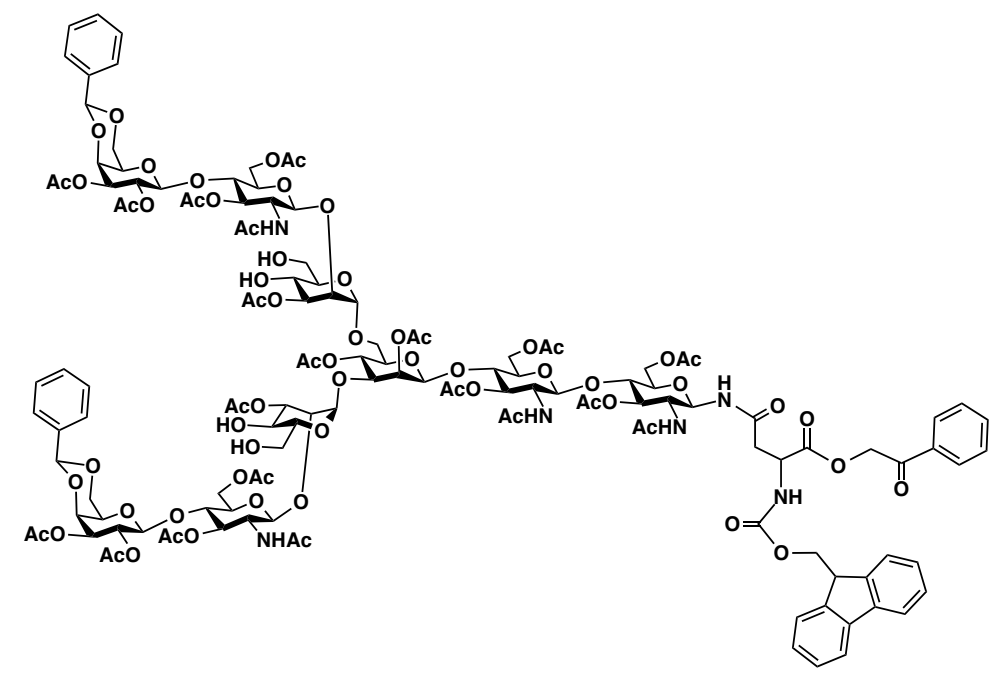

$N^{2}$-(9-Fluorenylmethyloxycarbonyl)- $N^{4}-\{O$-(2,3-di- $O$-acetyl-4,6-O-benzylidene- $\beta$-Dgalactopyranosyl)-(1 $\rightarrow 4)$ - $O$-(2-acetamido-3,6-di- $O$-acetyl-2-deoxy- $\beta$-D-glucopyranosyl)$(1 \rightarrow 2)-O$ - $(3-O$-acetyl- $\alpha$-D-mannopyranosyl)-(1 $\rightarrow 3)-O$-[(2,3-di- $O$-acetyl-4,6-O-benzylidene$\beta$-D-galactopyranosyl)-( $1 \rightarrow 4)-O$-(2-acetamido-3,6-di- $O$-acetyl-2-deoxy- $\beta$-D-glucopyranosyl )-(1 $\rightarrow 2)-O$-(3-O-acetyl- $\alpha-D-m a n n o p y r a n o s y l)-(1 \rightarrow 6)]-O$ - $(2,4-d i-O$-acetyl- $\beta$-Dmannopyranosyl)-(1 $\rightarrow 4)$ - $O$-(2-acetamido-3,6-di- $O$-acetyl-2-deoxy- $\beta$-D-glucopyranosyl)$(1 \rightarrow 4)$-(2-acetamido-3,6-di- $O$-acetyl-2-deoxy- $\beta$-D-glucopyranosyl)\}-L-asparagine phenacyl ester (8).

The fully protected oligosaccharide 5 (21.3 mg, $6.83 \mathrm{umol}$ ) was dissolved in ice-cold $60 \%$ aq. $\mathrm{AcOH}$ and stirred with allowing the temperature to arise from $0{ }^{\circ} \mathrm{C}$ to rt. The reaction was monitored with RP-HPLC and TLC. After 10 hours, the mixture was then concentrated in vacuo and azeotropically dried with toluene. The resulting residue was dissolved in aq. $\mathrm{CH}_{3} \mathrm{CN}$, filtered, and purified by preparative HPLC (X-Bridge, $50 \mathrm{mM}$ aq. $\mathrm{NH}_{4} \mathrm{Ac}: \mathrm{CH}_{3} \mathrm{CN}=47: 53$ to 37: 63 over $90 \mathrm{~min}$ at $4 \mathrm{~mL} / \mathrm{min}$ ). Fractions containing di-benzylidene derivative 8 , 
tri-benzylidene derivatives 6 and 7, and remaining starting material 5 were individually collected, and then treated with Dowex in order to remove ammonium acetate salt. Lyophilization gave di-benzylidene derivative 8 (1.8 mg, 9\%), tri-benzylidene derivative 6 (2.7 $\mathrm{mg}, 13 \%)$, tri-benzylidene derivative 7 (3.3 mg, 15\%), and starting material 5 (3.4 $\mathrm{mg}, 16 \%)$ as white forms. The recovered starting material was used in deprotection of benzylidene acetal group repetitively. Benzylidene acetalation was performed to the di-benzylidene derivative by benzylidene dimethyl acetal and camphor-10-suphonic acid in $\mathrm{CH}_{3} \mathrm{CN}$ to yield tetra- and tri-benzylidene derivatives again.

Tri-benzylidene derivative $\mathbf{6}$;

${ }^{1}$ H NMR (400 MHz, CD ${ }_{3}$ CN, HOD 2.13 ppm) $\delta$ 7.79-7.71 (d, $\left.J=7.42 \mathrm{~Hz}, 2 \mathrm{H}\right)$, 7.69-7.63 (dd, $J=7.42,2.78 \mathrm{~Hz}, 2 \mathrm{H}), 7.51-7.46(\mathrm{~m}, 3 \mathrm{H}), 7.39-7.32(\mathrm{~m}, 2 \mathrm{H}), 7.31-7.11(\mathrm{~m}, 19 \mathrm{H}), 6.51-6.41(\mathrm{~m}$, $2 \mathrm{H}), 6.39-6.33(\mathrm{~d}, J=9.29 \mathrm{~Hz}, 1 \mathrm{H}), 6.22-6.14(\mathrm{~d}, J=8.77 \mathrm{~Hz}, 1 \mathrm{H}), 5.39-5.37$ (s, 2H), 5.37-5.35 (s, 1H), 5.31-5.16 (m, 3H), 5.02-4.93 (m, 2H), 4.93-4.77 (m, 8H), 4.73-4.69 (s, 1H), 4.69-4.62 $(\mathrm{m}, 2 \mathrm{H}), 4.59-4.50(\mathrm{~m}, 3 \mathrm{H}), 4.47-4.40(\mathrm{~m}, 2 \mathrm{H}), 4.39-4.31(\mathrm{~m}, 2 \mathrm{H}), 4.27-3.87(\mathrm{~m}, 18 \mathrm{H})$, 3.87-3.80 (m, 4H), 3.80-3.56 (m, 12H), 3.56-3.31 (m, 12H), 2.69-2.52 (m, 2H), 2.00-1.97 (s, $3 \mathrm{H}), 1.94-1.92(\mathrm{~s}, 6 \mathrm{H}), 1.91-1.89$ (s, 6H), 1.89 (s, 3H), 1.89-1.87 (s, 6H), 1.86-1.84 (s, 3H), $1.84-1.82(\mathrm{~s}, 9 \mathrm{H}), 1.81-1.80(\mathrm{~s}, 3 \mathrm{H}), 1.80-1.79(\mathrm{~s}, 6 \mathrm{H}), 1.79-1.78(\mathrm{~s}, 3 \mathrm{H}), 1.70-1.68(\mathrm{~s}, 6 \mathrm{H})$, $1.68-1.66(\mathrm{~s}, 3 \mathrm{H}), 1.64-1.59(\mathrm{~s}, 3 \mathrm{H})$.

${ }^{13}$ C NMR (100 MHz, CD ${ }_{3}$ CN, 118.26 ppm) $\delta$ 193.27, 172.49, 172.09, 171.98, 171.82, 171.56, $171.49,171.45,171.22$, 171.11, 171.06, 170.94, 170.89, 170.55, 170.50, 156.85, 144.98, 142.06, 139.26, 139.23, 138.70, 134.97, 130.02, 129.96, 129.85, 129.18, 129.05, 128.72, 128.66, 128.09, 127.28, 127.24, 126.13, 120.94, 102.52, 101.97, 101.59, 101.50, 101.10, 100.48, 98.77, 98.34, 79.53, 77.92, 77.79, 77.38, 76.85, 76.10, 75.67, 75.47, 75.13, 74.39, 74.35, 74.08, 73.94, 73.79, 73.68, 73.59, 73.45, 72.96, 72.77, 72.73, 72.18, 71.37, 69.92, 69.86, 69.81, 69.11, 68.92, 67.92, $67.51,67.39,67.15,67.08,65.62,64.60,63.40,63.29,63.22,62.42,54.87,54.73,54.20,53.68$, $51.45,47.89,38.21,23.46,23.32,23.21,23.13,23.03,21.54,21.49,21.25,21.17,21.14,21.11$, $21.03,20.98,20.94,20.88,20.60$.

HRMS (ESI): calcd for $\mathrm{C}_{142} \mathrm{H}_{170} \mathrm{~N}_{6} \mathrm{O}_{67}[\mathrm{M}+2 \mathrm{H}]^{2+} 1516.5113$, found for $\mathrm{m} / z 1516.5156$ $[\mathrm{M}+2 \mathrm{H}]^{2+}$.

Tri-benzylidene derivative 7;

${ }^{1}$ H NMR (400 MHz, CD $\left.\mathbf{C N}\right) \delta 7.80(\mathrm{~d}, J=7.46 \mathrm{~Hz}, 2 \mathrm{H}), 7.69(\mathrm{~d}, J=7.54 \mathrm{~Hz}, 2 \mathrm{H})$, 
7.56-7.49(m, 3 H), 7.43-7.37 (m, 2 H), 7.37-6.15 (m, 19 H), 6.44-6.30 (m, 4 H), 6.17 (d, $J=$ 8.79, 1 H), 5.44 (s, 1 H), $5.42(\mathrm{~s}, 1 \mathrm{H}), 5.41(\mathrm{~s}, 1 \mathrm{H}), 5.33-5.19$ (m, $3 \mathrm{H}), 5.12$ (dd, $J=9.89,9.77$ Hz, 1 H), 5.03-4.80 (m, 10 H), 4.64 (s, 2 H), 4.61-4.53 (m, 2 H), 4.52-4.40 (m, 4 H), 4.31-3.84 (m, $21 \mathrm{H}), 3.84-3.35$ (m, $26 \mathrm{H})$, 2.71-2.55 (m, 2 H), 2.02 (s, $3 \mathrm{H}), 1.96$ (s, $3 \mathrm{H}), 1.95$ (s, $3 \mathrm{H})$, $1.92(\mathrm{~s}, 9 \mathrm{H}), 1.91(\mathrm{~s}, 6 \mathrm{H}), 1.87(\mathrm{~s}, 6 \mathrm{H}), 1.86(\mathrm{~s}, 3 \mathrm{H}), 1.85$ (s, $3 \mathrm{H}), 1.84(\mathrm{~s}, 6 \mathrm{H}), 1.82(\mathrm{~s}, 3 \mathrm{H})$, $1.73(\mathrm{~s}, 3 \mathrm{H}), 1.71(\mathrm{~s}, 6 \mathrm{H}), 1.68$ (s, $3 \mathrm{H}), 1.62(\mathrm{~s}, 3 \mathrm{H})$.

${ }^{13}$ C NMR (100 MHz, CD 3 CN, 118.26 ppm) $\delta$ 193.30, 171.97, 171.90, 171.83, 171.65, 171.57, $171.52,171.46,171.39,171.32,171.19,171.17,171.16,171.07,171.01,170.98,170.89$, $170.58,170.48,174.98,156.85,144.98,142.07,139.26,139.22,138.71,130.02,129.85$, $129.17,129.12,128.73,128.66,128.10,127.40,127.25,127.23,126.12,120.94,102.71$, $101.61,101.55,101.53,101.48,100.95,100.44,99.55,99.20,79.48,77.71,77.65,77.55,75.73$, $76.33,76.28,75.81,75.44,75.19,74.77,74.40,74.35,73.94,73.71,73.47,73.20,73.05,72.96$, 72.64, 72.19, 71.43, 70.30, 69.87, 69.81, 69.31, 69.18, 69.11, 68.46, 67.93, 67.39, 67.15, 67.08, $64.83,64.31,63.27,63.13,62.05,54.89,54.66,54.12,53.76,51.47,47.90,38.22,23.42,23.33$, 23.19, 23.15, 23.02, 21.58, 21.51, 21.22, 21.17, 21.14, 21.11, 21.06, 20.99, 20.95, 20.90, 20.87.

HRMS (ESI): calcd for $\mathrm{C}_{142} \mathrm{H}_{170} \mathrm{~N}_{6} \mathrm{O}_{67}[\mathrm{M}+2 \mathrm{H}]^{2+}$ 1516.5113, found for $\mathrm{m} / z 1516.5163$ $[\mathrm{M}+2 \mathrm{H}]^{2+}$.

Di-benzylidene derivative 8;

${ }^{1}$ H NMR (700 MHz, CD $\left.\mathbf{C N}\right) \delta 7.93(\mathrm{~d}, J=7.53 \mathrm{~Hz}, 2 \mathrm{H}), 7.83(\mathrm{~d}, J=7.56 \mathrm{~Hz}, 2 \mathrm{H})$, 7.69-7.64 (m, 3 H), 7.56-7.51 (m, 2 H), 7.46-7.39 (m, 12 H), 7.36-7.31 (m, 2 H), 7.24 (d, $J=$ $8.66 \mathrm{~Hz}, 1 \mathrm{H}), 6.51-6.46(\mathrm{~m}, 2 \mathrm{H}), 6.44(\mathrm{~d}, J=9.31 \mathrm{~Hz}, 1 \mathrm{H}), 6.40(\mathrm{~d}, J=9.11 \mathrm{~Hz}, 1 \mathrm{H}), 6.24(\mathrm{~d}$, $J=8.80 \mathrm{~Hz}, 1 \mathrm{H}), 5.55(\mathrm{~s}, 2 \mathrm{H}), 5.43(\mathrm{~d}, J=16.56 \mathrm{~Hz}), 5.37$ (d, $J=16.56 \mathrm{~Hz}, 1 \mathrm{H}), 5.34(\mathrm{~d}, J=$ $3.06 \mathrm{~Hz}, 1 \mathrm{H}), 5.14-4.94(\mathrm{~m}, 10 \mathrm{~Hz}), 4.80$ (dd, $J=9.89,3.27 \mathrm{~Hz}, 1 \mathrm{H}), 4.75$ (s, $1 \mathrm{H}), 4.73-4.64$ (m, $3 \mathrm{H}), 4.62-4.56(\mathrm{~m}, 3 \mathrm{H}), 4.54$ (d, $J=8.34 \mathrm{~Hz}, 1 \mathrm{H}), 4.42-4.29(\mathrm{~m}, 9 \mathrm{H}), 4.29-4.20(\mathrm{~m}, 3 \mathrm{H})$, 4.19-4.05 (m, $6 \mathrm{H}$ ), 4.02 (dd, $J=12.125 .23 \mathrm{~Hz}, 1 \mathrm{H}$ ), 3.98 (brs, $1 \mathrm{H}$ ), 3.95 (dd, $J=9.80,3.13$ Hz), 3.90-3.72 (m, $11 \mathrm{H}), 3.72-3.50(\mathrm{~m}, 15 \mathrm{H}), 3.47$ (dd, $J=10.53,2.44 \mathrm{~Hz}), 3.36$ (brs, $1 \mathrm{H})$, 3.17 (brs, $1 \mathrm{H}), 2.97$ (brs, $1 \mathrm{H}), 2.79$ (dd, $J=16.07,4.83 \mathrm{~Hz}, 1 \mathrm{H}), 2.73$ (dd, $J=16.12,6.44 \mathrm{~Hz}$, $1 \mathrm{H}), 2.13$ (s, $3 \mathrm{H}), 2.09$ (s, $6 \mathrm{H}), 2.08(\mathrm{~s}, 3 \mathrm{H}), 2.06$ (s, $3 \mathrm{H}), 2.05$ (s, $6 \mathrm{H}), 2.03$ (s, $3 \mathrm{H}), 2.02$ (s, $3 \mathrm{H}), 2.01$ (s, $6 \mathrm{H}), 2.00$ (s, $3 \mathrm{H}), 1.99$ (s, $3 \mathrm{H}), 1.96$ (s, $6 \mathrm{H}), 1.95$ (s, $3 \mathrm{H}), 1.85$ (s, $6 \mathrm{H}), 1.83$ (s, $3 \mathrm{H}), 1.75(\mathrm{~s}, 3 \mathrm{H})$.

${ }^{13}$ C NMR (100 MHz, CD ${ }_{3}$ CN 118.26 ppm) $\delta$ 193.28, 172.02, 171.95, 171.92, 171.82, 171.65, 171.52 , 171.48, 171.41, 171.38, 171.26, 171.23, 171.19, 170.99, 170.92, 170.88, 170.55, 170.55, 
$170.50,156.84,144.98,142.06,139.25,139.23,134.97,130.02,129.85,129.17,128.72,128.65$, $129.09,127.23,126.12,120.93,101.91,101.62$, 101.58, 101.51, 101.46, 100.30, 98.74, 98.47, 79.49, 77.53, 77.41, 77.21, 76.75, 75.69, 75.15, 74.99, 74.76, 74.38, 74.34, 74.10, 73.99, 73.86, $73.69,73.56,73.49,73.45,73.18,73.01,72.75,72.57,72.18,71.21,69.85,69.80,69.15,69.10$, $69.06,67.92,67.53,67.38,67.13,67.08,64.62,64.28,63.31,63.27,63.15,62.45,62.06,57.87$, $54.69,54.20,53.69,51.45,47.89,38.20,23.43,23.40,23.13,23.02,21.56,21.50,21.25,21.14$, $21.11,20.99,20.93,20.87,20.58,18.67$.

HRMS (ESI): calcd for $\mathrm{C}_{135} \mathrm{H}_{166} \mathrm{~N}_{6} \mathrm{O}_{67}[\mathrm{M}+2 \mathrm{H}]^{2+}$ 1472.4956, found for $\mathrm{m} / \mathrm{z} 11472.4956$ $[\mathrm{M}+2 \mathrm{H}]^{2+}$.

Assignment: HSQC of tri-benzylidene derivative 6 (700 MHz).

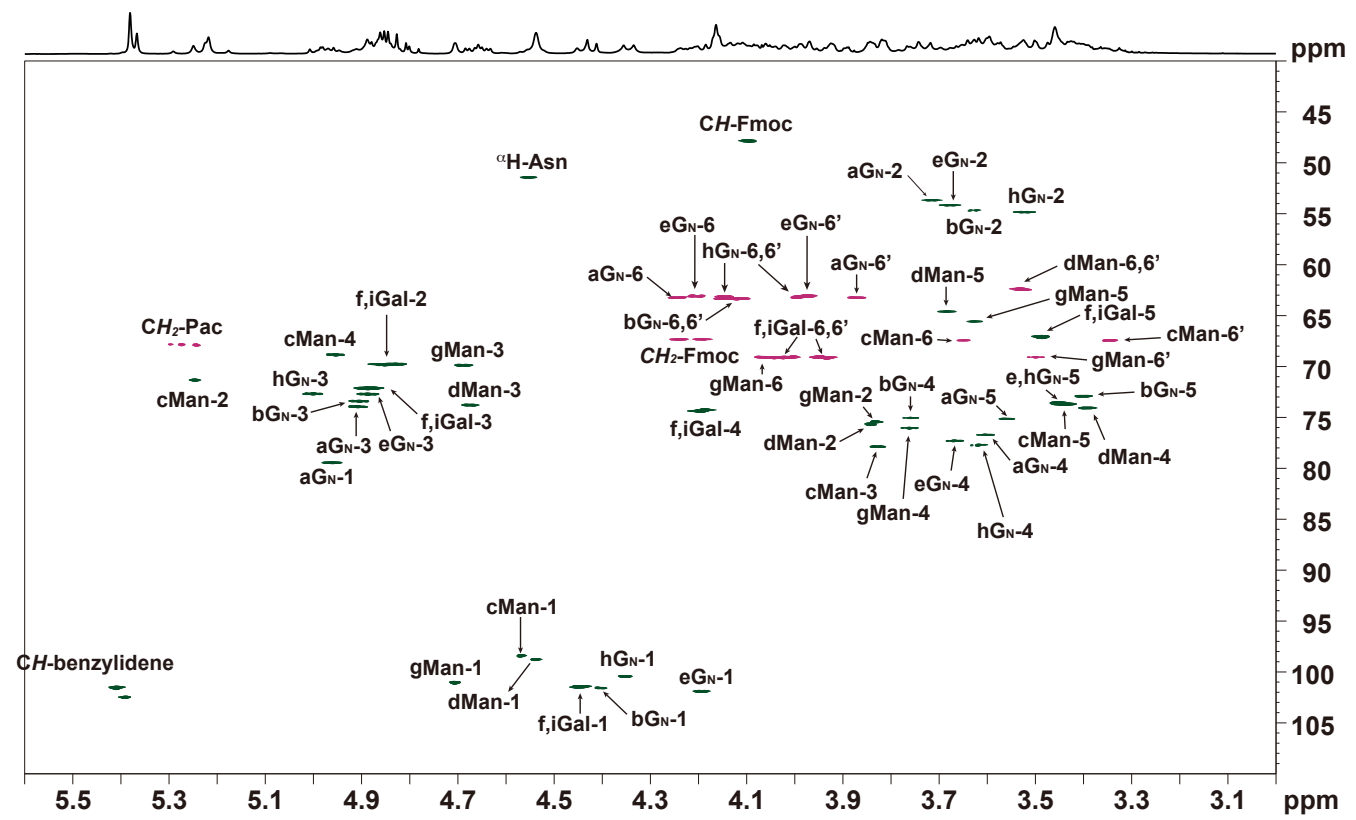

All chemical shifts were assigned by combined 2D NMR experiments. 
Assignment: HSQC of tri-benzylidene derivative 7 (400 MHz).

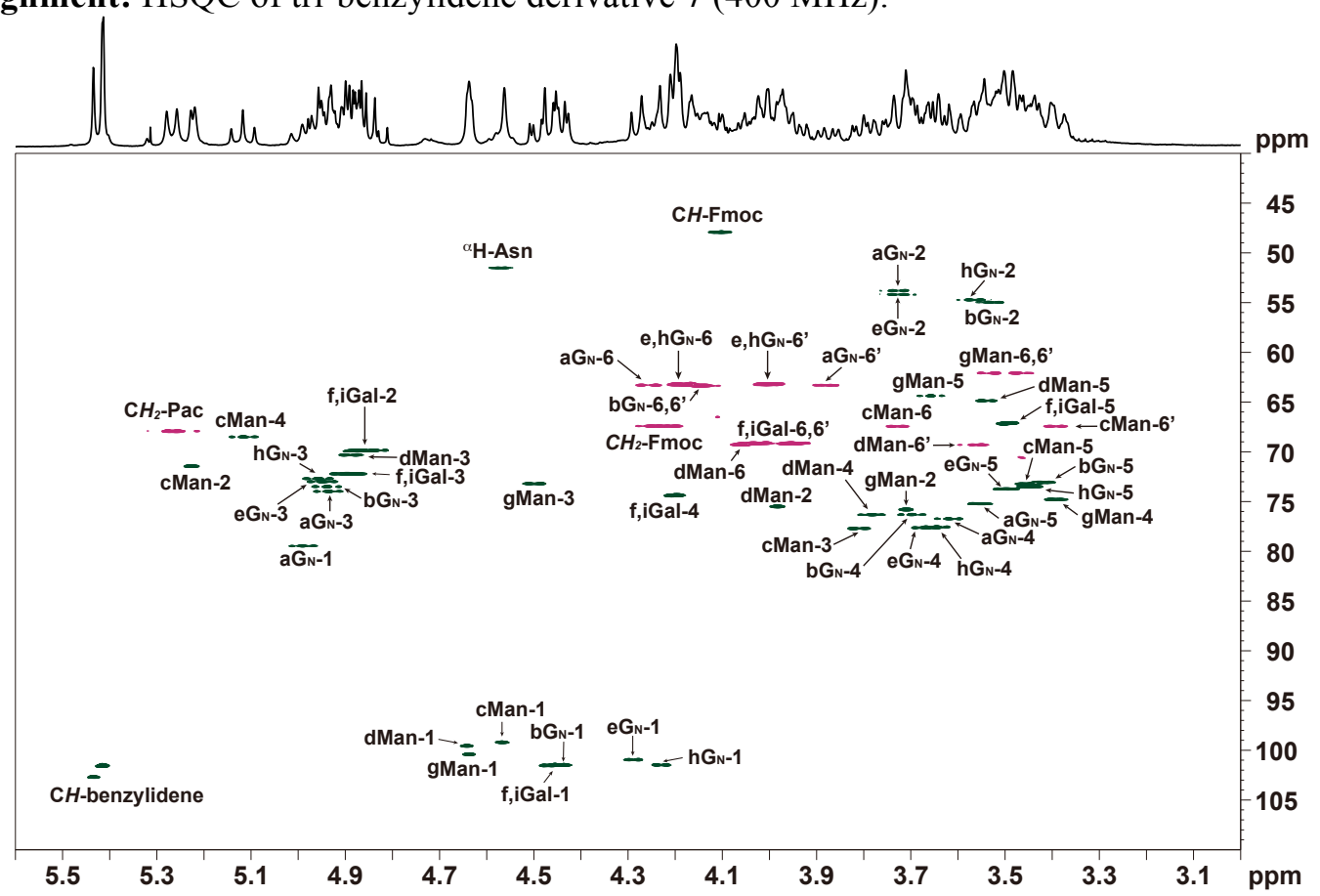

All chemical shifts were assigned by combined 2D NMR experiments.

Assignment: HSQC of di-benzylidene derivative 8 (700 MHz).

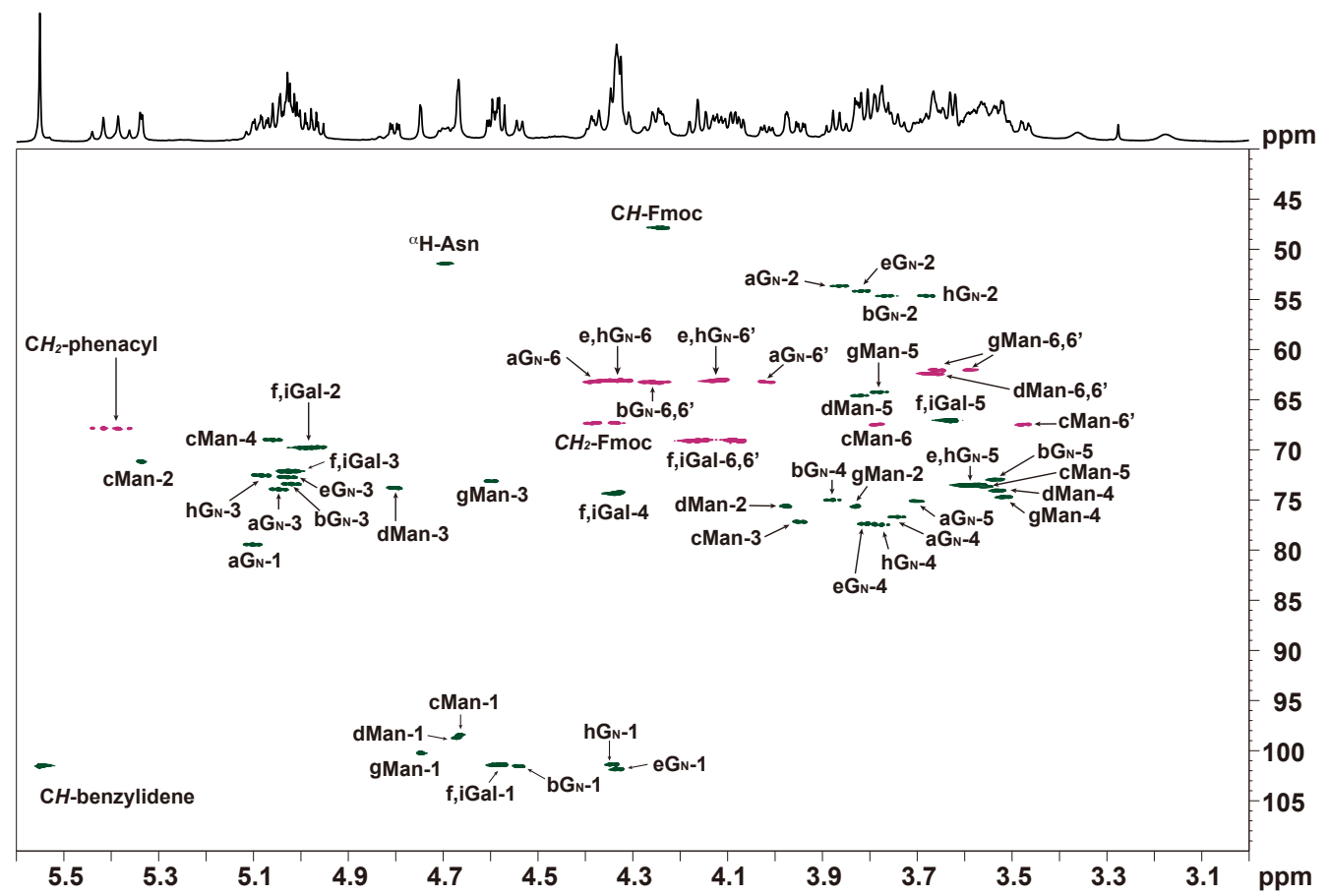

All chemical shifts were assigned by combined 2D NMR experiments. 


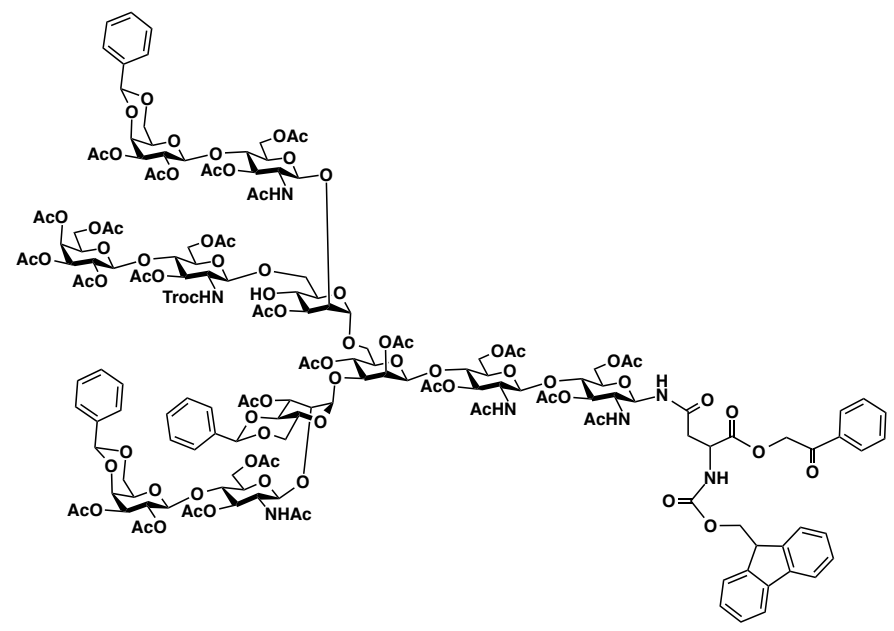

$N^{2}$-(9-Fluorenylmethyloxycarbonyl)- $N^{4}-\{O$-(2,3-di- $O$-acetyl-4,6-O-benzylidene- $\beta$-Dgalactopyranosyl)-(1 $\rightarrow 4)$ - $O$-(2-acetamido-3,6-di- $O$-acetyl-2-deoxy- $\beta$-D-glucopyranosyl)-(1 $\rightarrow 2)-O$-(3-O-acetyl-4,6-O-benzylidene- $\alpha$-D-mannopyranosyl)-( $\rightarrow 3)-O$-[(2,3-di- $O$-acetyl-4, 6- $O$-benzylidene- $\beta$-D-galactopyranosyl)-( $1 \rightarrow 4)$ - $O$-(2-acetamido-3,6-di- $O$-acetyl-2-deoxy- $\beta$ D-glucopyranosyl)-(1 $\rightarrow 2)$ - $O$-[(2,3,4,6-tetra- $O$-acetyl- $\beta$-D-galactopyranosyl)-(1 $\rightarrow 4)-(3,6$-di$O$-acetyl-2-deoxy-2-(2,2,2-trichloroethoxy)-carbonylamino- $\beta$-D-glucopyranosyl)-(1 $\rightarrow 6)]-(3$ - $O$-acetyl- $\alpha$-D-mannopyranosyl)-(1 $\rightarrow 6)]-O$-(2,4-di- $O$-acetyl- $\beta$-D-mannopyranosyl)-( $1 \rightarrow 4)$ $O$-(2-acetamido-3,6-di- $O$-acetyl-2-deoxy- $\beta$-D-glucopyranosyl)-(1 $\rightarrow 4)$-(2-acetamido-3,6-di$O$-acetyl-2-deoxy- $\beta$-D-glucopyranosyl)\}-L-asparagine phenacyl ester (11).

A mixture of acceptor $\mathbf{6}(8.4 \mathrm{mg}, 2.77 \mu \mathrm{mol})$ and donor $\mathbf{1 0}(23.9 \mathrm{mg}, 27.8 \mu \mathrm{mol}, 10$ equiv) was dried in vacuo for 3 hours, after which the mixture was dissolved in distilled $\mathrm{CH}_{2} \mathrm{Cl}_{2}(560 \mu \mathrm{L})$ under an atmosphere of Ar, followed by addition of freshly activated 4 A molecular sieves (56 $\mathrm{mg}$ ). The reaction mixture was cooled to $0{ }^{\circ} \mathrm{C}$, and NIS (5.0 mg, $22 \mathrm{umol}$ ) was added. Then, TfOH $\left(2.07 \mu \mathrm{mol}\right.$, with dilution by $\left.\mathrm{CH}_{2} \mathrm{Cl}_{2}\right)$ was added to the mixture, which was stirred for 1 hour at the same temperature. After the completion of the reaction, the mixture was diluted with chloroform, which was directly applied to silica gel column chromatography to remove a remaining glycosyl donor and its derivatives $(\phi 1.0 \times 7.0 \mathrm{~cm}$, Hexane: EtOAc (1: 1), EtOAc, EtOAc: $\mathrm{MeOH}(7: 1))$. The fractions containing oligosaccharides were combined and evaporated. The resulting residue was dissolved in aq. $\mathrm{CH}_{3} \mathrm{CN}$, filtered, and purified by preparative HPLC (XBridge, $50 \mathrm{mM}$ aq. $\mathrm{NH}_{4} \mathrm{OAc}$ : $\mathrm{CH}_{3} \mathrm{CN}=40: 60$ to 30: 70 over 90 min at 4 $\mathrm{mL} / \mathrm{min}$ ). Fractions containing the desired product were collected, treated with Dowex, filtered and lyophilized to give the protected triantennary oligosaccharide 11 (4.9 mg, 1.3 umol, 47\%) as a white form. 
${ }^{1}$ H NMR (400 MHz, CD 3 CN, HOD 2.13 ppm) $\delta 7.79$ (d, $\left.J=7.54 \mathrm{~Hz}, 2 \mathrm{H}\right), 7.69$ (d, $J=7.54$ Hz, 2 H), 7.55-7.49 (m, 3 H), 7.43-7.35 (m, 2 H), 7.35-7.16 (m, 19 H), 7.04 (d, J=7.96 Hz, 1 H), $6.53(\mathrm{~d}, J=9.65 \mathrm{~Hz}, 1 \mathrm{H}), 6.47-6.32(\mathrm{~m}, 3 \mathrm{H}), 6.18(\mathrm{~d}, J=8.68 \mathrm{~Hz}, 1 \mathrm{H}), 5.41(\mathrm{~m}, 2 \mathrm{H})$, $5.40(\mathrm{~s}, 1 \mathrm{H}), 5.33-5.20(\mathrm{~m}, 4 \mathrm{H}), 5.17(\mathrm{brd}, J=3.62 \mathrm{~Hz}, 1 \mathrm{H}), 5.04-4.77(\mathrm{~m}, 13 \mathrm{H}), 4.77-4.66(\mathrm{~m}$, $3 \mathrm{H}), 4.66-4.52(\mathrm{~m}, 5 \mathrm{H}), 4.39-4.32(\mathrm{~m}, 2 \mathrm{H}), 4.30(\mathrm{~d}, J=8.82 \mathrm{~Hz}, 1 \mathrm{H}), 4.27-3.81$ (m, $26 \mathrm{H})$, 3.81-3.29 (m, 26 H), 3.06 (dd, $J=18.95,8.58 \mathrm{~Hz}, 1 \mathrm{H}), 2.71-2.54$ (m, $2 \mathrm{H}), 1.99$ (s, $3 \mathrm{H}), 1.96$ (s, 9 H), 1.95 (s, 3 H), 1.92 (s, 3 H), 1.91 (s, 9 H), 1.90 (s, 3 H), 1.88 (s, 6 H), 1.87 (s, 3 H), 1.86 (s, 9 H), $1.82(\mathrm{~s}, 6 \mathrm{H}), 1.81$ (s, $3 \mathrm{H}), 1.79$ (s, $3 \mathrm{H}), 1.76(\mathrm{~s}, 3 \mathrm{H}), 1.74(\mathrm{~s}, 3 \mathrm{H}), 1.70$ (s, $6 \mathrm{H}), 1.62$ (s, $3 \mathrm{H})$.

Chemical shift of ${ }^{13} \mathrm{C}\left(\mathrm{CD}_{3} \mathrm{CN}\right.$, chemical shift picked up from HSQC except for quaternary carbon) $\delta 134.92,129.93,129.76,128.81,128.63,128.01,127.16,126.00,120.86,102.41$, $101.74,101.49,101.25,101.09,100.94,100.71,100.40,98.21,98.06,79.38,77.88,77.66$, $77.43,76.70,76.00,75.40,75.08,74.84,74.53,74.29,74.05,73.99,73.76,73.59,73.43,73.35$, $73.27,72.98,72.66,72.11,71.55,71.40,71.25,69.93,69.85,69.76,69.06,68.68,68.05,67.82$, $67.35,67.04,66.73,65.56,63.99,63.36,63.28,63.18,63.13,62.98,61.96,57.49,54.84,54.59$, 54.44, 53.60, 51.40, 47.81, 38.20, 23.46, 23.23, 23.02, 21.50, 21.17, 21.04, 20.88, 20.73.

HRMS (ESI): calcd for $\mathrm{C}_{169} \mathrm{H}_{204} \mathrm{Cl}_{3} \mathrm{~N}_{7} \mathrm{O}_{84}[\mathrm{M}+2 \mathrm{H}]^{2+} 1891.0559$, found for $\mathrm{m} / z 1891.0580$ $[\mathrm{M}+2 \mathrm{H}]^{2+}$.

Assignment: HSQC of $\beta-1,6$ Branched Triantennary Oligosaccharide 11 (400 MHz).

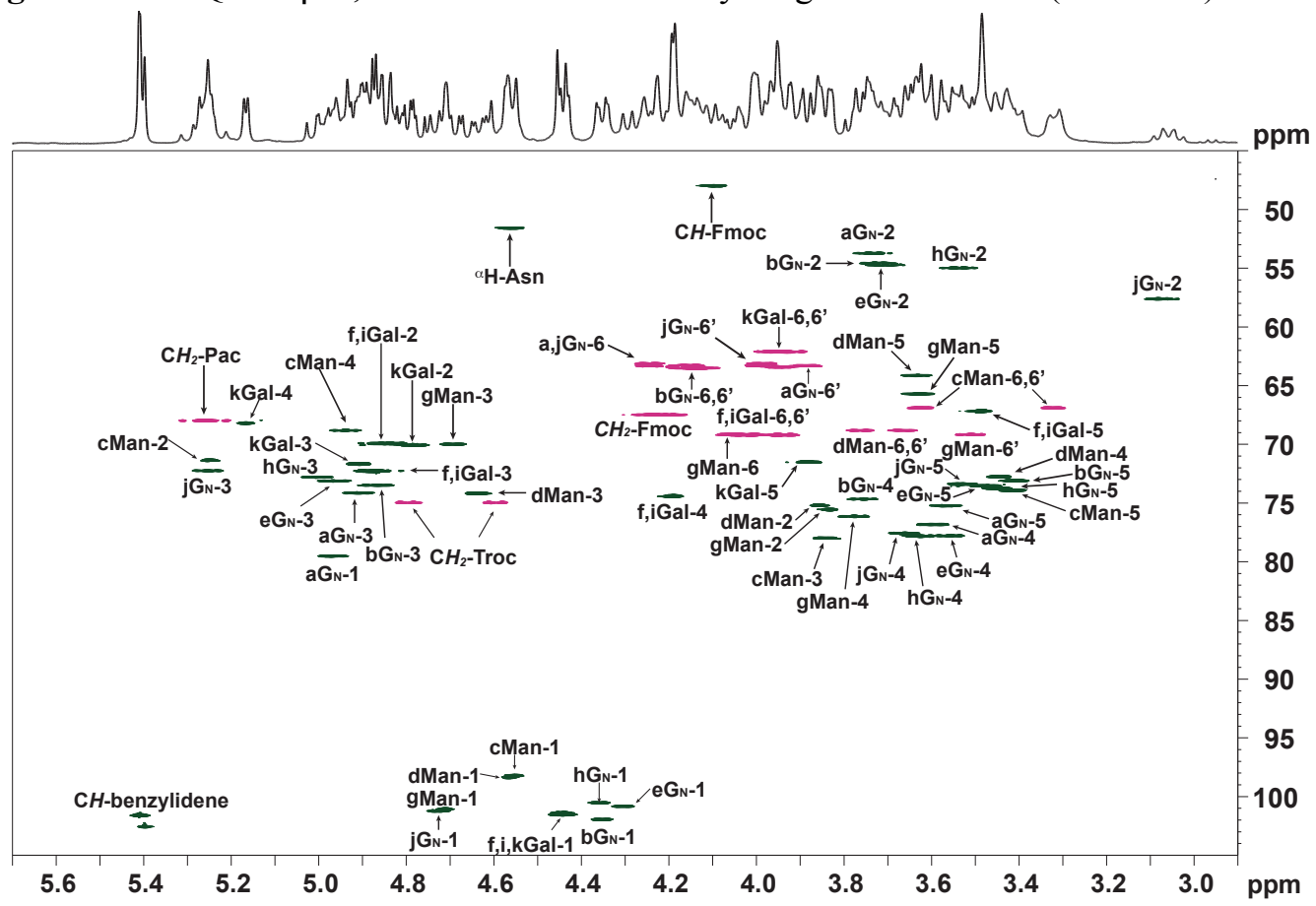

All chemical shifts were assigned by combined 2D NMR experiments. 


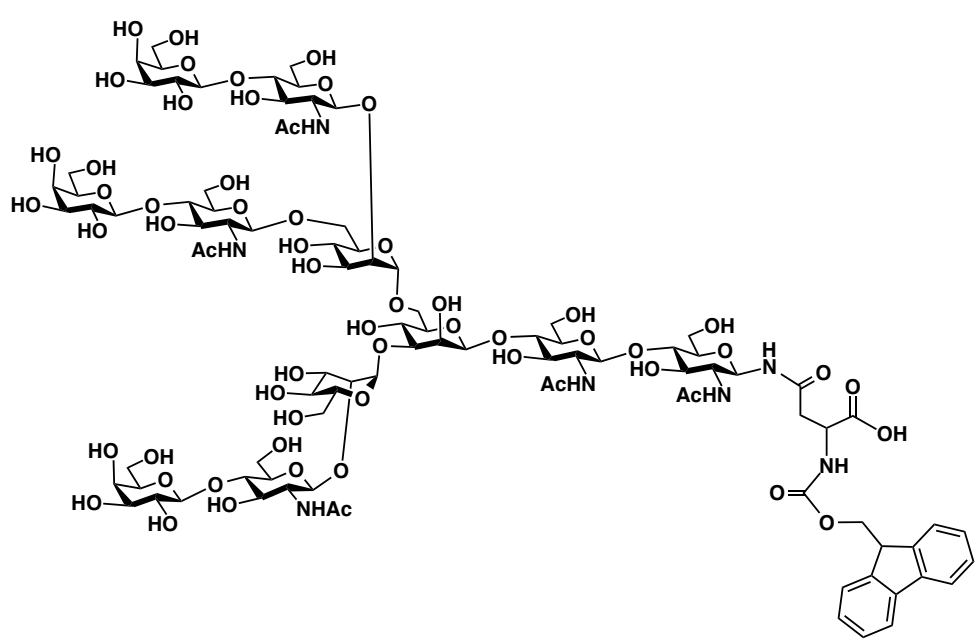

$N^{2}$-(9-Fluorenylmethyloxycarbonyl)- $N^{4}-\{O-\beta$-D-galactopyranosyl-(1 $\rightarrow 4)-O$-(2-acetamido2-deoxy- $\beta$-D-glucopyranosyl)-( $1 \rightarrow 2)-O$ - $\alpha$-D-mannopyranosyl-( $1 \rightarrow 3)-O$-[ $\beta$-Dgalactopyranosyl-( $1 \rightarrow 4)$ - $O$-(2-acetamido-2-deoxy- $\beta$-D-glucopyranosyl)-(1 $\rightarrow 2)-O$-[ $\beta$-Dgalactopyranosyl-(1 $\rightarrow 4)$ - $O$-(2-acetamido-2-deoxy- $\beta$-D-glucopyranosyl)-(1 $\rightarrow 6)]-O$ - $\alpha$-Dmannopyranosyl-(1 $\rightarrow 6)]-O$ - $\beta$-D-mannopyranosyl- $O$ - $(1 \rightarrow 4)$-(2-acetamido-2-deoxy- $\beta$-Dglucopyranosyl)-( $1 \rightarrow 4)$-(2-acetamido-2-deoxy- $\beta$-D-glucopyranosyl)\}-L-asparagine (1).

The protected triantennary oligosaccharide $11(2.3 \mathrm{mg}, 0.61 \mu \mathrm{mol})$ was dried in vacuo for 3 hours, which was then dissolved in THF $(300 \mu \mathrm{L}), \mathrm{AcOH}(200 \mu \mathrm{L})$, and $\mathrm{Ac}_{2} \mathrm{O}(100 \mu \mathrm{L})$ on an ice bath. To the mixture, freshly activated zinc powder $(161 \mathrm{mg}, 2.45 \mathrm{mmol})$ was slowly added. After 1 hour, the resulting mixture was allowed to arise from $0{ }^{\circ} \mathrm{C}$ to $\mathrm{rt}$ and then stirred for additional 15 hours. The reaction was monitored by RP-HPLC and TLC analyses. After completion of the reaction, the mixture was diluted with $\mathrm{CH}_{3} \mathrm{CN}$ and filtered through a pad of Celite, followed by addition of $\mathrm{MeOH}$, the resulting mixture was concentrated to give crude $\mathbf{S 3}$; ESI-MS calcd for $\mathrm{C}_{160} \mathrm{H}_{199} \mathrm{~N}_{7} \mathrm{O}_{82}[\mathrm{M}+2 \mathrm{H}]^{2+}$ 1766.1, found for $m / z$ 1766.2.

To a solution of crude $\mathbf{S 3}$ in ice-cold $\mathrm{MeOH}(256 \mu \mathrm{L})$, ice-cold $5 \mathrm{M}$ aq. $\mathrm{NaOH}(256 \mu \mathrm{L})$ was added slowly, and the mixture was stirred for $30 \mathrm{~min}$ on ice bath and stirred for additional 2 hours at rt. After completion of the reaction, the resulting mixture was subjected directly to gel filtration chromatography $\left(\varphi 1.4 \times 33 \mathrm{~cm}\right.$, Sephadex ${ }^{\mathrm{TM}} \mathrm{G}-15$, eluent: distilled water $)$. Fractions containing oligosaccharide were collected and lyophilized to give crude oligosaccharide S4; ESI-MS calcd for $\mathrm{C}_{101} \mathrm{H}_{145} \mathrm{~N}_{7} \mathrm{O}_{58}[\mathrm{M}+2 \mathrm{H}]^{2+} 1192.9$, found for $m / z$ 1193.0.

To an ice-cold solution of the crude oligosaccharide $\mathbf{S} 4$ in $\mathrm{H}_{2} \mathrm{O}(250 \mathrm{uL})$ containing $\mathrm{NaHCO}_{3}$ (10 umol) were added a solution of FmocOSu $(1.7 \mathrm{mg}, 5.0 \mathrm{umol})$ in acetone $(250 \mathrm{uL})$ slowly. 
The reaction mixture was stirred for $30 \mathrm{~min}$ on an ice bath, which was stirred for additional 3 hours at $\mathrm{rt}$. The resulting mixture was subjected directly to gel filtration chromatography $(\varphi 1.4$ $\times 33 \mathrm{~cm}$, Sephadex ${ }^{\mathrm{TM}} \mathrm{G}-25$, eluent: distilled water). Fractions containing oligosaccharide were collected and lyophilized to give crude S5; ESI-MS calcd for $\mathrm{C}_{116} \mathrm{H}_{155} \mathrm{~N}_{7} \mathrm{O}_{60}[\mathrm{M}+2 \mathrm{H}]^{2+}$ 1304.0, found for $m / z$ 1303.9.

Ice-cold $90 \%$ aq. TFA (255 uL) was slowly added to crude $\mathbf{S 5}$ on ice bath. The mixture was kept at $0{ }^{\circ} \mathrm{C}$ for $10 \mathrm{~min}$, and concentrated at $\mathrm{rt}$, after which distilled water was added. The resulting mixture was frozen by treatment of liquid $\mathrm{N}_{2}$ and lyophilized immediately. The resulting residue was purified by RP-HPLC (Proteonavi $\mathrm{C} 4,0.1 \%$ aq. TFA: $90 \%$ aq. $\mathrm{CH}_{3} \mathrm{CN}$ containing $0.1 \% \mathrm{TFA}=80: 20$ to $50: 50$ over $30 \mathrm{~min}$ at $1 \mathrm{~mL} / \mathrm{min}$ ). Fractions containing the desired product were collected and lyophilized to give Asparagine-linked $\beta$-1,6-branched triantennary asialo-undecasaccharide $1(0.9 \mathrm{mg}, 0.38 \mu \mathrm{mol}, 63 \%)$.

${ }^{1}$ H NMR (400 MHz, D $\mathbf{O}$, 4.79 ppm) $\delta 7.92(\mathrm{~d}, J=7.35 \mathrm{~Hz}, 2 \mathrm{H}), 7.71$ (brd, $\left.J=6.50 \mathrm{~Hz}, 2 \mathrm{H}\right)$, 7.50 (brt, $J=7.34 \mathrm{~Hz}, 2 \mathrm{H}$ ), 7.47-7.40 (m, $2 \mathrm{H}), 5.13$ (s, $1 \mathrm{H}$ ), 5.01 (d, $J=9.55 \mathrm{~Hz}, 1 \mathrm{H}), 4.87$ (s, $1 \mathrm{H}), 4.77$ (s, $1 \mathrm{H}), 4.62-4.57$ (m, $3 \mathrm{H}), 4.56(\mathrm{~d}, \mathrm{~J}=7.77 \mathrm{~Hz}, 1 \mathrm{H}), 4.52-4.42$ (m, $3 \mathrm{H}), 4.39-4.31$ (m, 2 H), 4.25 (brs, $1 \mathrm{H}), 4.24-4.16$ (m, $2 \mathrm{H}), 4.10$ (s, $1 \mathrm{H}), 4.05-3.69$ (m, $45 \mathrm{H}), 3.69-3.65$ (m, 3 H), 3.65-3.45 (m, $13 \mathrm{H}), 3.41(\mathrm{t}, J=9.75 \mathrm{~Hz}, 1 \mathrm{H}), 2.72(\mathrm{dd}, J=15.62,3.96 \mathrm{~Hz}, 1 \mathrm{H}), 2.61(\mathrm{dd}$, $J=15.62,8.07 \mathrm{~Hz}, 1 \mathrm{H}), 2.08$ (s, $3 \mathrm{H}), 2.06$ (s, $3 \mathrm{H}), 2.04$ (s, $6 \mathrm{H}), 1.90$ (s, $3 \mathrm{H}$ ).

Chemical shift of ${ }^{13} \mathrm{C}\left(\mathrm{D}_{2} \mathrm{O}\right.$, chemical shift picked up from HSQC except for quaternary carbon) $\delta 128.96,128.46,126.01,121.08,103.87,102.56,102.29,101.28,100.45,98.13,81.26$, $80.09,79.56,79.45,79.11,77.50,77.33,77.10,76.29,75.65,74.48,73.65,73.45,73.19,72.88$, 72.49, 71.92, 71.28, 71.14, 70.33, 69.47, 68.48, 68.23, 67.76, 67.73, 66.49, 66.10, 62.67, 61.98 . HRMS (ESI): calcd for $\mathrm{C}_{95} \mathrm{H}_{143} \mathrm{~N}_{7} \mathrm{O}_{60}[\mathrm{M}+2 \mathrm{H}]^{2+} 1171.9250$, found for $\mathrm{m} / \mathrm{z} 1171.9246$ $[\mathrm{M}+2 \mathrm{H}]^{2+}$. 


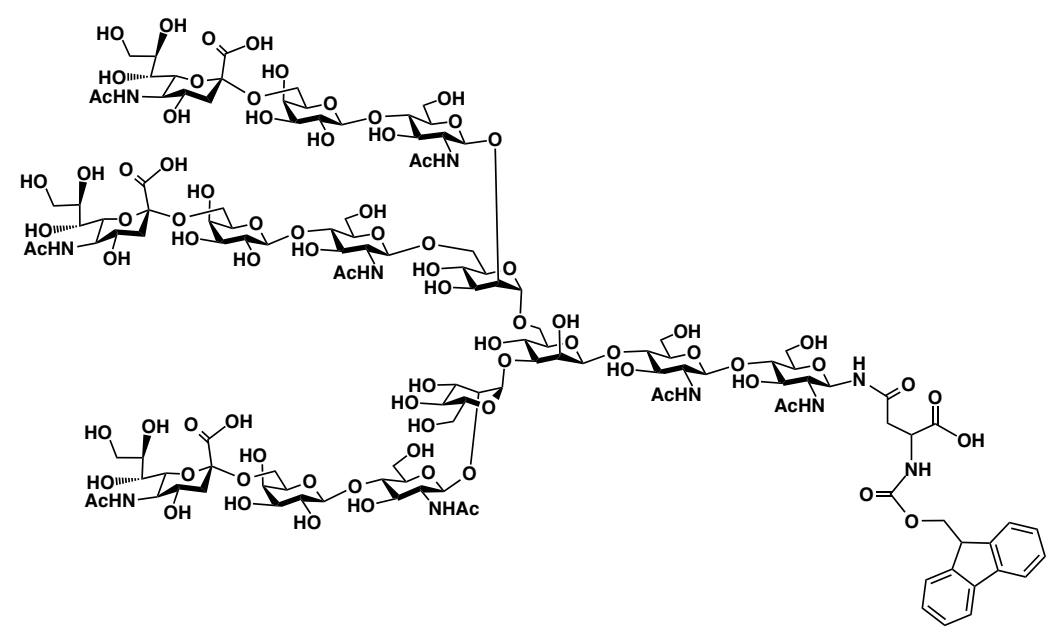

$N^{2}$-(9-Fluorenylmethyloxycarbonyl)- $N^{4}-\{O$-(5-acetamido-3,5-dideoxy-D-glycero- $\alpha-D-$ galacto-2-nonulopyranosylonic $\quad$ acid)-(2 $\rightarrow 6)-O$ - $\beta$-D-galactopyranosyl-(1 $\rightarrow 4)-O-(2-$ acetamido-2-deoxy- $\beta$-D-glucopyranosyl)-(1 $\rightarrow 2)-O$ - $\alpha$-D-mannopyranosyl-(1 $\rightarrow 3)$-O-[(5acetamido-3,5-dideoxy-D-glycero- $\alpha$-D-galacto-2-nonulopyranosylonic $\quad$ acid)-(2 $\rightarrow 6)-\beta$-Dgalactopyranosyl-( $\rightarrow 4)$ - $O$-(2-acetamido-2-deoxy- $\beta$-D-glucopyranosyl)-(1 $\rightarrow 2)-O$-[(5acetamido-3,5-dideoxy-D-glycero- $\alpha$-D-galacto-2-nonulopyranosylonic $\quad$ acid)-(2 $\rightarrow 6)-\beta$-Dgalactopyranosyl-(1 $\rightarrow 4)$ - $O$-(2-acetamido-2-deoxy- $\beta$-D-glucopyranosyl)-(1 $\rightarrow 6)]-O-\alpha$-D-

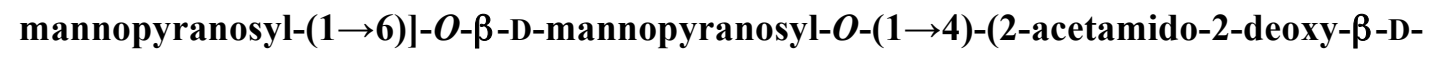
glucopyranosyl)-(1 $\rightarrow 4)$-(2-acetamido-2-deoxy- $\beta$-D-glucopyranosyl)\}-L-asparagine (13).

Asialo-oligosaccharide $1(0.13 \mu \mathrm{mol})$ and CMP-Neu5Ac (1.2 $\mu \mathrm{mol}, 9.1$ equiv) were dissolved in $100 \mathrm{mM}$ 4-(2-hydroxyethyl)-1-piperazineethanesulfonic acid (HEPES) buffer (pH 6.1) containing BSA $(0.1 \mathrm{mg} / \mathrm{mL})$ and alkaline phosphatase $(100 \mathrm{mU})$. To the reaction mixture, $\alpha$-2,6-sialyltransferase ( $3 \mathrm{U}$ ) was added (total volume $=65 \mathrm{uL}$ ), and the reaction mixture was incubated at rt. The reaction was monitored with LC/MS. After 48 hours, Additional CMP-Neu5Ac (1.6 $\mu \mathrm{mol})$, Alkaline phosphatase (100 mU) and $\alpha$-2,6-sialyltransferase (1.5 U) were added and then incubated for another 2 days at $\mathrm{rt}$. The resulting mixture was filtered, and the filtrate was purified by RP-HPLC (Proteonavi C4, $50 \mathrm{mM}$ aq. $\mathrm{MH}_{4} \mathrm{OAc}$ : $\mathrm{CH}_{3} \mathrm{CN}=90: 10$ to 80: 20 over $30 \mathrm{~min}$ at $1 \mathrm{~mL} / \mathrm{min}$ ). Fractions containing desired product were collected and lyophilized, which was further purified by RP-HPLC (Proteonavi C4, distilled water over 30 $\mathrm{min}$ at $1 \mathrm{~mL} / \mathrm{min}$ ). Fractions containing desired product were collected and lyophilized to provide tri-sialylated oligosaccharide 13 (isolated; ca. $30 \mathrm{nmol}$, conversion yield; $86 \%$ based on 
HPLC analysis).

${ }^{1} \mathrm{H}$ NMR $\left(\mathrm{D}_{2} \mathrm{O}\right) \delta 7.83(\mathrm{~d}, J=7.68 \mathrm{~Hz}, 2 \mathrm{H}), \delta 7.92(\mathrm{~d}, J=7.35 \mathrm{~Hz}, 2 \mathrm{H}), 7.65-7.61(\mathrm{~m}, 2 \mathrm{H})$, 7.44-7.40 (m, 2 H), 7.37-7.32 (m, 2 H), 5.04 (s, 1 H), 4.38-4.33 (m), 4.29-4.24 (m), 4.20-4.15 (m), 4.15-4.10 (m), 4.03-3.99 (m), 3.95-3.38 (m), 2.65-2.55 (m, 4 H), 2.47 (m, 1 H), 1.98 (s, 9 H), 1.94 (s, 9 H), 1.82 (s, 3 H), 1.80 (s, 3 H), 1.67-1.60 (m, 3 H).

HRMS (ESI): calcd for $\mathrm{C}_{128} \mathrm{H}_{194} \mathrm{~N}_{10} \mathrm{O}_{84}[\mathrm{M}-3 \mathrm{H}]^{+} 1070.6989$, found for $m / z 1070.7012[\mathrm{M}-3 \mathrm{H}]^{3-}$. 


\subsection{Synthesis of a Triantennary Oligosaccharide Having $\beta-1,4-B r a n c h i n g$}

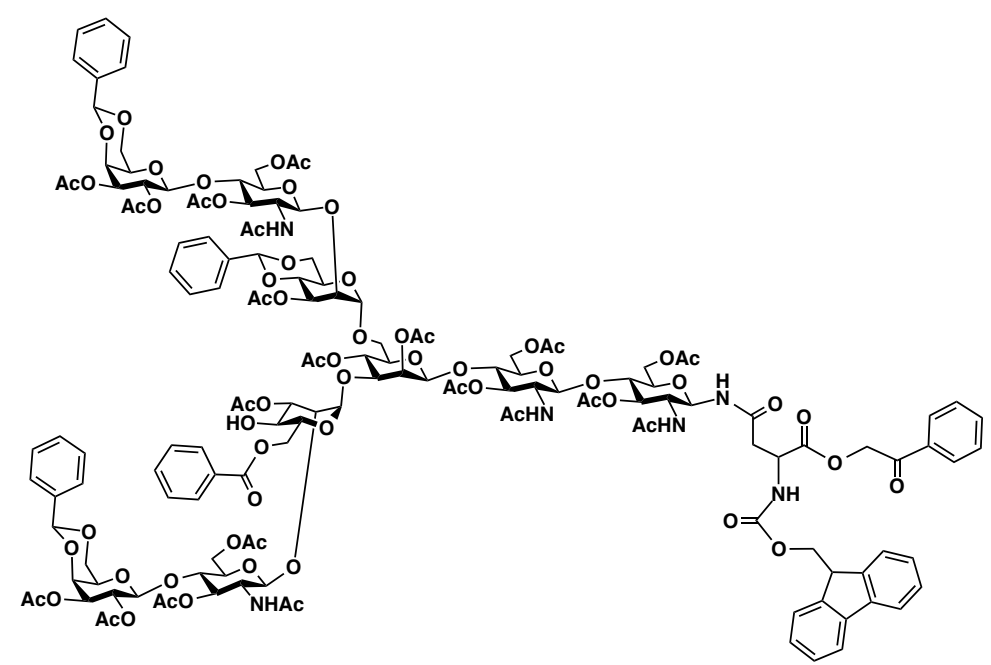

$N^{2}$-(9-Fluorenylmethyloxycarbonyl)- $N^{4}-\{O$-(2,3-di- $O$-acetyl-4,6- $O$-benzylidene- $\beta$-Dgalactopyranosyl)-(1 $\rightarrow 4)$ - $O$-(2-acetamido-3,6-di- $O$-acetyl-2-deoxy- $\beta$-D-glucopyranosyl)-(1 $\rightarrow 2)$ - $O$-(3-O-acetyl-6- $O$-benzoyl- $\alpha$-D-mannopyranosyl)-(1 $\rightarrow 3)$ - $O$-[(2,3-di- $O$-acetyl-4,6-O-be nzylidene- $\beta$-D-galactopyranosyl)-(1 $\rightarrow 4)$ - $O$-(2-acetamido-3,6-di- $O$-acetyl-2-deoxy- $\beta$-D-gluc opyranosyl)-(1 $\rightarrow 2)$ - $O$-(3- $O$-acetyl-4,6- $O$-benzylidene- $\alpha$-D-mannopyranosyl)-(1 $\rightarrow 6)]-O$ - $(2,4$ -di- $O$-acetyl- $\beta$-D-mannopyranosyl)-(1 $\rightarrow 4)$ - $O$-(2-acetamido-3,6-di- $O$-acetyl-2-deoxy- $\beta$-D-glu copyranosyl)-(1 $\rightarrow 4)$-(2-acetamido-3,6-di- $O$-acetyl-2-deoxy- $\beta$-D-glucopyranosyl) $\}$-Lasparagine phenacyl ester (9).

The tri-benzylidene $7(11.8 \mathrm{mg}, 3.9 \mu \mathrm{mol})$ was dried in vacuo for 2 hours, which was then dissolved in $\mathrm{CH}_{2} \mathrm{Cl}_{2}(780 \mathrm{uL})$, followed by addition of $N, N^{\prime}$-diisopropylethylamine (10 $\mu \mathrm{L}, 59$ $\mu \mathrm{mol})$ and $\mathrm{BzCN}(4.6 \mu \mathrm{L}, 38.8 \mu \mathrm{mol})$, the resulting mixture was stirred at $\mathrm{rt}$ for 2 hours. The reaction was monitored by HPLC and TLC analyses. After a completion of the reaction, the mixture was diluted with chloroform and directly purified by silica gel chromatography to remove reagents $(\varphi 1.0 \times 8.0 \mathrm{~cm}$, EtOAc, EtOAc: $\mathrm{MeOH}(5: 1))$. The fractions containing oligosaccharides were collected and then evaporated. The resulting residue was dissolved in aq. $\mathrm{CH}_{3} \mathrm{CN}$, filtered, and purified by preparative RP-HPLC (XBridge, $50 \mathrm{mM}$ aq. $\mathrm{NH}_{4} \mathrm{Ac}$ : $\mathrm{CH}_{3} \mathrm{CN}$ $=45: 55$ to $35: 65$ over $90 \mathrm{~min}$ at $4 \mathrm{~mL} / \mathrm{min}$ ). Fractions containing the desired product was collected, treated with Dowex, and lyophilized to give glycosyl acceptor 9 (5.8 mg, $1.9 \mu \mathrm{mol}$, $48 \%)$.

${ }^{1}$ H NMR (400 MHz, CD 3 CN, HOD 2.13 ppm) $\delta 7.79(\mathrm{~d}, J=7.36 \mathrm{~Hz}, 2 \mathrm{H}), 7.66$ (d, $J=7.72$ $\mathrm{Hz}, 2 \mathrm{H}), 7.55$ (d, $J=7.59 \mathrm{~Hz}, 2 \mathrm{H}), 7.42-7.35$ (m, 4 H), 7.33-7.22 (m, 4 H), 7.22-7.01 (m, 19 
H), $6.36(\mathrm{~d}, J=9.73 \mathrm{~Hz}, 1 \mathrm{H}), 6.29(\mathrm{~d}, J=9.20 \mathrm{~Hz}, 1 \mathrm{H}), 6.25(\mathrm{~d}, J=9.51 \mathrm{~Hz}), 6.14(\mathrm{~d}, J=9.28$ $\mathrm{Hz}, 1 \mathrm{H}), 6.08$ (d, $J=8.82 \mathrm{~Hz}, 1 \mathrm{H}), 5.30(\mathrm{~s}, 1 \mathrm{H}), 5.27$ (s, $2 \mathrm{H}), 5.20-5.06$ (m, $3 \mathrm{H}), 4.99$ (dd, $J$ = 9.91, $9.84 \mathrm{~Hz}, 1 \mathrm{H}), 4.89-4.66(\mathrm{~m}, 10 \mathrm{H}), 4.60(\mathrm{~s}, 1 \mathrm{H}), 4.50(\mathrm{~s}, 1 \mathrm{H}), 4.47-4.36(\mathrm{~m}, 3 \mathrm{H})$, 4.35-4.26 (m, $3 \mathrm{H}), 4.24-3.77$ (m, $22 \mathrm{H})$, 3.77-3.70 (m, $2 \mathrm{H}), 3.67-3.20$ (m, $23 \mathrm{H}), 2.57-2.42$ (m, 2 H), 1.88 (s, 3 H), 1.82 (s, 3 H), 1.81 (s, 3 H), 1.78 (s, 9 H), 1.75 (s, 6 H), 1.74 (s, 3 H), 1.73 (s, $3 \mathrm{H}), 1.72(\mathrm{~s}, 3 \mathrm{H}), 1.68(\mathrm{~s}, 12 \mathrm{H}), 1.61(\mathrm{~s}, 3 \mathrm{H}), 1.58(\mathrm{~s}, 3 \mathrm{H}), 1.55(\mathrm{~s}, 3 \mathrm{H}), 1.48(\mathrm{~s}, 3 \mathrm{H}), 1.39$ (s, $3 \mathrm{H})$.

${ }^{13}$ C NMR (100 MHz, CD ${ }_{3}$ CN, 118.26 ppm) $8193.39,172.07,171.91,171.74,171.66,171.57$, $171.49,171.41,171.33$, 171.27, 171.23, 171.20, 171.15, 171.11, 170.98, 170.67, 170.63, 170.57, $167.19,159.73,145.06,142.14,139.33,139.30,38.79$, 135.07, 135.04, 134.24, 131.08, 130.58, $130.10,129.92$, 129.85, 129.26, 129.24, 129.19, 128.80, 128.73, 128.17, 127.47, 127.31, 126.20 , $121.01,102.79,101.68,101.61,101.18,101.05,100.77,99.65,99.04,79.55,78.20,77.90$, $77.72,76.78,76.41,76.02,75.57,75.51,75.31,74.48,74.43,73.99,73.79,73.69,73.31,73.06$, $72.36,72.27,71.71,70.41,69.97,69.92,69.38,69.20,68.56,68.00,67.47,67.23,67.15,64.92$, $64.35,63.36,55.03,54.80,54.21,53.83,51.57,47.98,38.31,23.27,23.23,23.09,21.68,21.57$, $21.25,21.20,21.14,21.07,21.02,20.97,20.94$.

HRMS (ESI): calcd for $\mathrm{C}_{149} \mathrm{H}_{174} \mathrm{~N}_{6} \mathrm{O}_{68}[\mathrm{M}+2 \mathrm{H}]^{2+} 1568.5244$, found for $m / z 1568.5292$ $[\mathrm{M}+2 \mathrm{H}]^{2+}$.

Assignment: HSQC of Benzoate 9 (400 MHz).

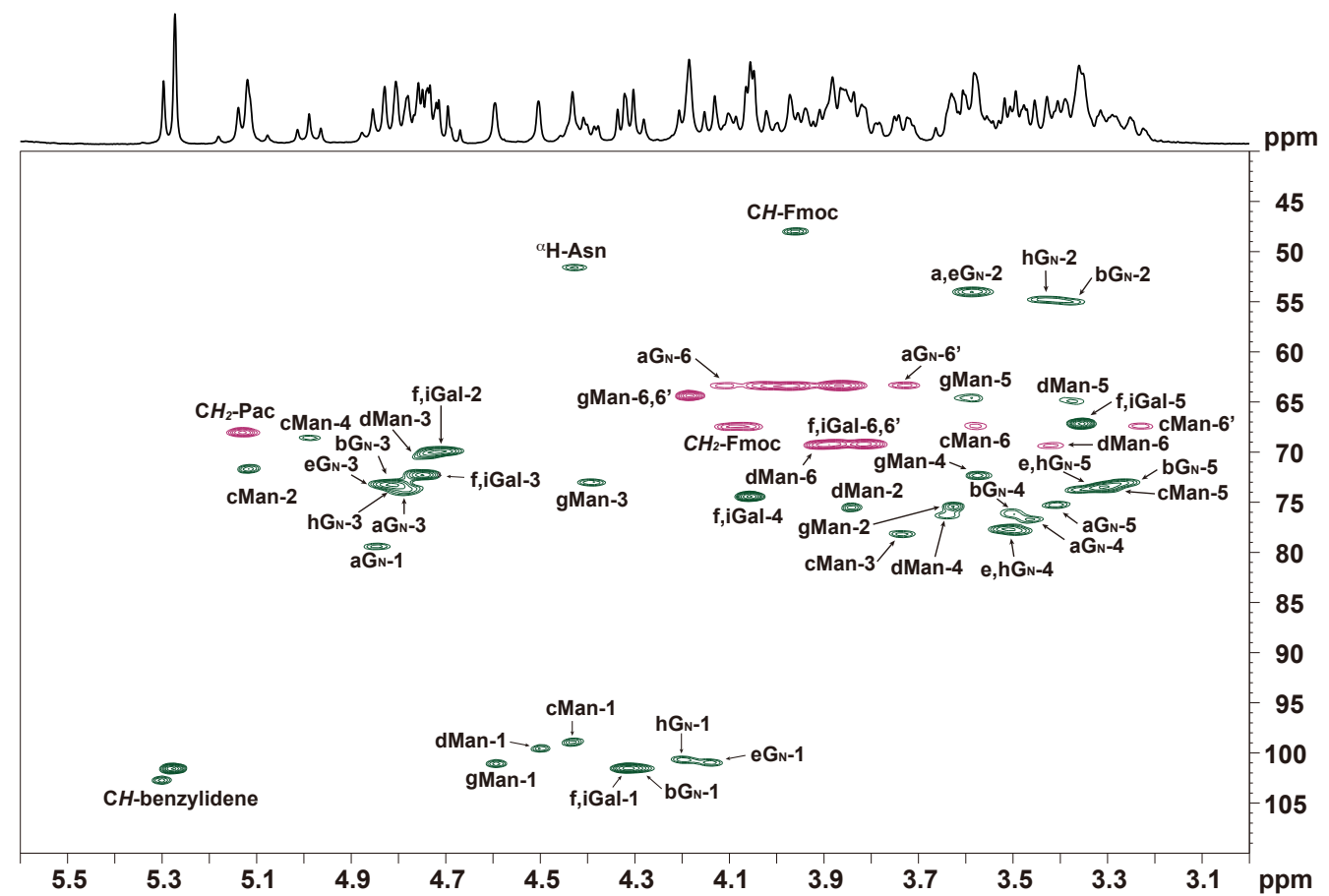

All chemical shifts were assigned by combined 2D NMR experiments. 


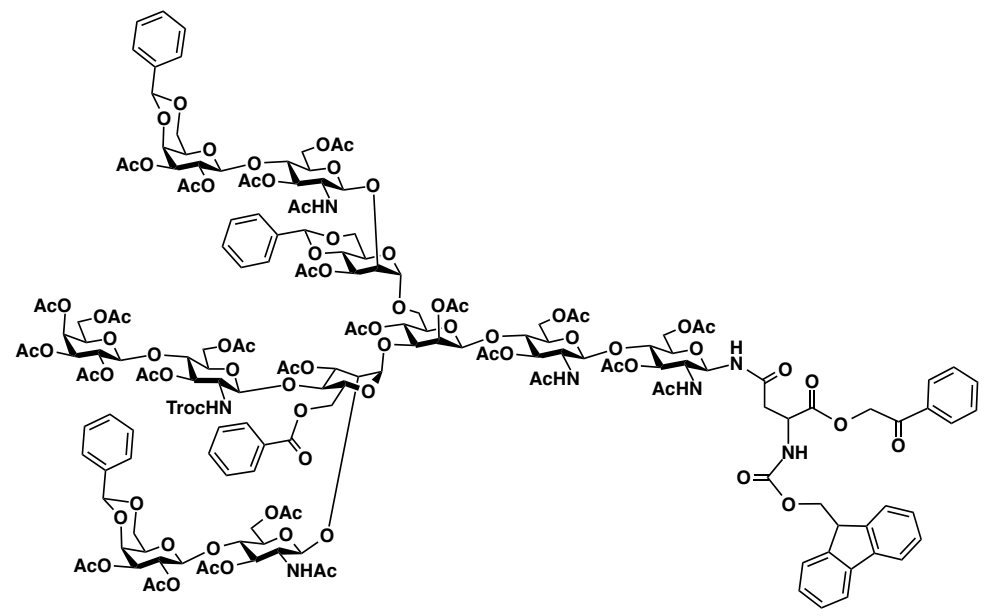

$N^{2}$-(9-Fluorenylmethyloxycarbonyl)- $N^{4}-\{O$-(2,3-di- $O$-acetyl-4,6-O-benzylidene- $\beta$-Dgalactopyranosyl)-(1 $\rightarrow 4)$ - $O$-(2-acetamido-3,6-di- $O$-acetyl-2-deoxy- $\beta$-D-glucopyranosyl)$(1 \rightarrow 2)$ - $O$-[ $(2,3,4,6$-tetra- $O$-acetyl- $\beta$-D-galactopyranosyl)-(1 $\rightarrow 4)$ - $O$-(3,6-di- $O$-acetyl-2-deoxy2-(2,2,2-trichloroethoxy)-carbonylamino- $\beta$-D-glucopyranosyl)-(1 $\rightarrow 4)]-O$-(3-O-acetyl-6-Obenzoyl- $\alpha$-D-mannopyranosyl)-( $1 \rightarrow 3)-O$-[(2,3-di- $O$-acetyl-4,6- $O$-benzylidene- $\beta$-D-galactopyranosyl)-( $1 \rightarrow 4)$ - $O$-(2-acetamido-3,6-di- $O$-acetyl-2-deoxy- $\beta$-D-glucopyranosyl)-(1 $\rightarrow 2)-O$ (3-O-acetyl-4,6-O-benzylidene- $\alpha$-D-mannopyranosyl)-( $1 \rightarrow 6)]-O$-(2,4-di- $O$-acetyl- $\beta$-Dmannopyranosyl)-(1 $\rightarrow 4)$ - $O$-(2-acetamido-3,6-di- $O$-acetyl-2-deoxy- $\beta$-D-glucopyranosyl)$(1 \rightarrow 4)$-(2-acetamido-3,6-di- $O$-acetyl-2-deoxy- $\beta$-D-glucopyranosyl)\}-L-asparagine phenacyl ester (12).

A mixture of glycosyl acceptor $\mathbf{9}(5.3 \mathrm{mg}, 1.7 \mu \mathrm{mol})$ and glycosyl donor $\mathbf{1 0}(29.1 \mathrm{mg}, 33.8$ $\mu \mathrm{mol})$ was in vacuo for 3 hours, after which this mixture was dissolved in distilled $\mathrm{CH}_{2} \mathrm{Cl}_{2}(320$ $\mu \mathrm{L}$ ) under an atmosphere of Ar, followed by addition of freshly activated 4 A molecular sieves $(35 \mathrm{mg})$. The reaction mixture was allowed to cool to $0{ }^{\circ} \mathrm{C}$, followed by addition of NIS $(6.1 \mathrm{mg}$, $27.1 \mu \mathrm{mol})$. Then, TfOH $\left(1.27 \mu \mathrm{mol}\right.$, dilution with $\left.\mathrm{CH}_{2} \mathrm{Cl}_{2}\right)$ was added to the mixture and stirred for 1.5 hours at the same temperature, after which the temperature was elevated to $\mathrm{rt}$ and stirred for additional $30 \mathrm{~min}$. The resulting mixture was directly applied on silica gel column chromatography to remove glycosyl donor derivatives $(\phi 1.0 \times 7.0 \mathrm{~cm}$, Hexane: EtOAc (1: 1), EtOAc, EtOAc: $\mathrm{MeOH}(5: 1))$. The fractions containing oligosaccharides were collected and then evaporated. The resulting residue was dissolved in $\mathrm{CH}_{3} \mathrm{CN}-\mathrm{H}_{2} \mathrm{O}$, filtered, and purified by preparative RP-HPLC (XBridge, $50 \mathrm{mM}$ aq. $\mathrm{NH}_{4} \mathrm{OAc}: \mathrm{CH}_{3} \mathrm{CN}=45: 55$ to 35: 65 over $90 \mathrm{~min}$ at $4 \mathrm{~mL} / \mathrm{min}$ ). Fractions containing the desired product were collected, treated with Dowex, filtered, and lyophilized to yield protected triantennary oligosaccharide $12(2.9 \mathrm{mg}, 0.75 \mu \mathrm{mol}$, 
$44 \%)$ as a white form.

${ }^{1}$ H NMR (400 MHz, CD 3 CN, HOD 2.13 ppm) $\delta 7.98(\mathrm{~d}, J=7.46 \mathrm{~Hz}, 2 \mathrm{H}), 7.86(\mathrm{~d}, J=7.52$ Hz, 2 H), 7.75 (d, $J=7.51$ Hz, 2 H), 7.61-7.55 (m, 4 H), 7.52-7.42 (m, 4 H), 7.42-7.20 (m, 19 H), $6.45(\mathrm{~d}, J=9.86 \mathrm{~Hz}, 1 \mathrm{H}), 6.39(\mathrm{~d}, J=9.40 \mathrm{~Hz}, 1 \mathrm{H}), 6.36(\mathrm{~d}, J=9.70 \mathrm{~Hz}, 1 \mathrm{H}), 6.21(\mathrm{~d}, J=$ $8.71 \mathrm{~Hz}, 1 \mathrm{H}), 6.11(\mathrm{~d}, J=9.24 \mathrm{~Hz}, 1 \mathrm{H}), 6.03(\mathrm{~d}, J=9.79 \mathrm{~Hz}, 1 \mathrm{H}), 5.49(\mathrm{~s}, 1 \mathrm{H}), 5.47(\mathrm{~s}, 2 \mathrm{H})$, 5.39-5.25 (m, $3 \mathrm{H}), 5.23-5.14$ (m, $2 \mathrm{H}), 5.08-4.85$ (m, $12 \mathrm{H}), 4.84-4.71$ (m, $4 \mathrm{H}), 4.69$ (s, $1 \mathrm{H})$, 4.66-4.59 (m, $3 \mathrm{H}), 4.57-4.44$ (m, 5 H), 4.39 (d, $J=8.40 \mathrm{~Hz}, 1 \mathrm{H}), 4.36-3.87$ (m, $28 \mathrm{H})$, 3.87-3.63 (m, $11 \mathrm{H}), 3.63-3.33$ (m, 14 H), 2.76-2.60 (m, 2 H), 2.06 (s, 3 H), 2.03 (s, 3 H), 2.01 (s, 6 H), 1.99 (s, 6 H), 1.98 (s, 6 H), 1.93 (s, 15 H), 1.92 (s, 3 H), 1.91 (s, 3 H), 1.90 (s, 3 H), 1.89 (s, 12 H), 1.88 (s, $3 \mathrm{H}), 1.85$ (s, $3 \mathrm{H}), 1.81$ (s, $3 \mathrm{H}), 1.76$ (s, $3 \mathrm{H}), 1.74$ (s, $3 \mathrm{H}), 1.68$ (s, 3 $\mathrm{H})$.

Chemical shift of ${ }^{13} \mathrm{C}\left(\mathrm{CD}_{3} \mathrm{CN}\right.$, chemical shift was picked up from $\mathrm{HSQC}$ except for quaternary carbon) $\delta 134.53,130.56,129.92,129.59,128.88,128.65,127.97,127.21,126.03$, $120.86,102.61,101.50,101.43,101.34,100.89$, 100.63, 100.45, 99.53, 98.85, 79.37, 78.13, $77.58,76.92,76.60,76.25,75.90,75.42,75.25,75.18,74.74,74.31,73.87,73.65,73.58,73.52$, 73.44, 73.10, 72.92, 72.14, 71.51, 71.40, 70.26, 69.89, 69.82, 69.19, 69.08, 68.48, 68.04, 67.86, $67.37,67.04,64.82,63.78,63.22,63.17,61.88,57.33,54.83,54.12,54.07,53.70,51.45,47.85$, $38.18,23.03,21.53,21.01,20.63$.

HRMS (ESI): calcd for $\mathrm{C}_{176} \mathrm{H}_{208} \mathrm{Cl}_{3} \mathrm{~N}_{7} \mathrm{O}_{85}[\mathrm{M}+2 \mathrm{H}]^{2+} 1943.0690$, found for $\mathrm{m} / z 1943.0666$ $[\mathrm{M}+2 \mathrm{H}]^{2+}$. 


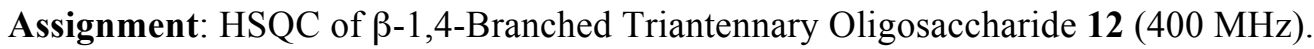

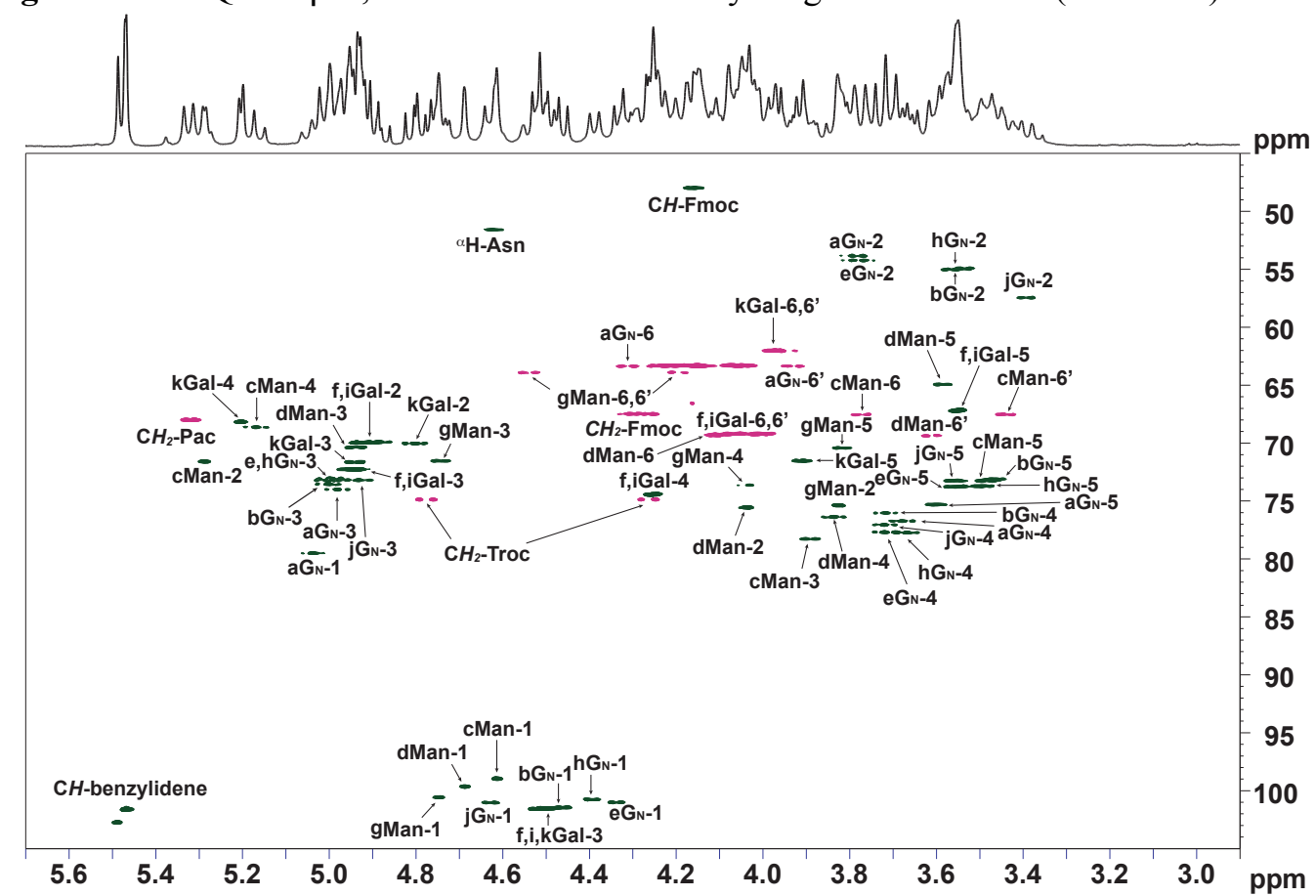

All chemical shifts were assigned by combined 2D NMR experiments. 


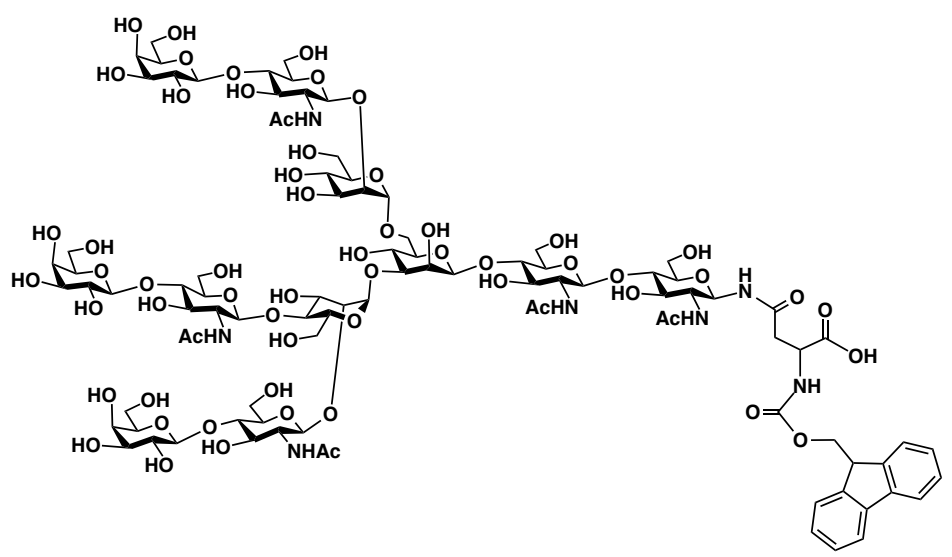

$N^{2}$-(9-Fluorenylmethyloxycarbonyl)- $N^{4}$ - $\{O$ - $\beta$-D-galactopyranosyl-(1 $\rightarrow 4)-O$-(2-acetamido-2 -deoxy- $\beta$-D-glucopyranosyl)-( $1 \rightarrow 2)-O$-[ $\beta$-D-galactopyranosyl-( $1 \rightarrow 4)$ - $O$-(2-acetamido-2deoxy- $\beta$-D-glucopyranosyl)-(1 $\rightarrow 4)]-O$ - $\alpha$-D-mannopyranosyl- $(1 \rightarrow 3)-O$-[ $\beta$-Dgalactopyranosyl-( $1 \rightarrow 4)$ - $O$-(2-acetamido-3,6-di- $O$-acetyl-2-deoxy- $\beta$-D-glucopyranosyl)$(1 \rightarrow 2)-O$ - $\alpha$-D-mannopyranosyl-( $1 \rightarrow 6)]-O$ - $\beta$-D-mannopyranosyl-( $\rightarrow 4)$ - $O$-(2-acetamido-3,6 -di- $O$-acetyl-2-deoxy- $\beta$-D-glucopyranosyl)-(1 $\rightarrow 4)$-(2-acetamido-3,6-di- $O$-acetyl-2-deoxy- $\beta$ D-glucopyranosyl)\}-L-asparagine (2).

The protected triantennary oligosaccharide $12(3.0 \mathrm{mg}, 0.77 \mu \mathrm{mol})$ was dried in vacuo for 2 hours, which was dissolved in THF $(384 \mu \mathrm{L}), \mathrm{AcOH}(256 \mu \mathrm{L})$, and $\mathrm{Ac}_{2} \mathrm{O}(128 \mu \mathrm{L})$ on an ice bath. To the mixture, freshly activated zinc powder $(200 \mathrm{mg}, 3.06 \mathrm{mmol})$ was slowly added. The resulting mixture was stirred at $0{ }^{\circ} \mathrm{C}$ for 1 hour and stirred at $\mathrm{rt}$ for additional 17 hours. The reaction was monitored by RP-HPLC and TLC analyses. After completion of the reaction, the mixture was diluted with $\mathrm{CH}_{3} \mathrm{CN}$ and filtered through a pad of celite, followed by addition of $\mathrm{MeOH}$, the resulting mixture was concentrated to give crude S6; ESI-MS calcd for $\mathrm{C}_{167} \mathrm{H}_{203} \mathrm{~N}_{7} \mathrm{O}_{83}[\mathrm{M}+2 \mathrm{H}]^{2+}$ 1818.1, found for $m / z$ 1818.1.

To a solution of crude $\mathbf{S 6}$ in ice-cold $\mathrm{MeOH}(385 \mu \mathrm{L})$, ice-cold $5 \mathrm{M}$ aq. $\mathrm{NaOH}(385 \mu \mathrm{L})$ was added slowly, and the mixture was stirred for $30 \mathrm{~min}$ on an ice bath, after which the reaction temperature was allowed to warm up to rt and stirred for additional $90 \mathrm{~min}$. After completion of the reaction, the resulting mixture was directly subjected to gel filtration chromatography $(\varphi 1.4$ $\times 33 \mathrm{~cm}$, Sephadex ${ }^{\mathrm{TM}} \mathrm{G}-15$, eluent: distilled water). Fractions containing oligosaccharide were collected and lyophilized to give crude oligosaccharide S7; ESI-MS calcd for $\mathrm{C}_{103} \mathrm{H}_{147} \mathrm{~N}_{7} \mathrm{O}_{59}$ $[\mathrm{M}+2 \mathrm{H}]^{2+} 1192.9$, found for $m / z 1192.9$.

To an ice-cold solution of the crude oligosaccharide $\mathbf{S} 7$ in $\mathrm{H}_{2} \mathrm{O}(385 \mu \mathrm{L})$ containing $\mathrm{NaHCO}_{3}$ 
(16 $\mu \mathrm{mol})$ were added a solution of FmocOSu $(2.6 \mathrm{mg}, 7.7 \mu \mathrm{mol})$ in acetone $(385 \mu \mathrm{L})$. The reaction mixture was stirred for $30 \mathrm{~min}$ on ice bath, after which the reaction mixture was allowed to warm up to $\mathrm{rt}$ and stirred at the same temperature for additional 2.5 hours. The resulting mixture was subjected directly to gel filtration chromatography $(\varphi 1.4 \times 33 \mathrm{~cm}$, Sephadex ${ }^{\mathrm{TM}} \mathrm{G}-25$, eluent: distilled water). Fractions containing oligosaccharide were collected and lyophilized to give crude S8; ESI-MS calcd for $\mathrm{C}_{116} \mathrm{H}_{155} \mathrm{~N}_{7} \mathrm{O}_{60}[\mathrm{M}+2 \mathrm{H}]^{2+}$ 1304.0, found for $m / z 1304.0$.

Ice-cold $90 \%$ aq. TFA (385 $\mu \mathrm{L})$ was slowly added to crude $\mathbf{S 8}$ on an ice bath. The mixture was kept at $0{ }^{\circ} \mathrm{C}$ for $10 \mathrm{~min}$, and concentrated at $\mathrm{rt}$, after which distilled water was added. The solution was immediately frozen by the treatment of liquid $\mathrm{N}_{2}$ and then lyophilized. The resulting residue was purified with RP-HPLC (Proteonavi $\mathrm{C} 4,0.1 \%$ aq. TFA: $90 \%$ aq. $\mathrm{CH}_{3} \mathrm{CN}$ containing $0.1 \% \mathrm{TFA}=80: 20$ to $50: 50$ over $30 \mathrm{~min}$ at $1 \mathrm{~mL} / \mathrm{min}$ ). Fractions containing the desired product were collected and lyophilized to give Asn-linked triantennary asialo-undecasaccharide 2 ( $0.8 \mathrm{mg}, 0.34 \mu \mathrm{mol}, 44 \%)$.

${ }^{1}$ H NMR (700 MHz, D $\mathbf{2}$, 4.76 ppm) $\delta 7.92(\mathrm{~d}, J=7.34 \mathrm{~Hz}, 2 \mathrm{H}), 7.71(\mathrm{~d}, J=6.11 \mathrm{~Hz}, 2 \mathrm{H})$, $7.51(\mathrm{t}, J=7.33 \mathrm{~Hz}, 2 \mathrm{H}), 7.47-7.37(\mathrm{~m}, 2 \mathrm{H}), 5.12(\mathrm{~s}, 1 \mathrm{H}), 5.01$ (d, $J=9.23 \mathrm{~Hz}, 1 \mathrm{H}), 4.92(\mathrm{~s}, 1$ H), 4.75 (s, $1 \mathrm{H}), 4.64-4.50(\mathrm{~m}, 6 \mathrm{H}), 4.50-4.43$ (m, $3 \mathrm{H}), 4.39-4.28$ (m, $2 \mathrm{H}), 4.22$ (brs, $2 \mathrm{H})$, 4.11 (brs, $1 \mathrm{H}), 4.05$ (d, $J=9.28 \mathrm{~Hz}, 1 \mathrm{H}), 4.03-3.94(\mathrm{~m}, 4 \mathrm{H}), 3.94-3.65(\mathrm{~m}, 42 \mathrm{H}), 3.65-3.51$ (m, $14 \mathrm{H}), 3.51-3.43$ (m, $2 \mathrm{H}), 2.77-2.67$ (dd, $J=15.40,2.66 \mathrm{~Hz}, 1 \mathrm{H}), 2.63-2.52$ (dd, $J=15.47$, $8.73 \mathrm{~Hz}, 1 \mathrm{H}), 2.11-2.06$ (s, $6 \mathrm{H}), 2.06-2.01(\mathrm{~s}, 6 \mathrm{H}), 1.93-1.86(\mathrm{~s}, 3 \mathrm{H})$.

\section{Chemical shift of ${ }^{13} \mathrm{C}\left(\mathrm{D}_{2} \mathrm{O}\right.$, chemical shift was picked up from HSQC except for} quaternary carbon) $\delta 128.97,128.45,126.02,121.08,103.88,102.52,120.23,101.36,100.45$, $100.08,98.00,81.33,80.33,79.55,79.46,79.11,79.04,77.26,77.13,76.94,76.31,75.75,75.65$, $75.33,73.79,73.68,73.44,73.04,72.91,71.93,71.10,70.38,69.48,69.02,69.27,67.35,66.68$, $62.59,62.19,61.95,60.87,60.70,56.09,55.81,54.72,53.02,47.92,39.12,23.47,23.29,22.96$.

HRMS (ESI): calcd for $\mathrm{C}_{95} \mathrm{H}_{143} \mathrm{~N}_{7} \mathrm{O}_{60}[\mathrm{M}+2 \mathrm{H}]^{2+} 1171.9250$, found for $\mathrm{m} / \mathrm{z} 1171.9263$ $[\mathrm{M}+2 \mathrm{H}]^{2+}$. 


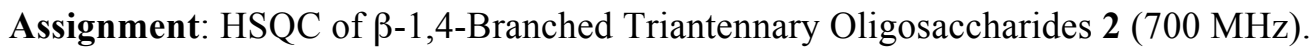

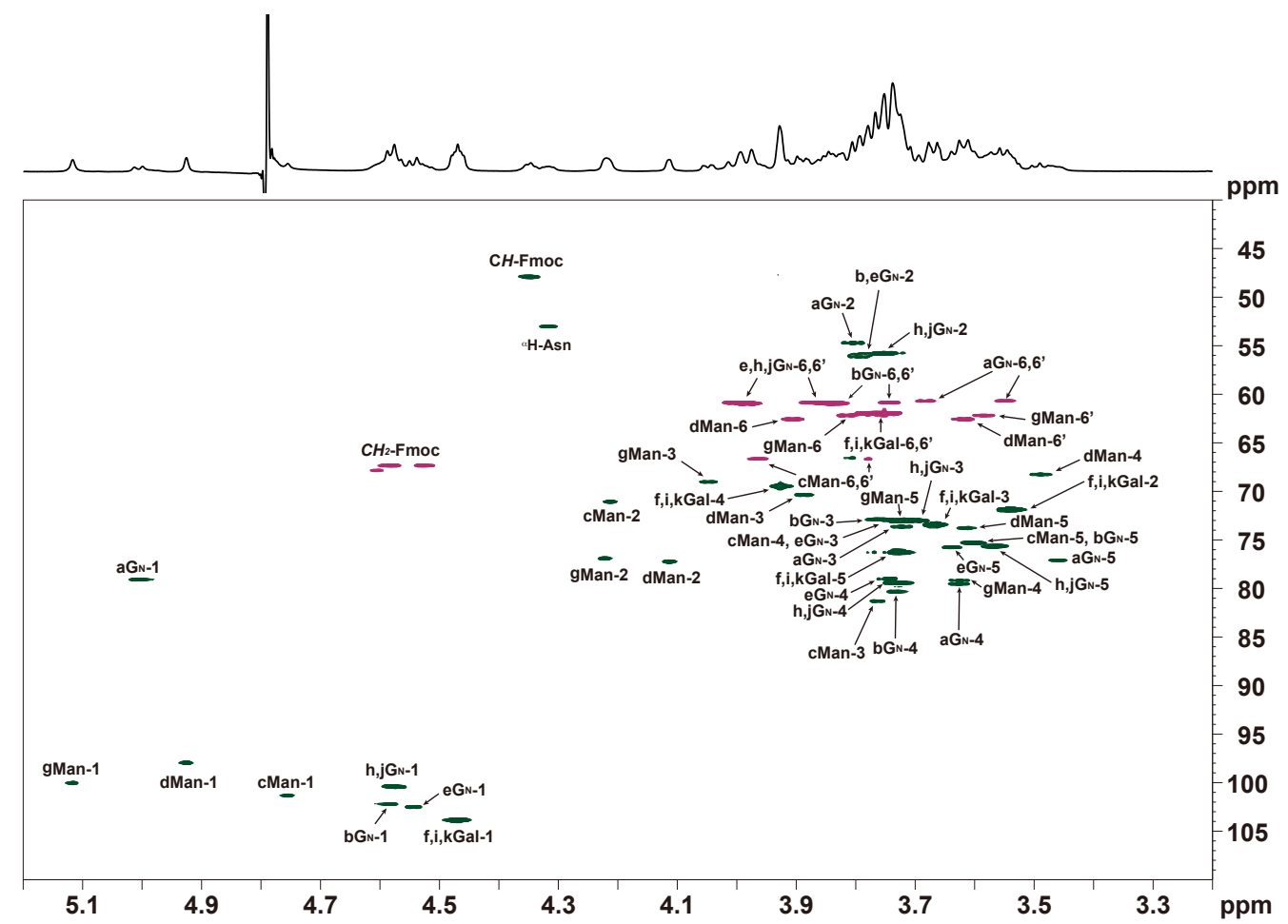

All chemical shifts were assigned based on a previous report ${ }^{6}$. 


\subsection{Synthesis of Benzylidene Monosaccharides}

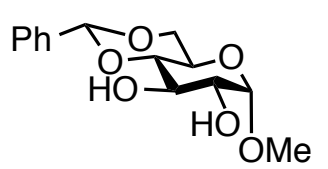

Glc $_{\mathbf{A}}$

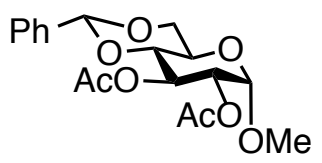

$\mathbf{G l c}_{\mathbf{B}}$

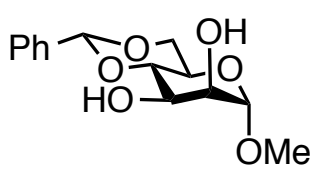

$\operatorname{Man}_{\mathrm{A}}$

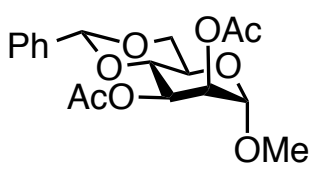

$\operatorname{Man}_{B}$

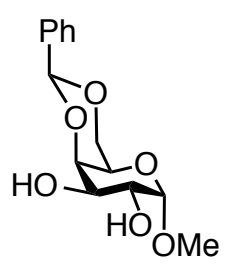

$\mathbf{G a l}_{\mathbf{A}}$

All above methyl 4,6-O-benzylidene- $\alpha$-D-glycosides $\left(\mathrm{Glc}_{\mathrm{A}}\right.$, $\mathrm{Man}_{\mathrm{A}}$, and $\left.\mathrm{Gal}_{\mathrm{A}}\right)$ and their acetate derivatives $\left(\mathrm{Glc}_{\mathrm{B}}\right.$, and $\left.\mathrm{Man}_{\mathrm{B}}\right)$ used for the experiment were prepared as previously reported. ${ }^{7-9}$ Methyl 2,3-di- $O$-acetyl-4,6- $O$-benzylidene- $\alpha$-D-galactoside was synthesized as follow.

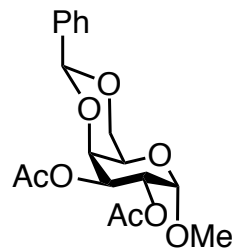

\section{Methyl 2,3-di- $O$-acetyl-4,6- $O$-benzylidene- $\alpha$-D-galactoside}

Methyl 4,6- $O$-benzylidene- $\alpha$-D-galactoside (495 mg, $1.8 \mathrm{mmol}$ ) was dissolved in $17.6 \mathrm{~mL}$ of $\mathrm{Ac}_{2} \mathrm{O}$ /pyridine (1:1), and the resulting mixture was stirred at rt. After 6 hours, $\mathrm{MeOH}(10 \mathrm{~mL})$ was added to the mixture on an ice bath. After elevation of the temperature to $\mathrm{rt}$, the mixture was evaporated and azeotropically dried with toluene. The resulting residue was purified by silica gel column chromatography (hexane $/$ EtOAc $=1: 2, \phi=1.8 \mathrm{~cm}, 1=15 \mathrm{~cm}$ ). The resulting residue was further recrystallized from ethanol and petroleum ether to give $\mathbf{G a l}_{\mathbf{B}}$ (525 $\mathrm{mg}, 1.43$ mmol, $82 \%)$.

$[\mathrm{a}]_{\mathrm{D}}^{25} 189.5\left(c 0.50 \mathrm{CHCl}_{3}\right)$

${ }^{1}$ H NMR (400 MHz, $\left.\mathbf{C D C l}_{3}\right)$ : 7.57-7.47 (m, 2H), 7.42-7.32 (m, 3H), 5.52 (s, 1H), 5.37 (dd, 1H, $J=10.88,3.1 \mathrm{~Hz}), 5.32(\mathrm{dd}, 1 \mathrm{H}, J=10.87,3.11 \mathrm{~Hz}), 5.09(\mathrm{~d}, 1 \mathrm{H}, J=3.02 \mathrm{~Hz}), 4.47(\mathrm{dd}, 1 \mathrm{H}, J$ $=3.24,1.11 \mathrm{~Hz}), 4.28(\mathrm{dd}, 1 \mathrm{H}, J=12.51,1.56 \mathrm{~Hz}), 4.07(\mathrm{dd}, 1 \mathrm{H}, J=12.56,1.68 \mathrm{~Hz}), 3.75$ (brddd, 1H), 3.43 (s, 3H), 2.09 (s, 3H), 2.08 (s, 3H).

${ }^{13}$ C NMR (100 MHz, $\mathbf{C D C l}_{3}$ ): 170.77, 170.33, 137.68, 129.15, 128.31, 126.36, 100.99, 97.89, $74.05,69.22,68.68,68.22,62.16,55.67,21.08,20.98$.

HRMS (ESI): calcd for $\mathrm{C}_{18} \mathrm{H}_{22} \mathrm{O}_{8}[\mathrm{M}+\mathrm{Na}]^{+} 389.1207$, found for $\mathrm{m} / z 389.1206[\mathrm{M}+\mathrm{Na}]^{+}$. 


\subsection{Investigation of Acidic Hydrolysis of Benzylidene Groups}

Six substrates prepared above $(10 \mu \mathrm{mol})$ were dissolved in $1.0 \mathrm{~mL}$ of $60 \% \mathrm{~d}_{3}$-AcOD in $\mathrm{D}_{2} \mathrm{O}$, respectively. The resulting mixtures were kept at $\mathrm{rt} .{ }^{1} \mathrm{H}$ NMR spectra were recorded time-dependently (number of scan: 16, dummy scan: 4). Hydrolysis yield (percentage) was determined as "the integration of ${ }^{1} \mathrm{H}$ signal of a product / (the integration of ${ }^{1} \mathrm{H}$ signal of a product + the integration of ${ }^{1} \mathrm{H}$ signal of a starting material)" (Figure $4 \mathrm{a}, \mathrm{b}$ ). An anomeric proton region was used for the estimation of benzylidene derivatives without acetyl groups, and a methyl proton region of methyl glycoside was used for the estimation of its acetate derivatives.

Competitive hydrolysis experiments were performed using a mixture of glucoside, mannoside, and galactoside $\left(10 \mu \mathrm{mol}\right.$, respectively) in $1.0 \mathrm{~mL}$ of $60 \% \mathrm{~d}_{3}$-AcOD in $\mathrm{D}_{2} \mathrm{O}$, and the reaction was monitored as above (Figure 4c, d). Because of the signal overlap in ${ }^{1} \mathrm{H}$ NMR, we did not estimate the hydrolysis yield in these experiments. 


\section{Figures}

3.1

(a)

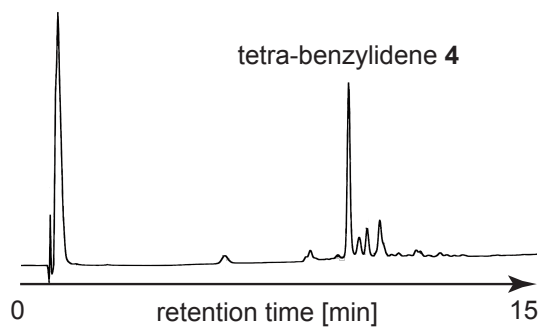

(b)

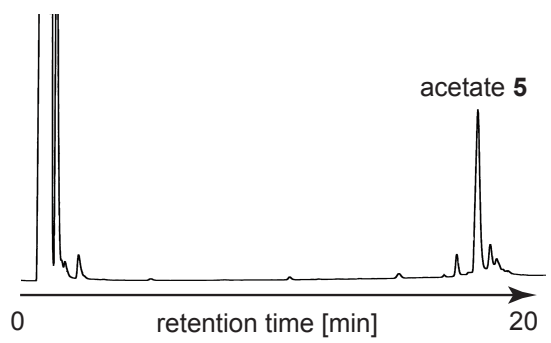

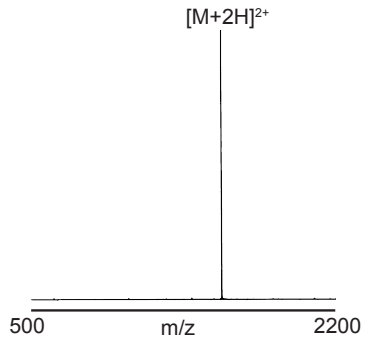

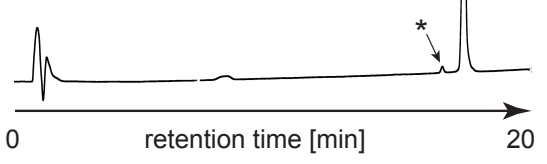

Figure S1. (a) RP-HPLC profile of crude tetra-benzylidene derivative 4 after completion of the benzylidene acetalation. (b) RP-HPLC profile of crude acetate 5 after acetylation of 4 . (c) RP-HPLC profile and ESI-MS spectrum of purified acetate 5. *Aspartimide byproduct was observed as $1.6 \%$ area on HPLC analysis. This aspartimide formation occurred either right after HPLC purification or during HPLC analysis. 


\section{2}

(a)

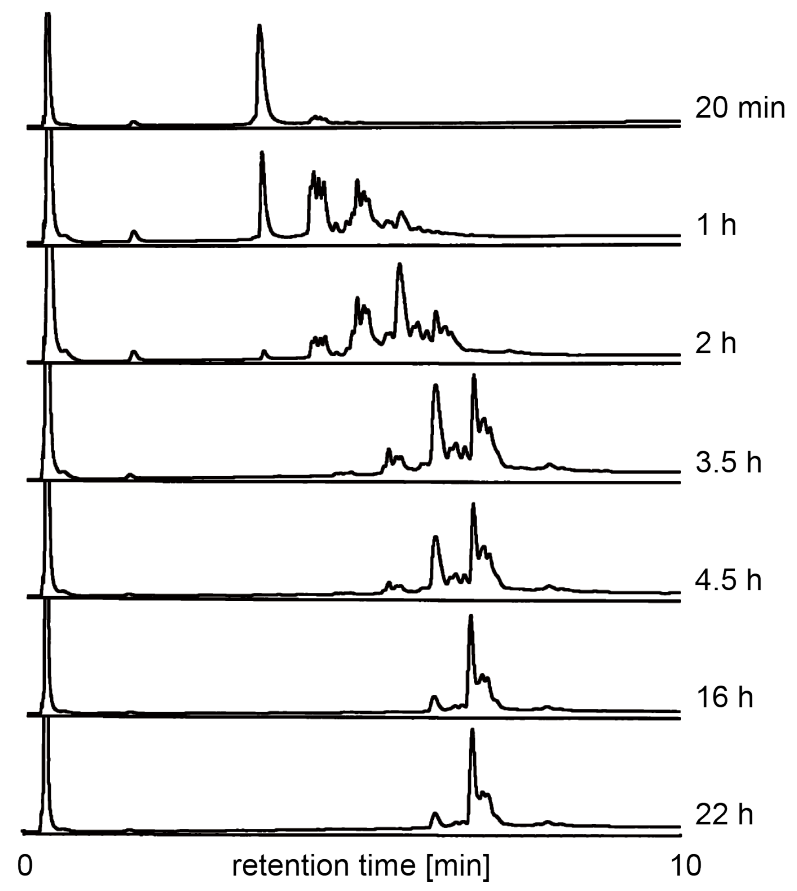

(b)

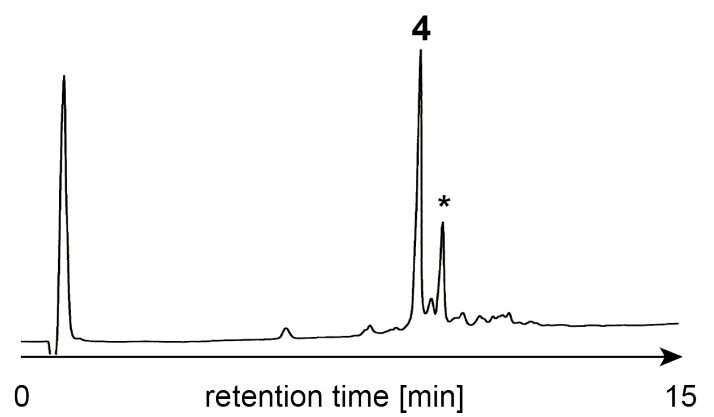

Figure S2. Kinetic benzylidenation of oligosaccharide 5.

(a) Time-dependent RP-UltraFast-HPLC chromatograms (low resolution) monitoring the benzylidenation reaction. This reaction was conducted with camphor-10-sulfonic acid (5 equiv) and benzaldehyde dimethyl acetal (20 equiv) at $37{ }^{\circ} \mathrm{C}$. (b) A typical HPLC profile of benzylidenation reaction with high resolution. The conversion yield of desired tetra-benzylidene derivative 4 is $50-70 \%$ based on the peak areas of the HPLC chromatogram. *MS analysis suggested that this side-product might be a regio- or stereo-isomer of benzylidene derivatives, but the structure was not determined furthermore. 
(a)

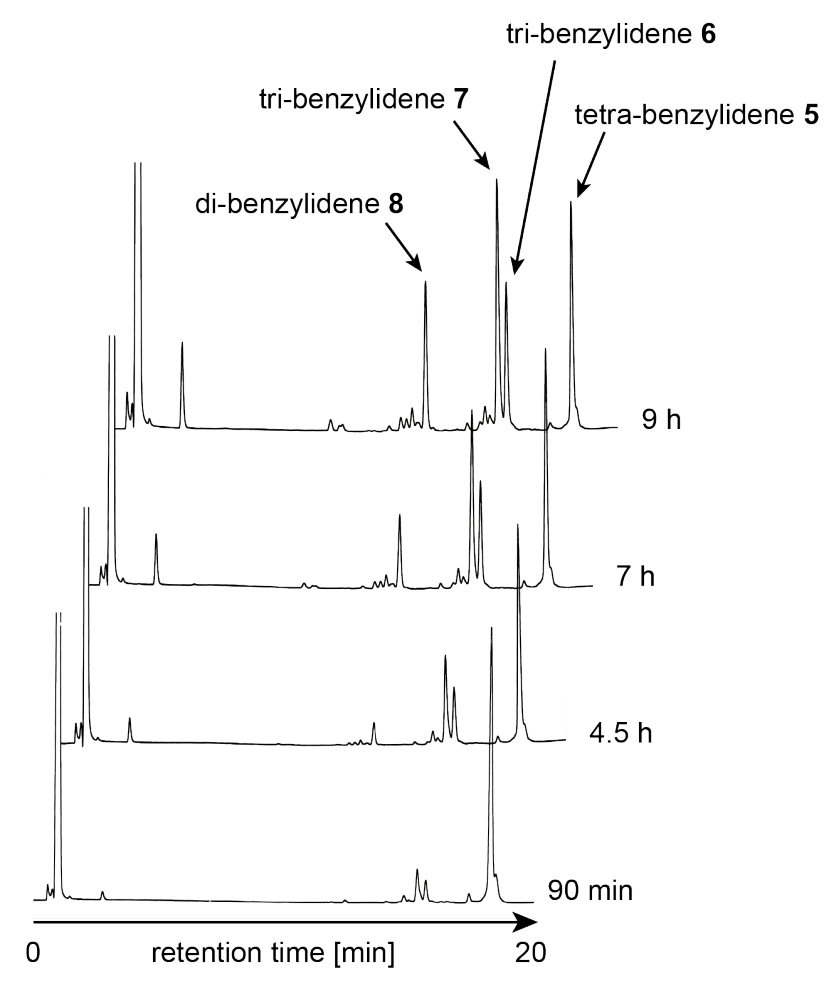

(b)

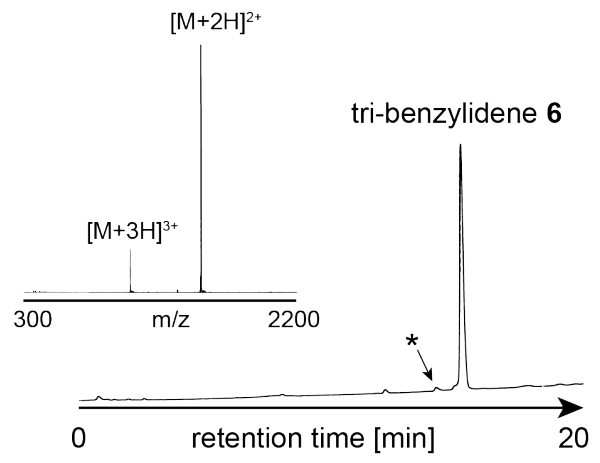

(c)

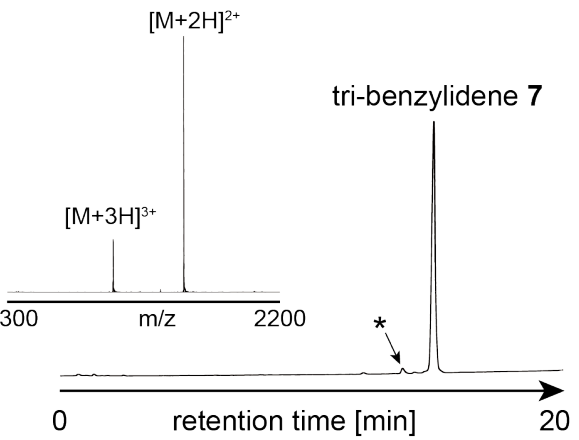

Figure S3. Kinetic and selective removal of benzylidene groups on mannoside residues of 5.

(a) Typical time-dependent RP-HPLC chromatograms monitoring the benzylidene-removal reaction from tetra-benzylidene 5. Based on MS analysis, minor peaks observed were thought to be isomers and unknown products, but we could not determine their structure because of the small quantities. (b) HPLC profile and ESI-MS spectrum of purified tri-benzylidene derivative 6. *Aspartimide byproduct was observed as $1.4 \%$ area on HPLC analysis. This aspartimide was occurred either right after HPLC purification or during HPLC analysis. (c) HPLC profile and ESI-MS spectrum of purified tri-benzylidene derivative 7. *Aspartimide byproduct was observed as $2.0 \%$ area on HPLC analysis. This aspartimide formation occurred either right after HPLC purification or during HPLC analysis. 


\section{4}

(a)
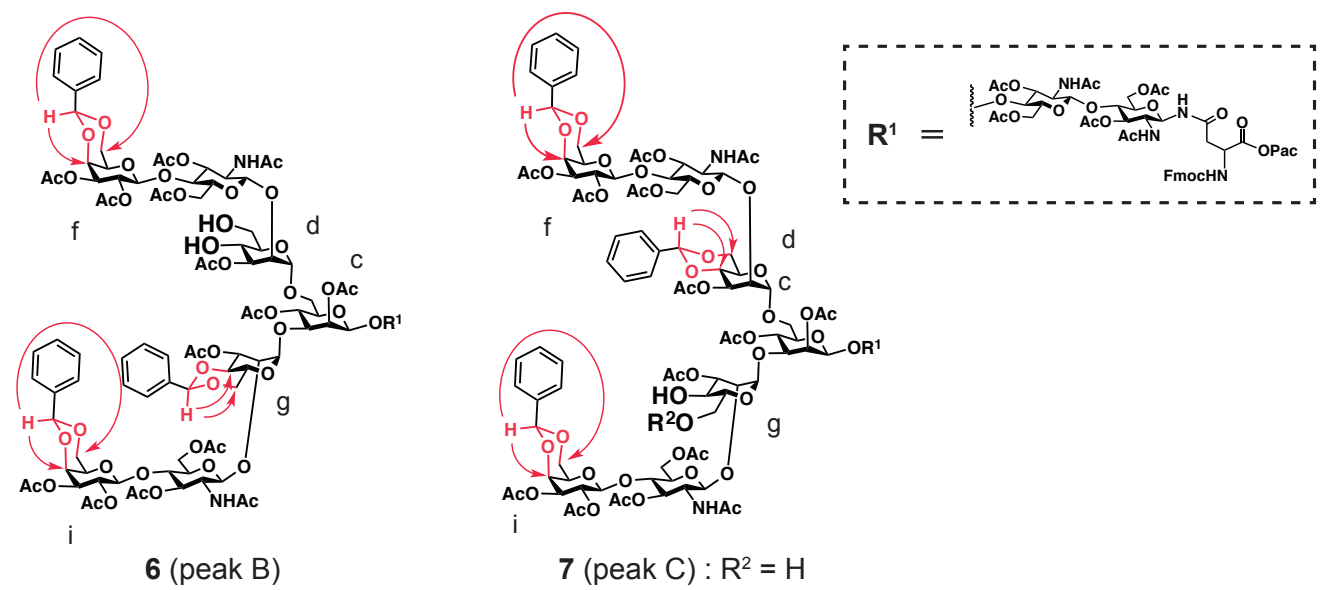

(b)

(c)
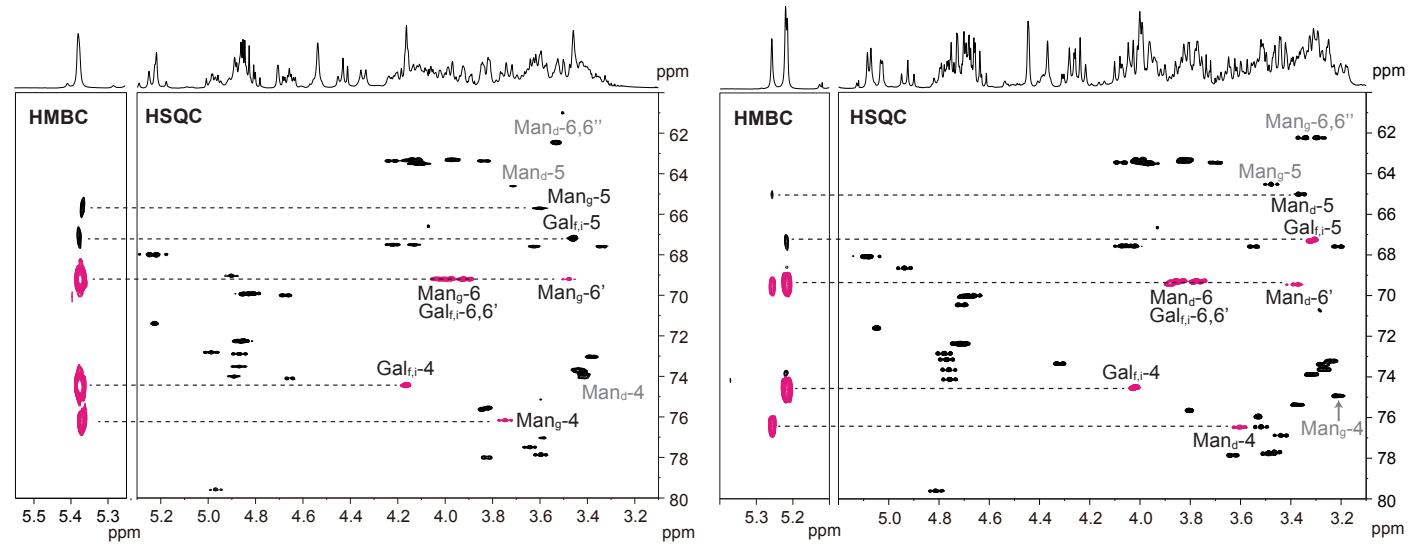

Figure S4. (a) The structures of partially debenzylidenated oligosaccharides 6 and 7. The observed HMBC correlation signals are shown by red arrows. (c) Characterization of glycosyl acceptor 6. The selective regions of HSQC (right square) and HMBC (left square) spectra of 6 are shown. (d) Characterization of diol 7. The selective regions of HSQC (right square) and HMBC (left square) spectra of 7 are shown. 
(a)

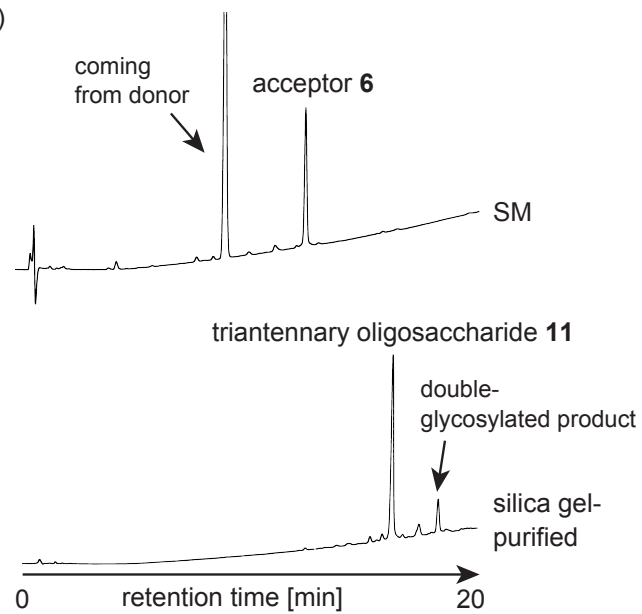

(b)

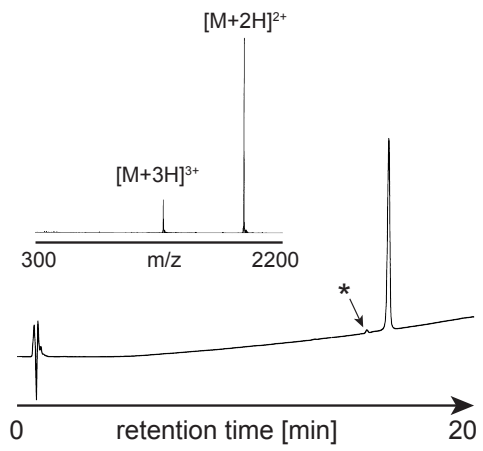

Figure S5. (a) RP-HPLC profiles of the glycosylation reaction, starting materials (upper) and silica gel-purified material (bottom). (b) HPLC profile and ESI-MS spectrum of HPLC-purified triantennary glycan 11. *Aspartimide byproduct was observed as $1.4 \%$ area on HPLC analysis. This aspartimide formation occurred either right after HPLC purification or during HPLC analysis. 
3.6
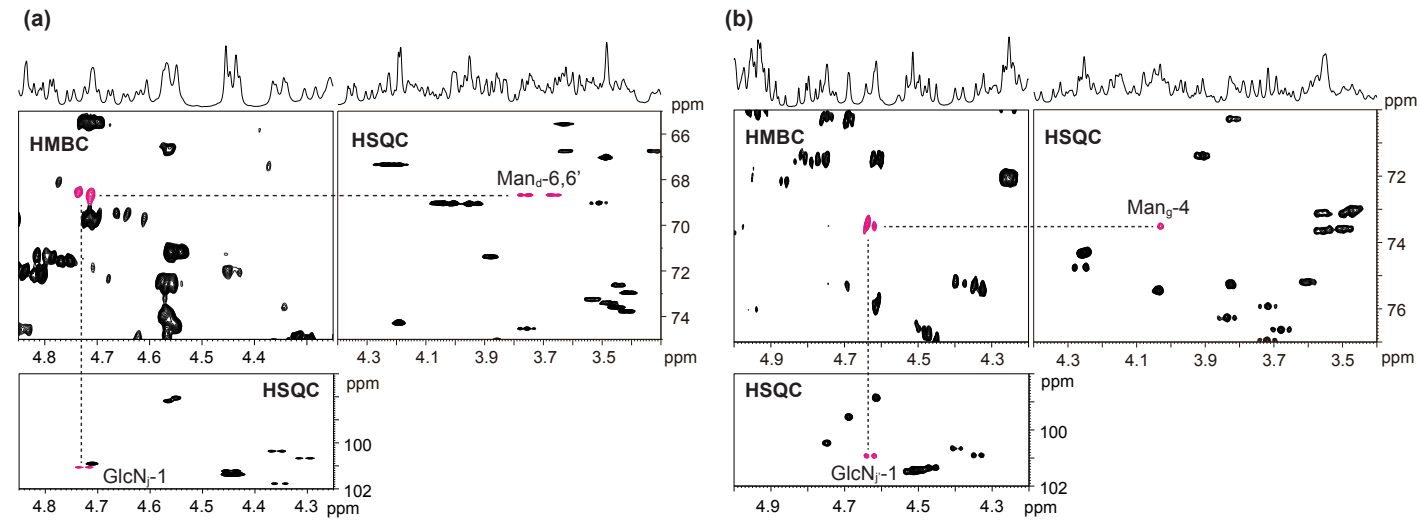

Figure S6. (a) The selective regions of HSQC and HMBC spectra of 11. The HMBC correlation signal between the C-6 of $\mathrm{Man}_{\mathrm{d}}$ and the H-1 of $\mathrm{GlcN}_{\mathrm{j}}$ (dotted line) was observed. The newly synthesized $\beta$-linkage was determined by the coupling constant observed for $\mathrm{H}-1$ and $\mathrm{H}-2$ in $\mathrm{GlcN}_{\mathrm{j}}(8.5 \mathrm{~Hz})$. (b) The selective regions of HSQC and HMBC spectra of 12. The HMBC correlation signal between the C-4 of $\mathrm{Man}_{\mathrm{d}}$ and the $\mathrm{H}-1$ of $\mathrm{GlcN}_{\mathrm{j}}$, (dotted line) was observed. The newly synthesized $\beta$-linkage was determined by the coupling constant observed for $\mathrm{H}-1$ and $\mathrm{H}-2$ in $\mathrm{GlcN}_{\mathrm{j}}$, $(8.3 \mathrm{~Hz})$. 
3.7

(a)

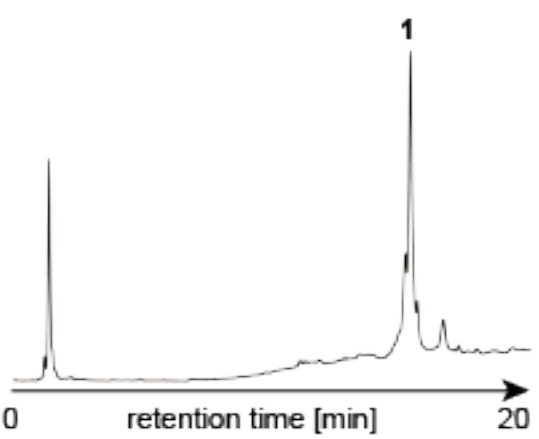

(b)

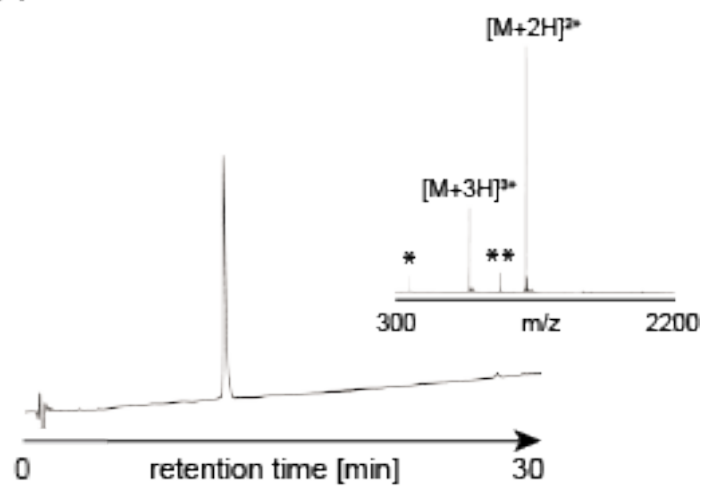

Figure S7. (a) RP-HPLC profile of crude $\beta$-1,6-branched triantennary oligosaccharide 1 after completion of the deprotection reactions. (b) HPLC profile and ESI-MS spectrum of purified Asn-linked $\beta$-1,6-branched triantennary oligosaccharide 1. Asterisk *and ** indicate fragmentation peaks during mass analysis and correspond to LacNAc and biantennary asialo-oligosaccharide, respectively. 
3.8

(a)

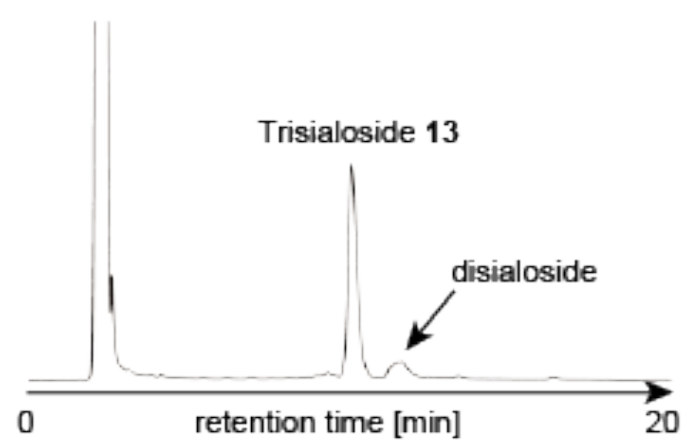

(b)

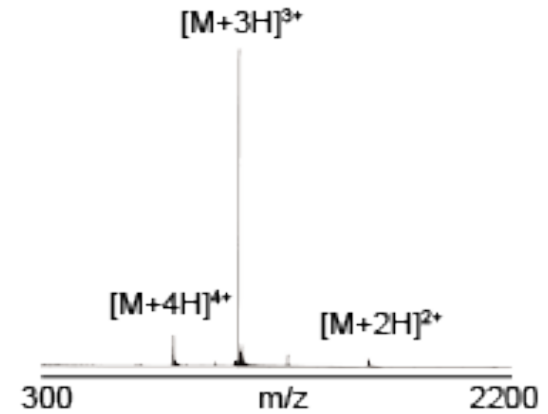

Figure S8. (a) RP-HPLC profile of crude tri-sialyl oligosaccharide $\mathbf{1 3}$ after completion of the sialyltransferase reaction. (b) ESI-MS spectrum of purified triantennary sialyl-tetradecasaccharide $\mathbf{1 3}$. 
3.9

(a)

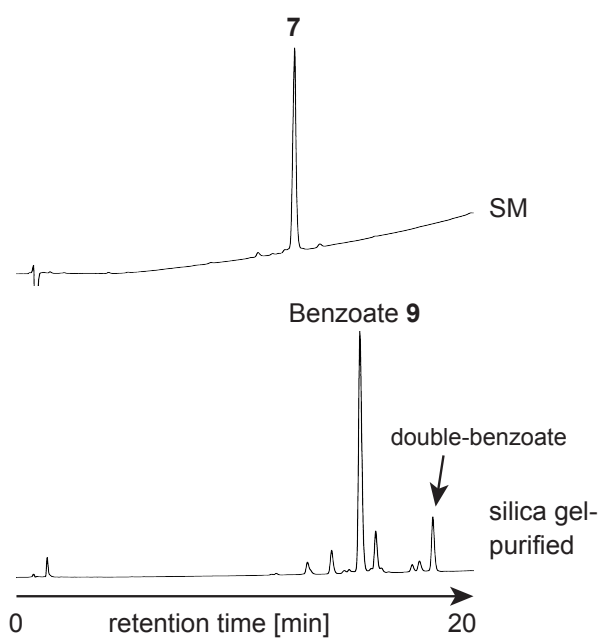

(b)
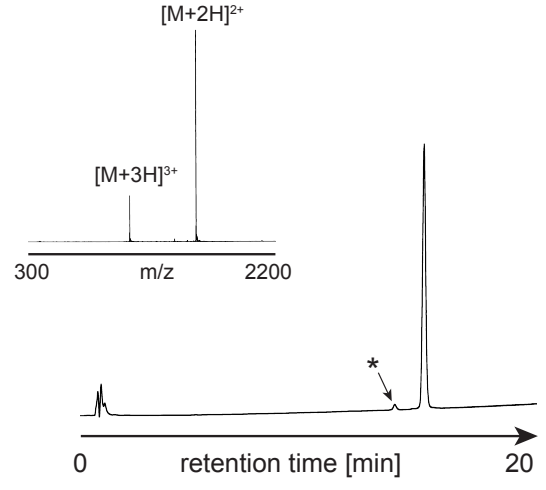

Figure S9. (a) RP-HPLC profiles of the benzoylation reaction of compound 7; starting material (upper) and silica gel-purified material (bottom). (b) HPLC profile and ESI-MS spectrum of HPLC-purified benzoate 9. *Aspartimide byproduct was observed as $1.8 \%$ area on HPLC analysis. This aspartimide formation occurred either right after HPLC purification or during HPLC analysis. 


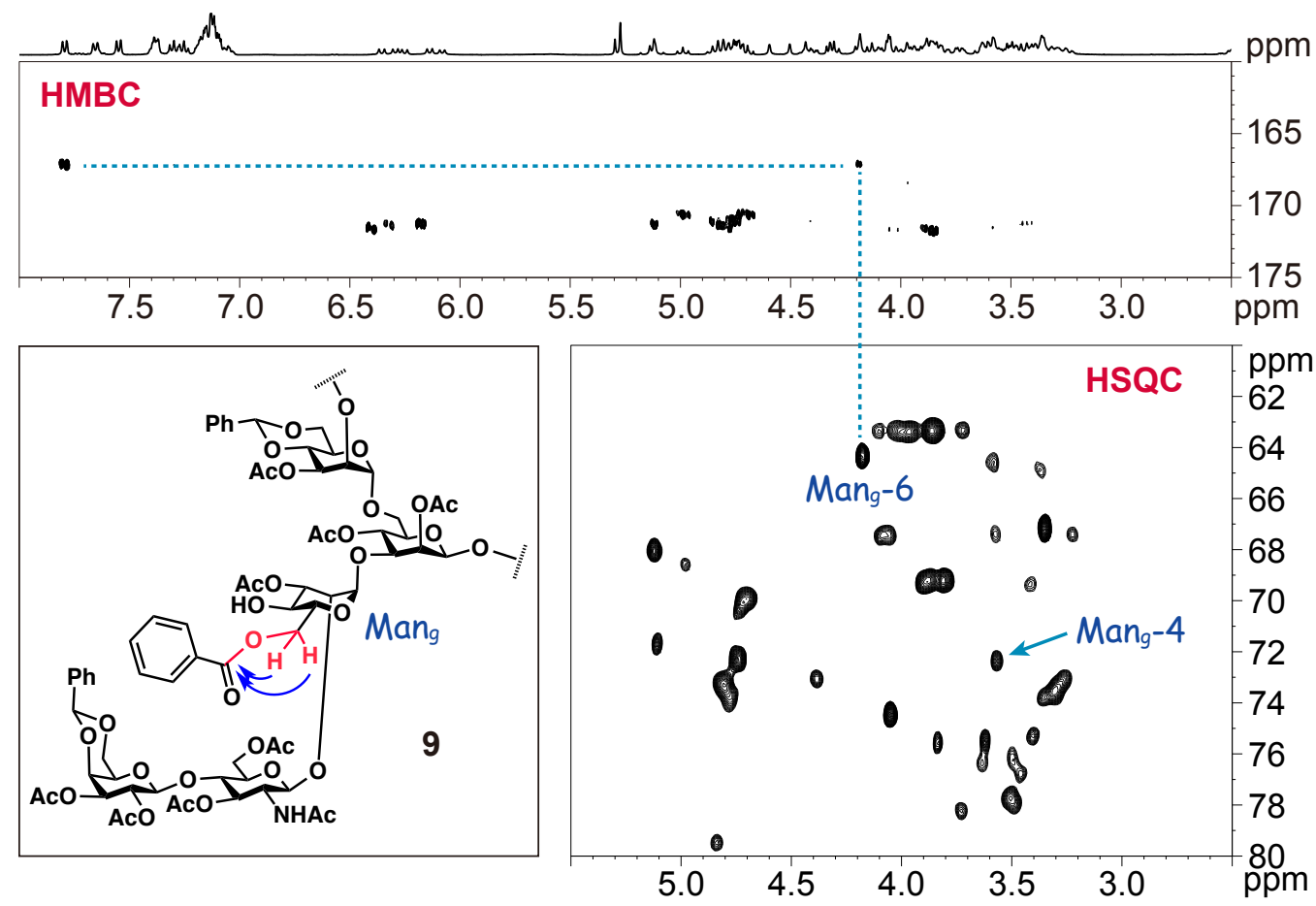

Figure S10. (a) Selected regions of HMBC and HSQC spectra. Chemical shift of carbonyl carbon of benzoyl group was assigned at $167 \mathrm{ppm}$ because this carbon shows correlations with aromatic $o$-protons. HMBC correlation from carbonyl carbon of benzoyl group to H-6 of $\mathrm{Man}_{\mathrm{g}}$ was observed. HSQC signal of $\mathrm{Man}_{\mathrm{g}}-6$ shows down field shift in terms of ${ }^{1} \mathrm{H}$ and ${ }^{13} \mathrm{C}$ after benzoylation reaction. 


\subsection{1}

(a)

(b)
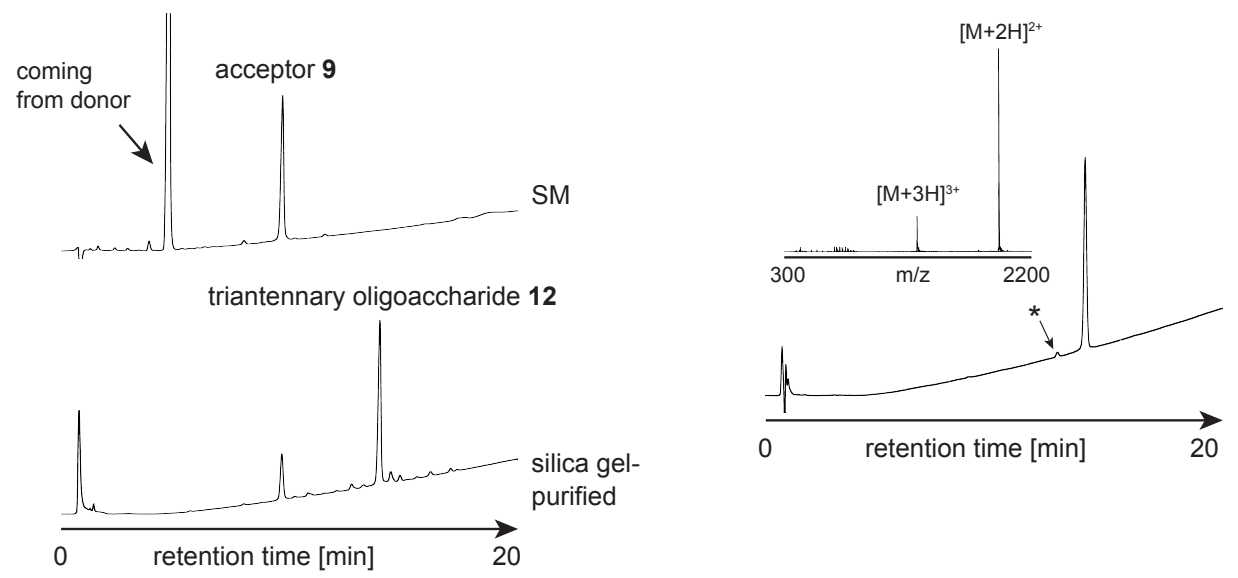

Figure S11. (a) RP-HPLC profiles of the glycosylation reaction; starting materials (upper) and silica gel-purified materials (bottom). (b) HPLC profile and ESI-MS spectrum of HPLC-purified triantennary oligosaccharide 12. *Aspartimide byproduct was observed as $2.3 \%$ area on HPLC analysis. This aspartimide formation occurred either right after HPLC purification or during HPLC analysis. 
3.12

(a)

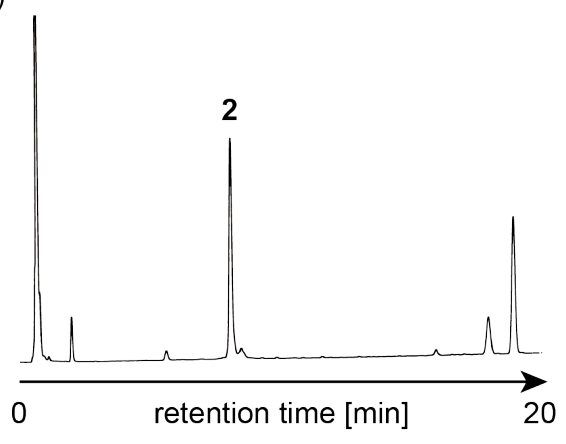

(b)

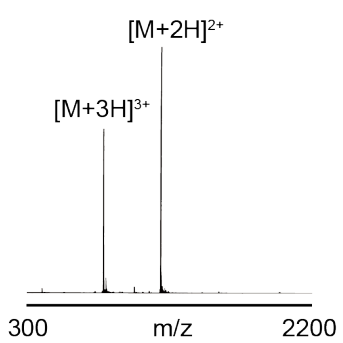

retention time $[\mathrm{min}] \quad 30$

Figure S12. (a) RP-HPLC profile of crude $\beta$-1,4-branched triantennary oligosaccharide 2 after the deprotection reactions. (b) HPLC profile and ESI-MS spectrum of purified Asn-linked $\beta$-1,4-branched triantennary complex-type oligosaccharide $\mathbf{2}$. 


\subsection{3}

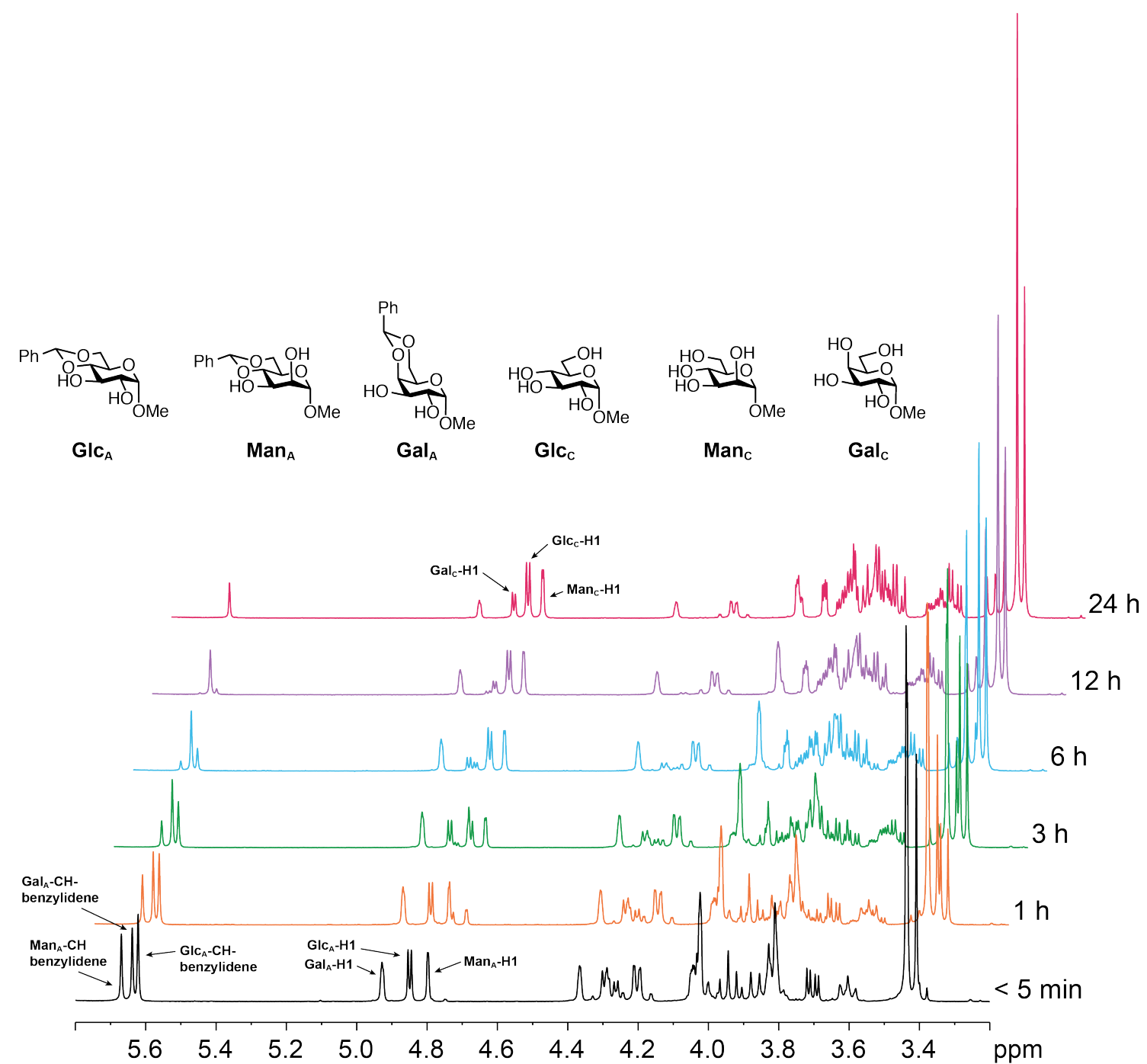

Figure S13. The monitoring of the competitive hydrolysis reaction of methyl 4,6-O-benzylidene- $\alpha$-D-glycosides by ${ }^{1} \mathrm{H}$ NMR. Gal $-\mathrm{H} 1, \mathrm{Glc}_{\mathrm{A}}-\mathrm{H} 1$, and $\mathrm{Man}_{\mathrm{A}}-\mathrm{H} 1$ are the anomeric signals of substrates, and $\mathrm{Gal}_{\mathrm{C}}-\mathrm{H} 1, \mathrm{Glc}_{\mathrm{C}}-\mathrm{H} 1$, and $\mathrm{Man}_{\mathrm{C}}-\mathrm{H} 1$ are the anomeric signals of products. $\mathrm{Gal}_{\mathrm{A}}-\mathrm{CH}$-benzylidene, $\mathrm{Glc}_{\mathrm{A}}-\mathrm{CH}$-benzylidene and $\mathrm{Man}_{\mathrm{A}}-\mathrm{CH}$-benzylidene indicate the signal of benzylidene acetal protons of substrates. After $24 \mathrm{~h}$, the benzylidene group of galactoside was found to remain. The expanded spectrum is shown in Figure 4c. 


\subsection{4}

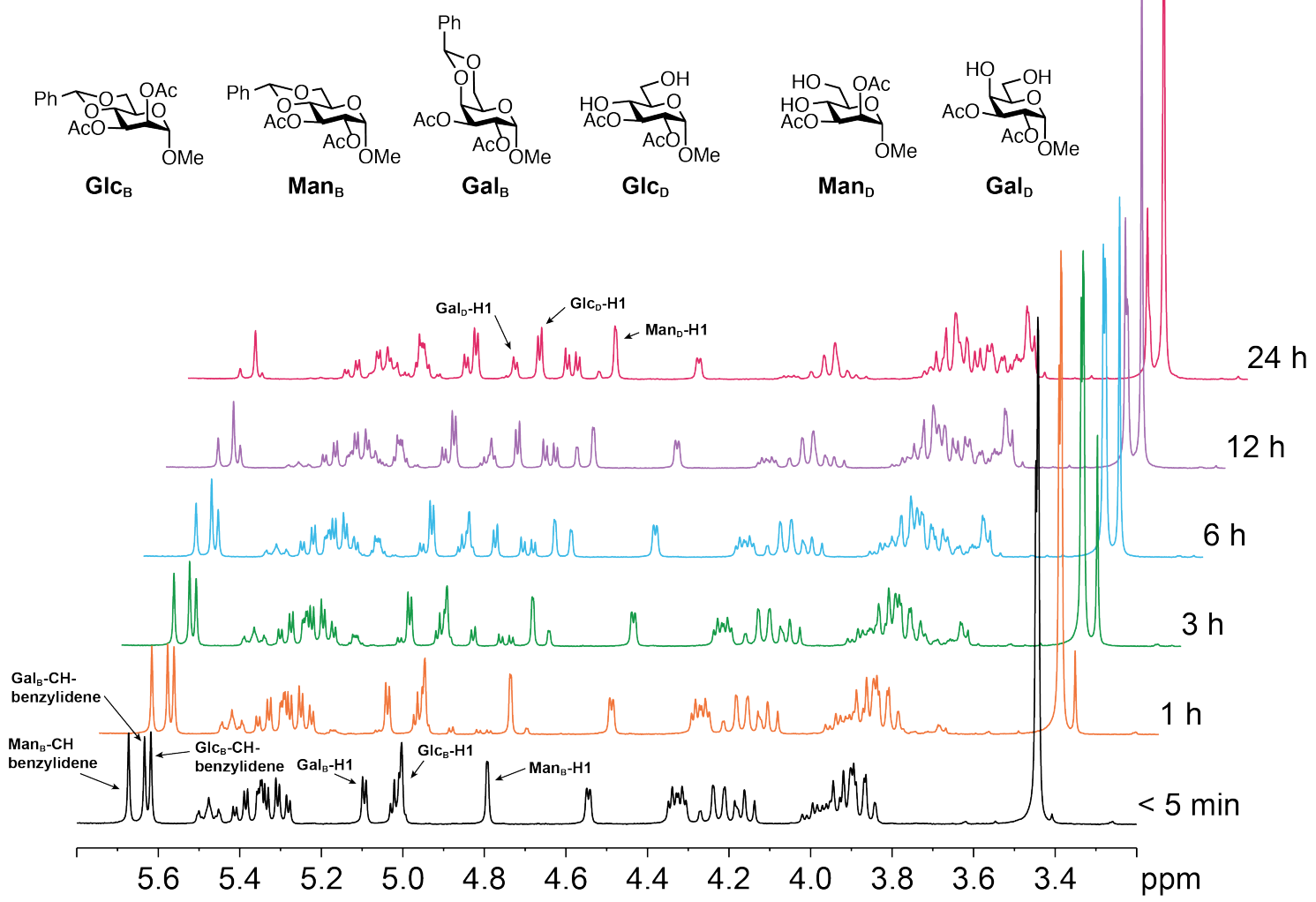

Figure S14. The monitoring of the competitive hydrolysis reaction of methyl 2,3-di- $O$-acetyl-4,6- $O$-benzylidene- $\alpha$-D-glycosides by ${ }^{1} \mathrm{H}$ NMR. Gal $-\mathrm{H} 1, \mathrm{Glc}_{\mathrm{B}}-\mathrm{H} 1$, and $\mathrm{Man}_{\mathrm{B}}-\mathrm{H} 1$ are the anomeric signals of acetylated substrates and $\mathrm{Gal}_{\mathrm{D}}-\mathrm{H} 1, \mathrm{Glc}_{\mathrm{D}}-\mathrm{H} 1$ and $\mathrm{Man}_{\mathrm{D}}-\mathrm{H} 1$ are anomeric signals of products. $\mathrm{Gal}_{\mathrm{B}}-\mathrm{CH}$-benzylidene, $\mathrm{Glc}_{\mathrm{B}}-\mathrm{CH}$-benzylidene, and $\mathrm{Man}_{\mathrm{B}}-\mathrm{CH}$-benzylidene indicate the signal of benzylidene acetal protons of substrates. After $24 \mathrm{~h}$, the benzylidene group of galactoside was found to clearly remain. The expanded spectrum is shown in Figure 4d. 


\section{Examples of Other Investigation of the Reactions}

\subsection{Silylation reaction}

To a solution of Asn-linked biantennary asialo-nonasaccharide $3(9.8 \mathrm{mg}, 5 \mu \mathrm{mol})$ in DMF (1.0 $\mathrm{mL})$ were added imidazole $(20 \mu \mathrm{mol})$ and TBDPSCl $(25 \mathrm{mmol})$. The reaction mixture was stirred at rt. However, reaction did not proceed. After 12 hours, TBDPSCl $(25 \mu \mathrm{mol})$ was added again to the mixture, followed by further addition of TBDPSCl $(193 \mu \mathrm{mol})$. This reaction found to give multiple products as shown in Figure S15-1.

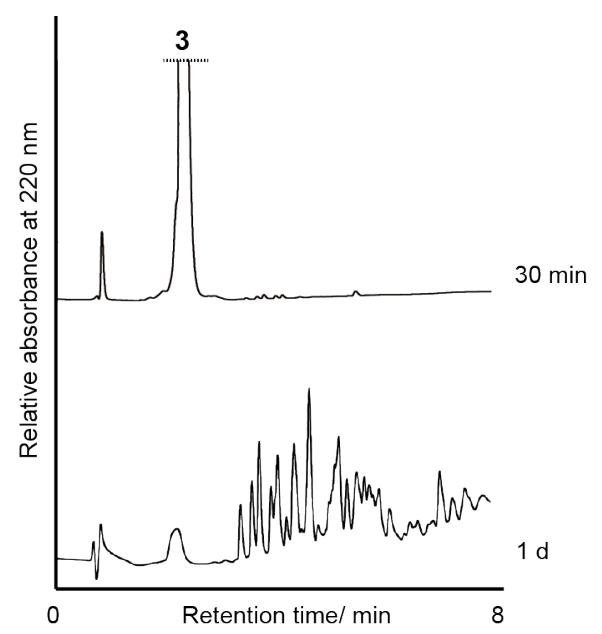

Figure S15-1. HPLC monitoring of silylation. 


\subsection{Tritylation reaction}

To a solution of Asn-linked biantennary asialo-nonasaccharide 3 (10 mg, $5 \mu \mathrm{mol})$ in DMF (630 $\mu \mathrm{L})$ were added ${ }^{i} \operatorname{Pr}_{2} \mathrm{NEt}(25 \mu \mathrm{mol})$ and $\operatorname{TrCl}(25 \mu \mathrm{mol})$. The reaction mixture was stirred at $\mathrm{rt}$. This reaction yielded over 5 derivatives as shown S15-2, and therefore we did not use this protection for the synthesis of triantennary oligosaccharides.

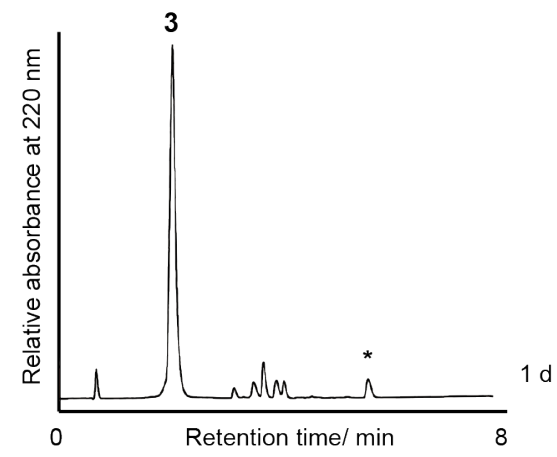

Figure S15-2. Monitoring of tritylation reaction.

* This peak is not an oligosaccharide derivative. 


\subsection{Glycosylation of oligosaccharyl acceptor 6 with disaccharide 10}

A mixture of acceptor $\mathbf{6}(1.7 \mathrm{mg}, 0.6 \mu \mathrm{mol})$ and donor $\mathbf{1 0}(1.0 \mathrm{mg}, 1.2 \mu \mathrm{mol}, 2$ equiv) was azeotropically dried with toluene and kept in vacuo for 2 hours, after which the mixture was dissolved in $\mathrm{CH}_{2} \mathrm{Cl}_{2}(100 \mu \mathrm{L})$ under an atmosphere of Ar, followed by addition of freshly activated $4 \mathrm{~A}$ molecular sieves $\left(13 \mathrm{mg}\right.$ ). The reaction mixture was cooled to $-20{ }^{\circ} \mathrm{C}$, and NIS (4.4 $\mu \mathrm{mol})$ was added. Then, TfOH $\left(0.44 \mu \mathrm{mol}\right.$, with dilution by $\left.\mathrm{CH}_{2} \mathrm{Cl}_{2}\right)$ was added to the mixture, which was stirred at the same temperature for $3.5 \mathrm{~h}$. This reaction afforded undesired by product, aspartimide rather than desired triantennary oligosaccharide 11. Because donor was not enough, the reaction did not finish within $2 \mathrm{~h}$ and the condition of longer reaction time yielded undesired aspartimide derivative.

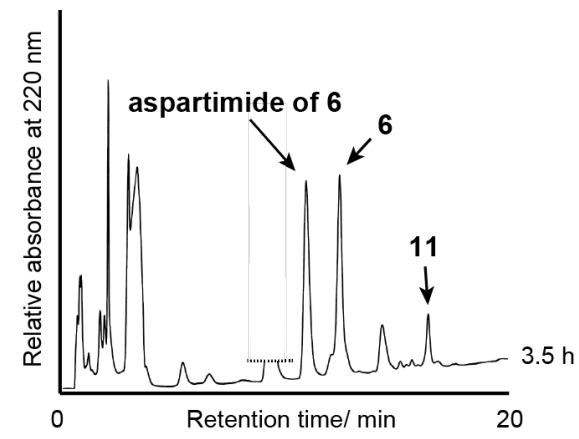

Figure S15-3. Monitoring a glycosylation reaction of 6 under another reaction condition. 


\section{NMR Data}

${ }^{1}$ H NMR of $\mathbf{S 1}$

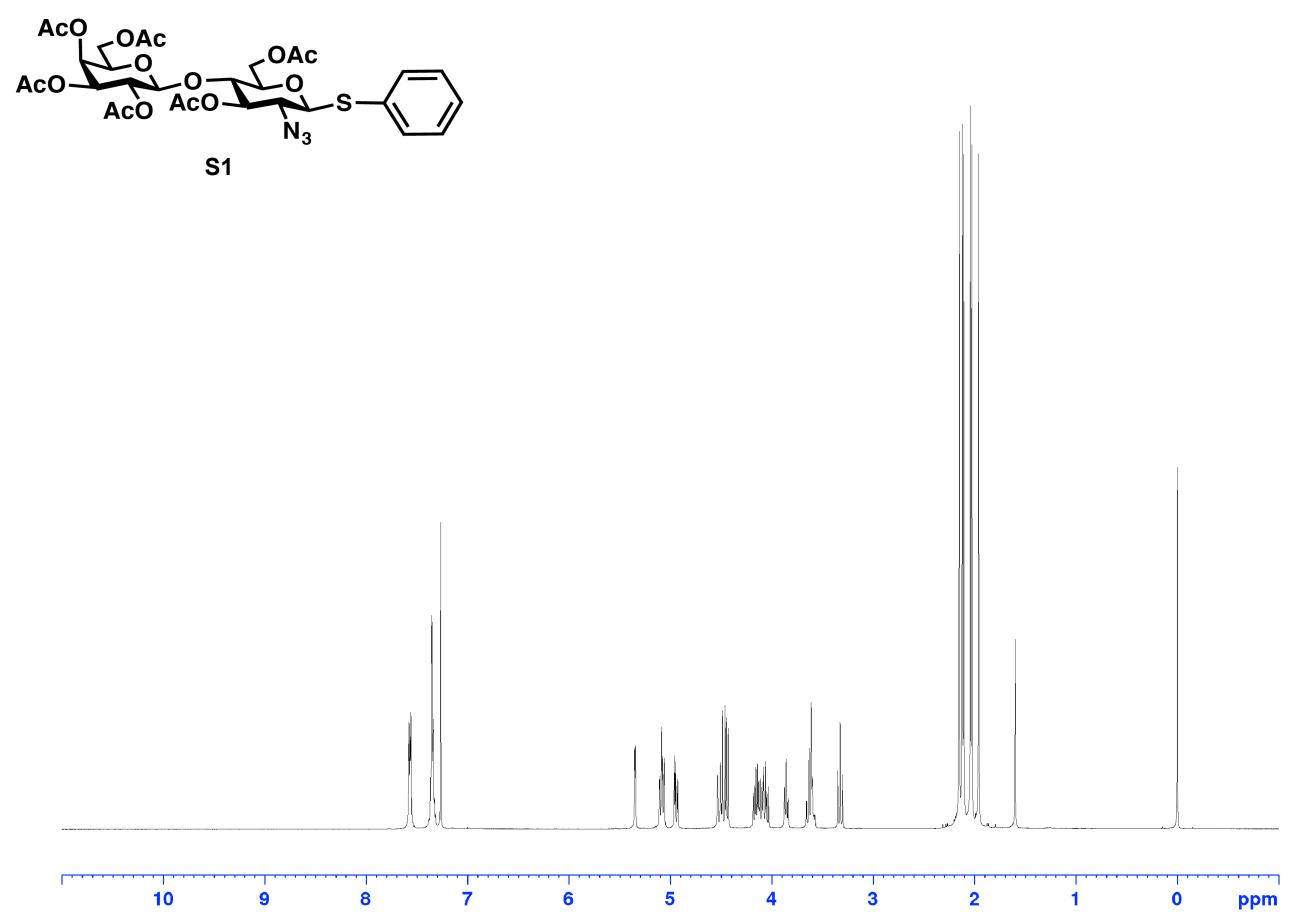

${ }^{13} \mathrm{C}$ NMR of $\mathbf{S 1}$

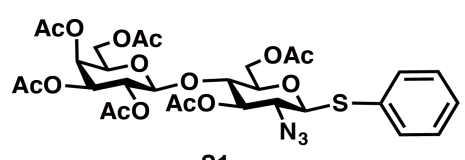

s1

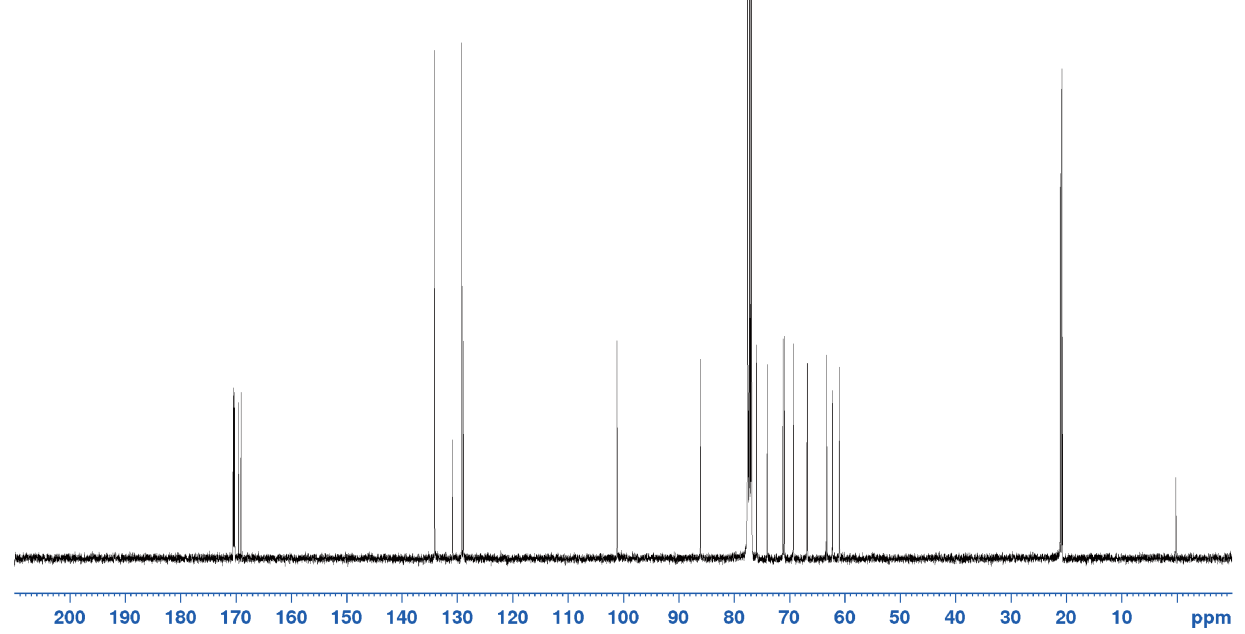


${ }^{1} \mathrm{H}$ NMR of $\mathbf{1 0}$
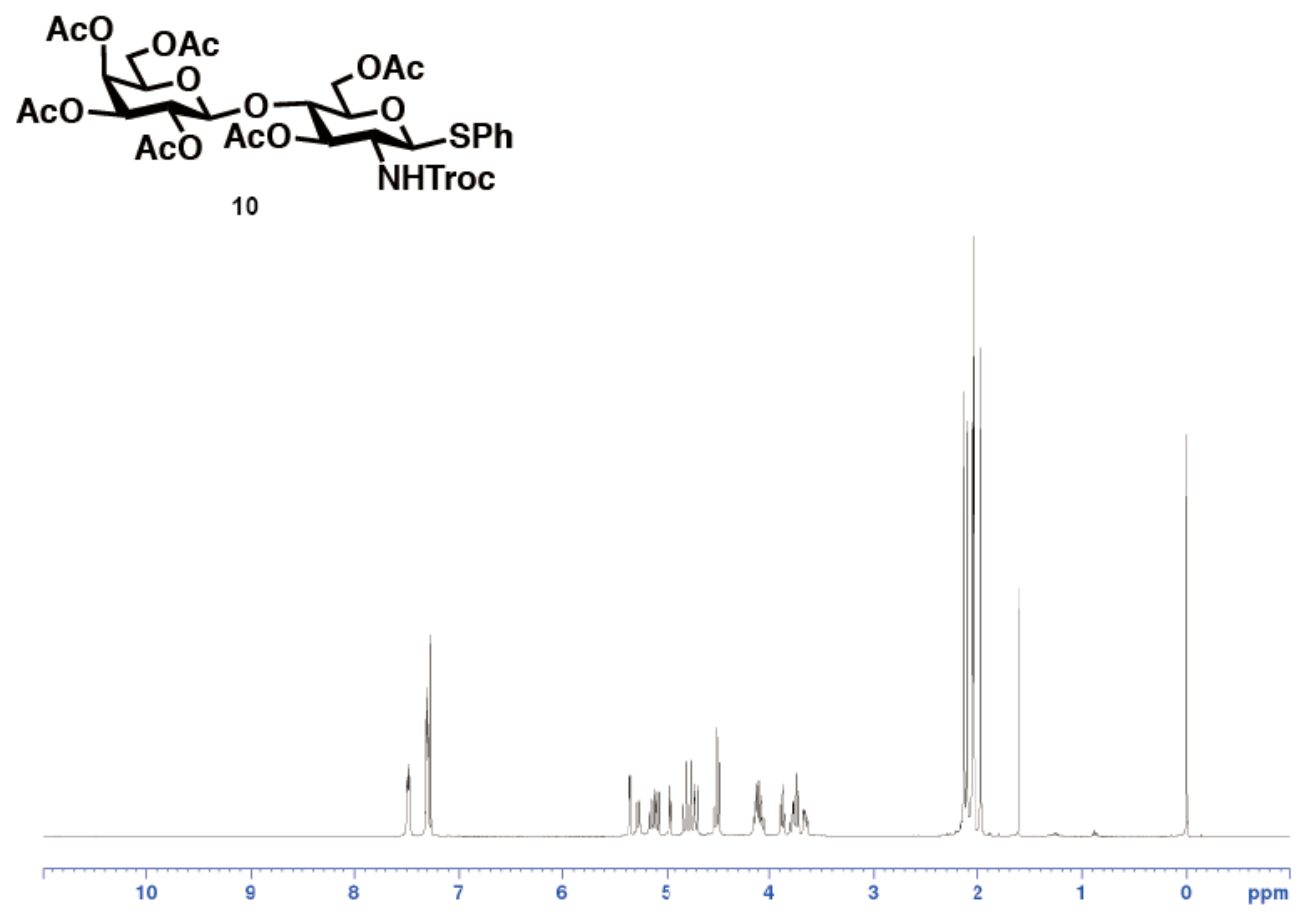

${ }^{13} \mathrm{C}$ NMR of $\mathbf{1 0}$

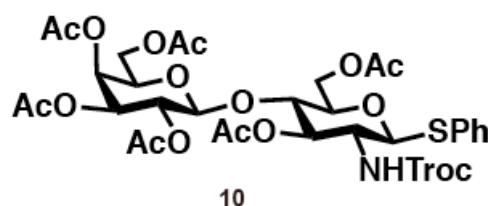

10

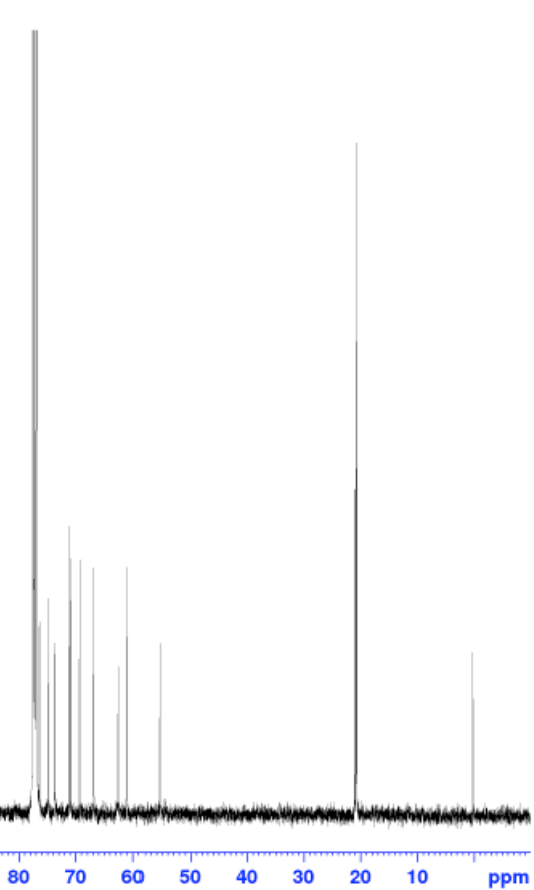


${ }^{1} \mathrm{H}$ NMR of $\mathbf{5}$
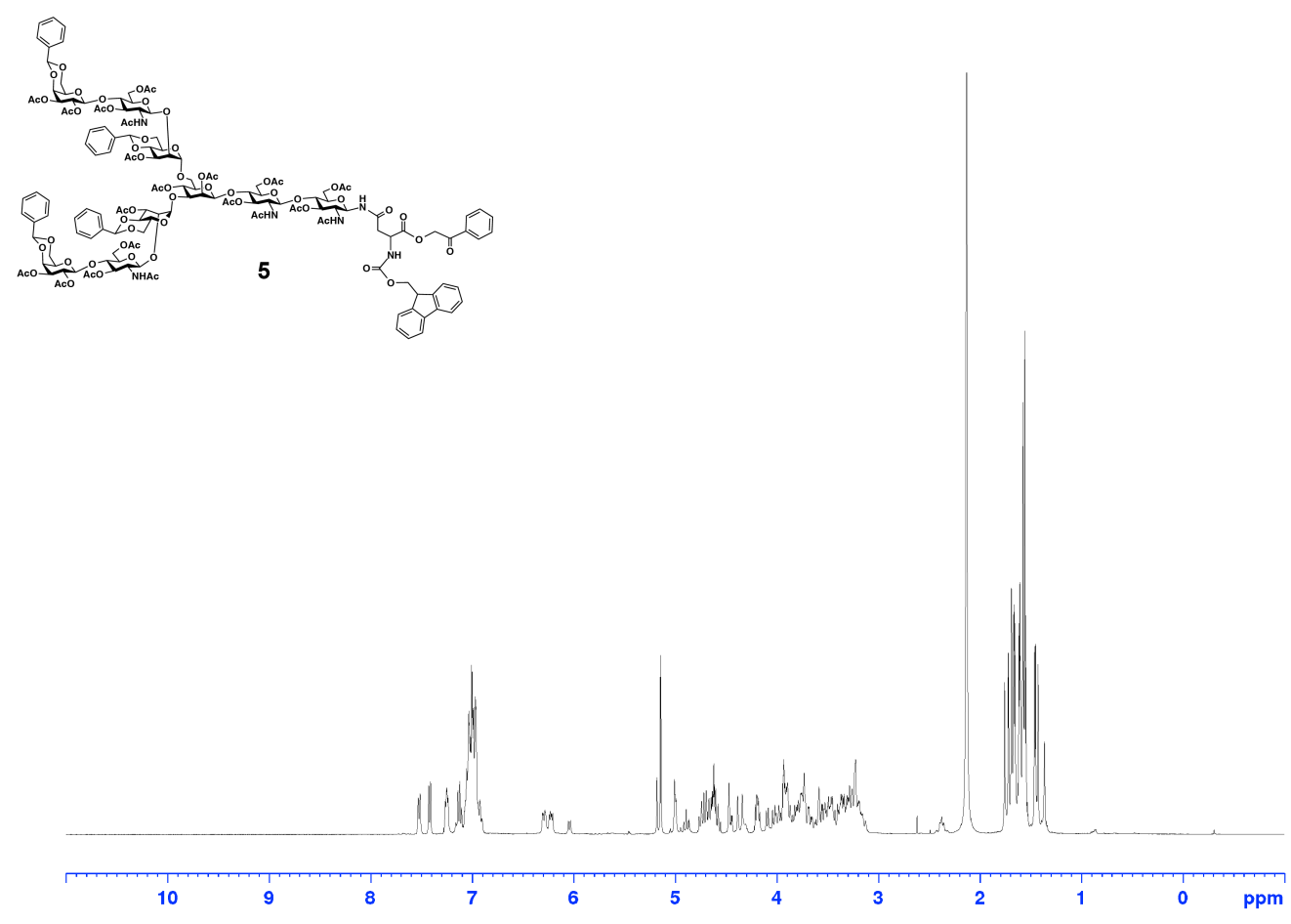

${ }^{13} \mathrm{C}$ NMR of 5

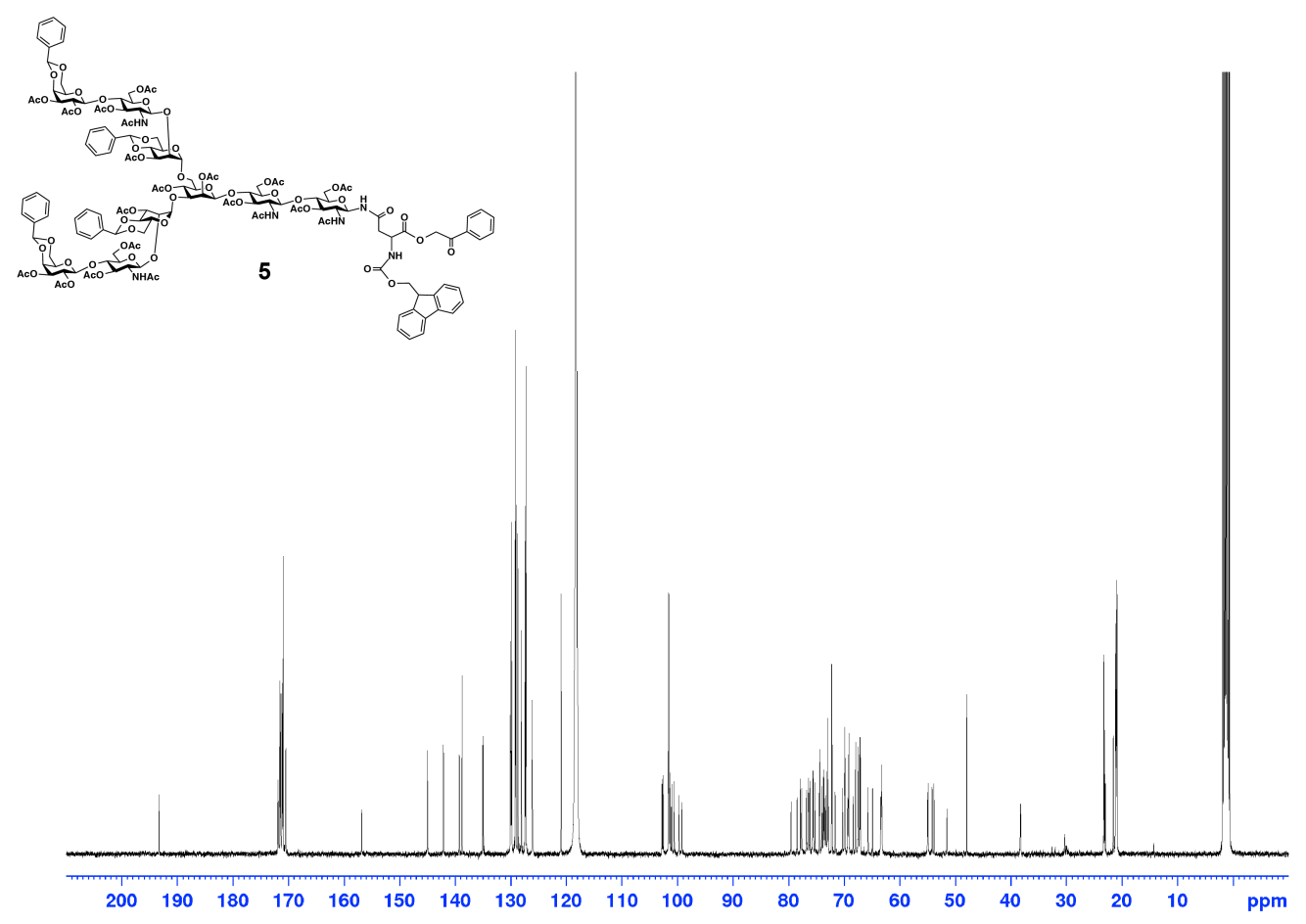

S50 
HSQC of 5

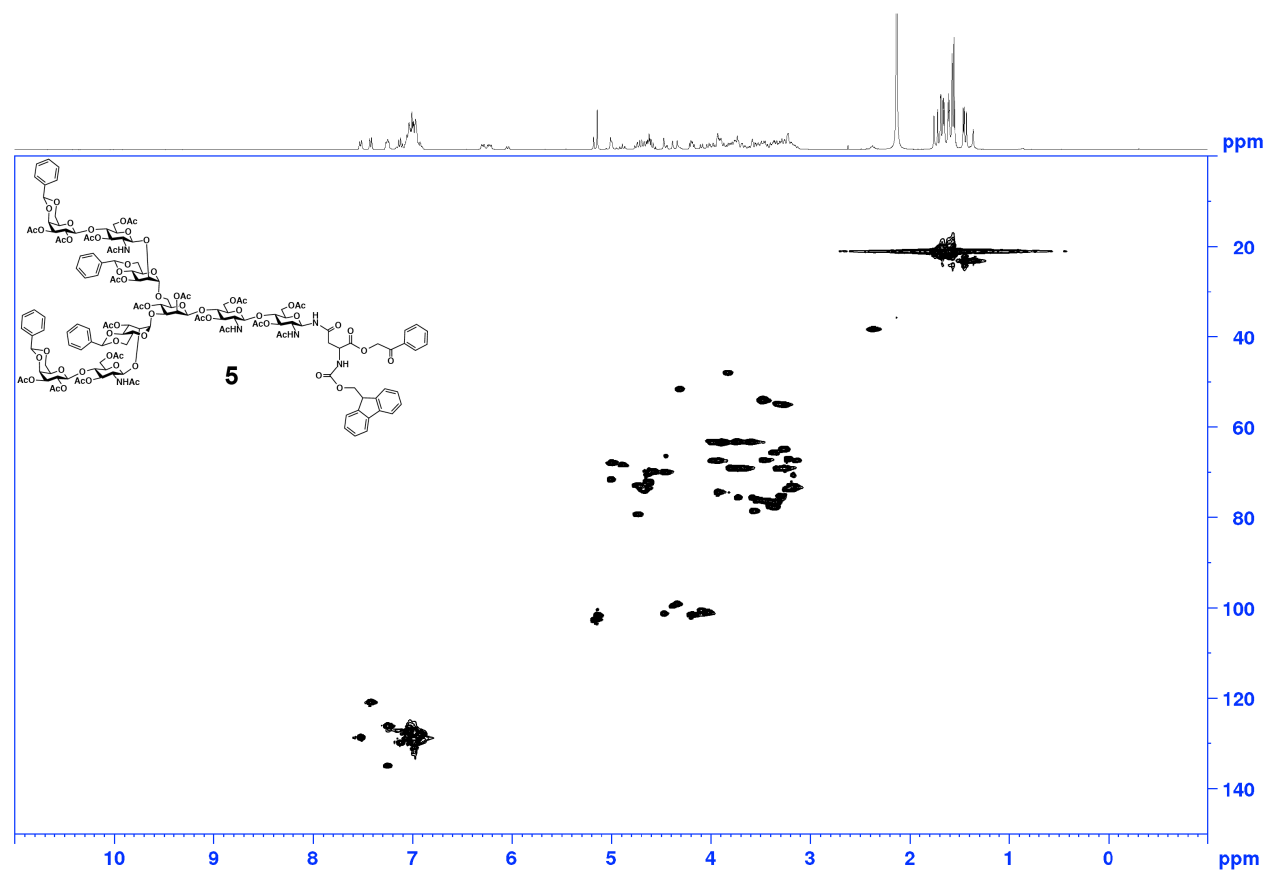

${ }^{1} \mathrm{H}$ NMR of 6

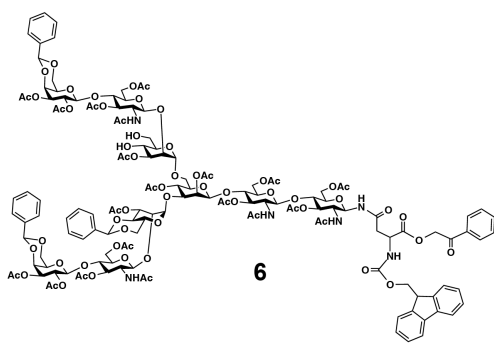


${ }^{13} \mathrm{C}$ NMR of 6

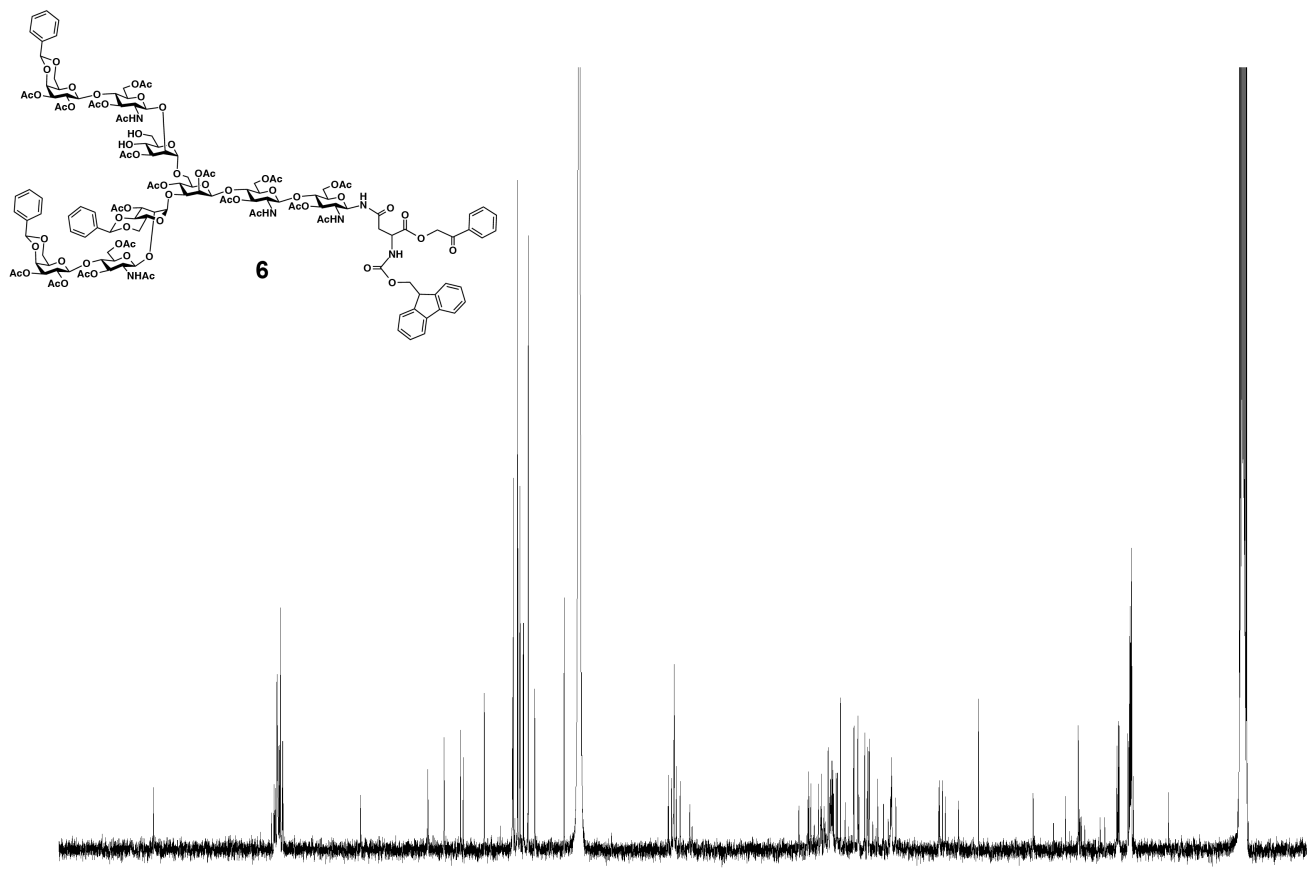

$\begin{array}{lllllllllllllllllllll}200 & 190 & 180 & 170 & 160 & 150 & 140 & 130 & 120 & 110 & 100 & 90 & 80 & 70 & 60 & 50 & 40 & 30 & 20 & 10 & \mathrm{ppm}\end{array}$

HSQC of 6

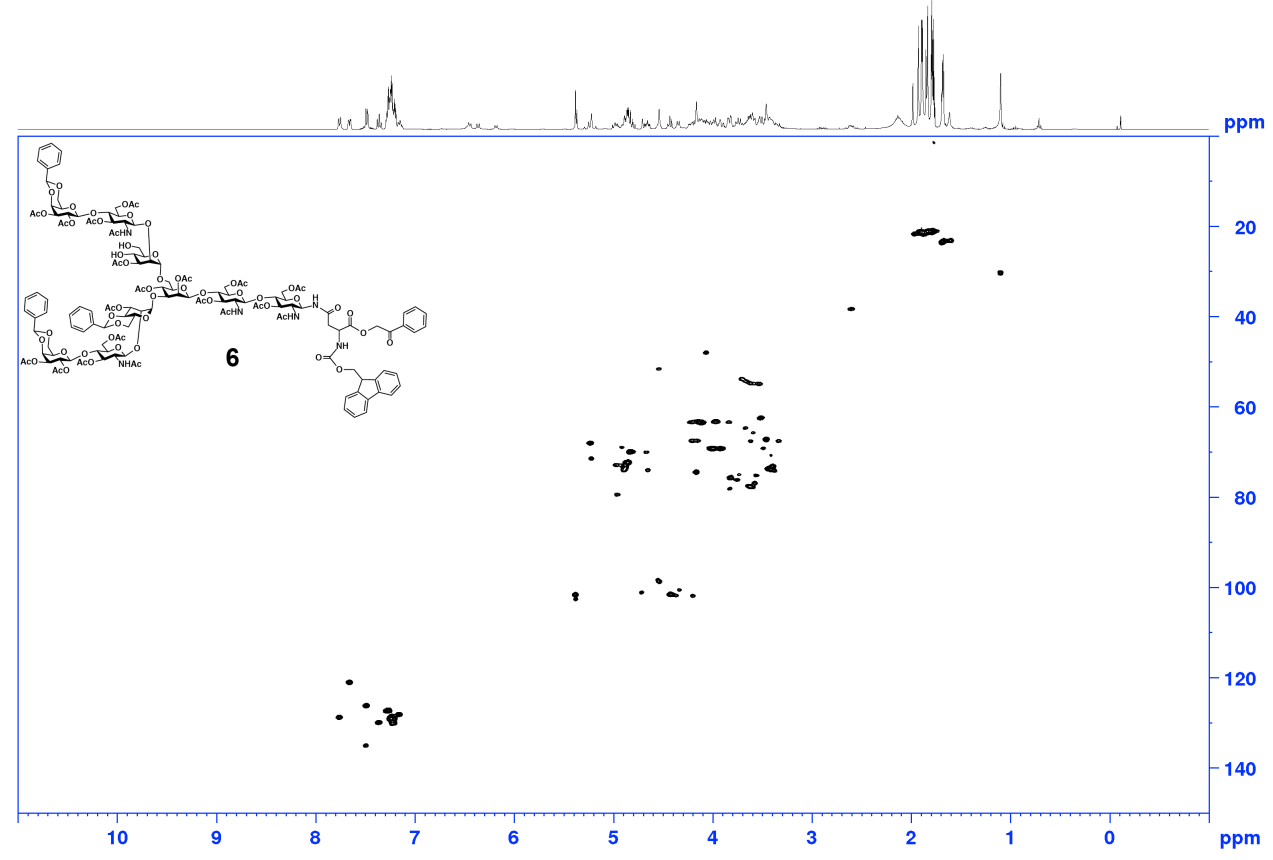


${ }^{1} \mathrm{H}$ NMR of 7

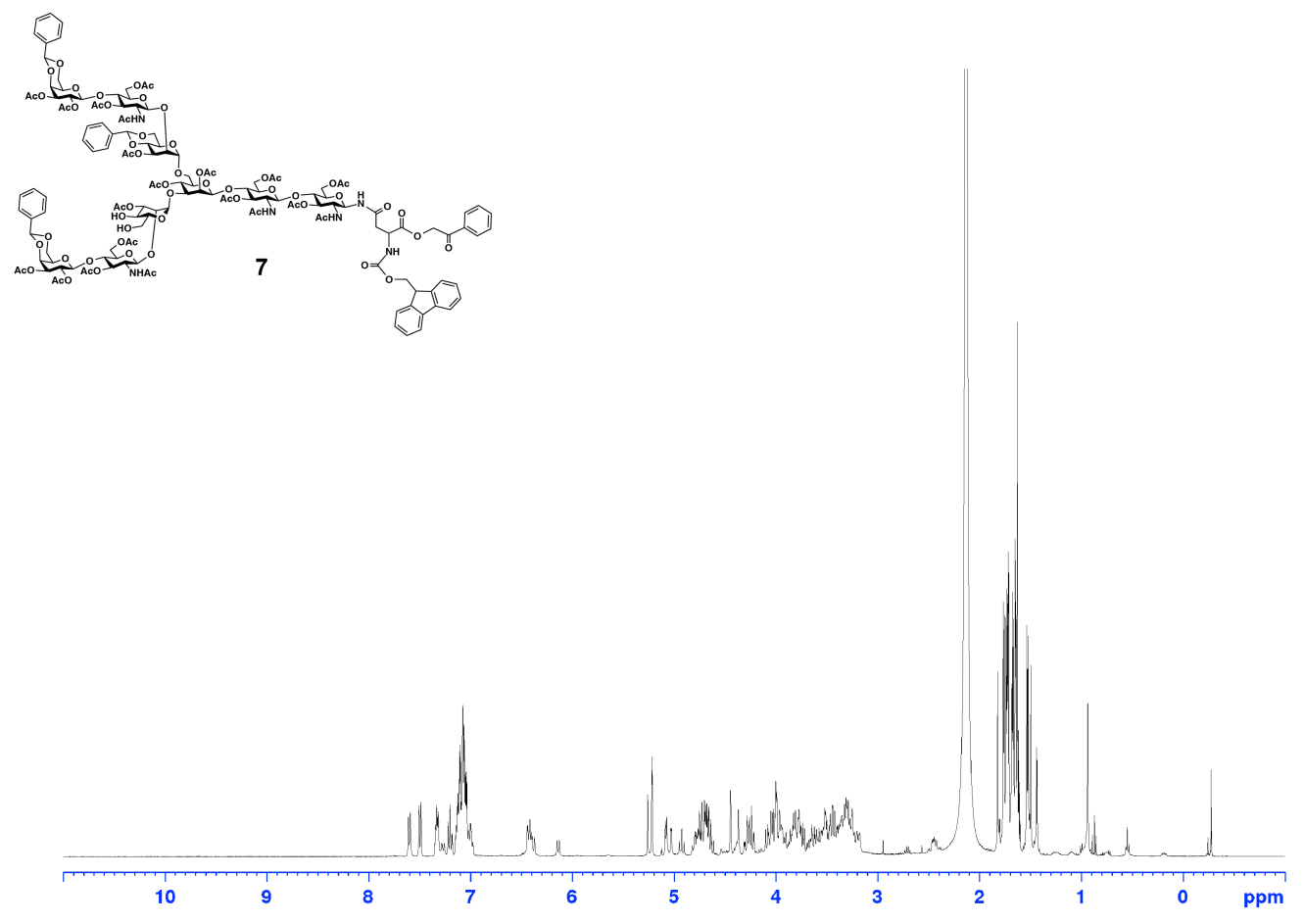

${ }^{13} \mathrm{C}$ NMR of 7

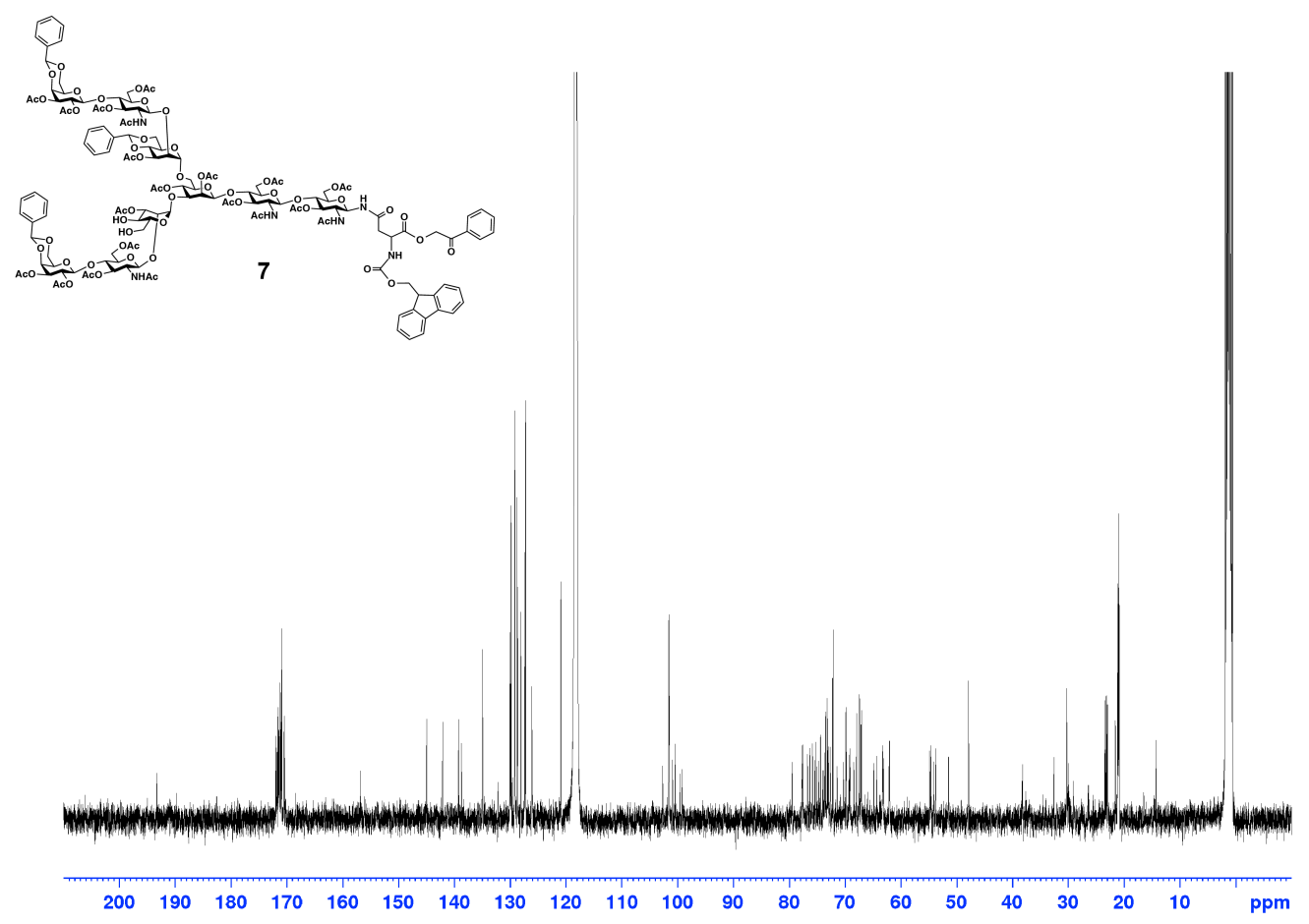


HSQC of 7

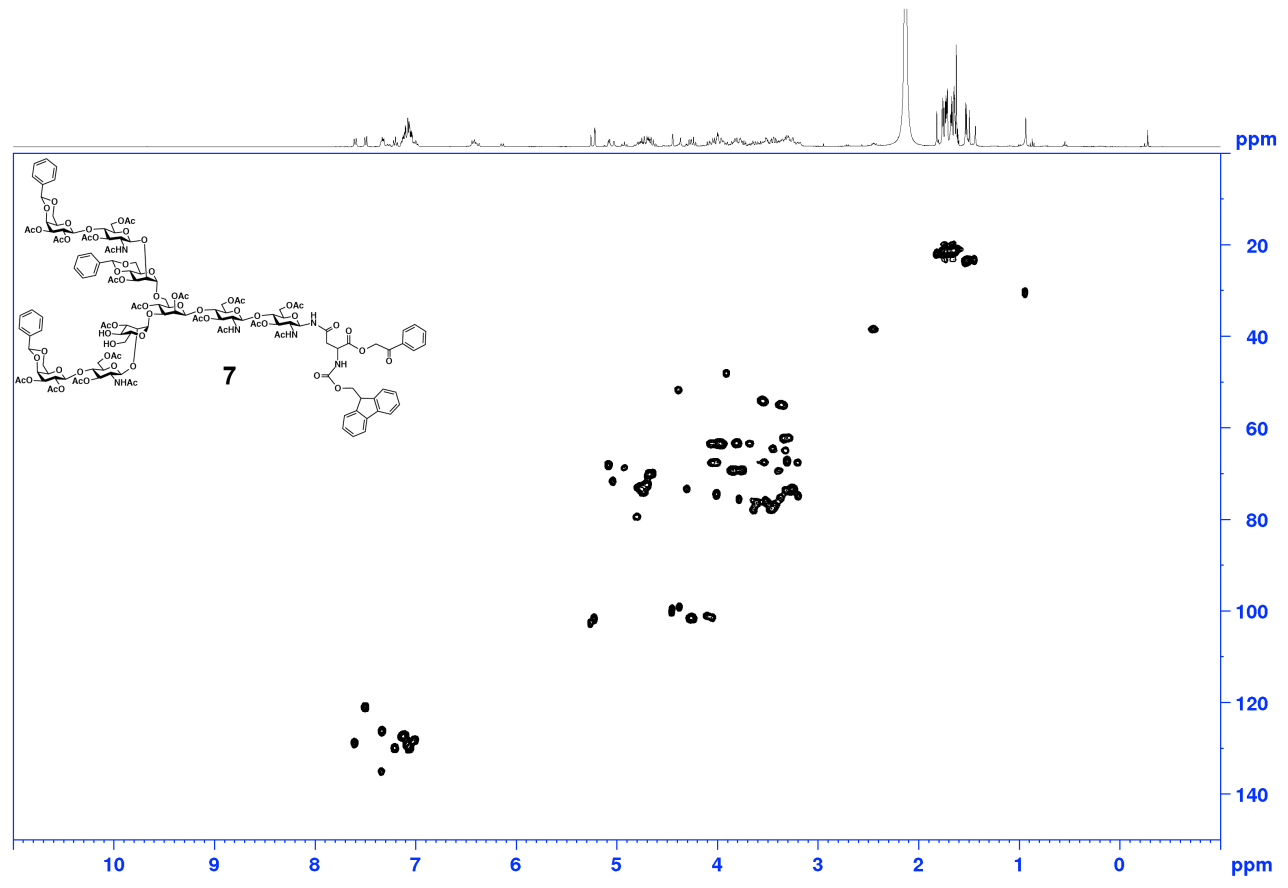


${ }^{1}$ H NMR of 8

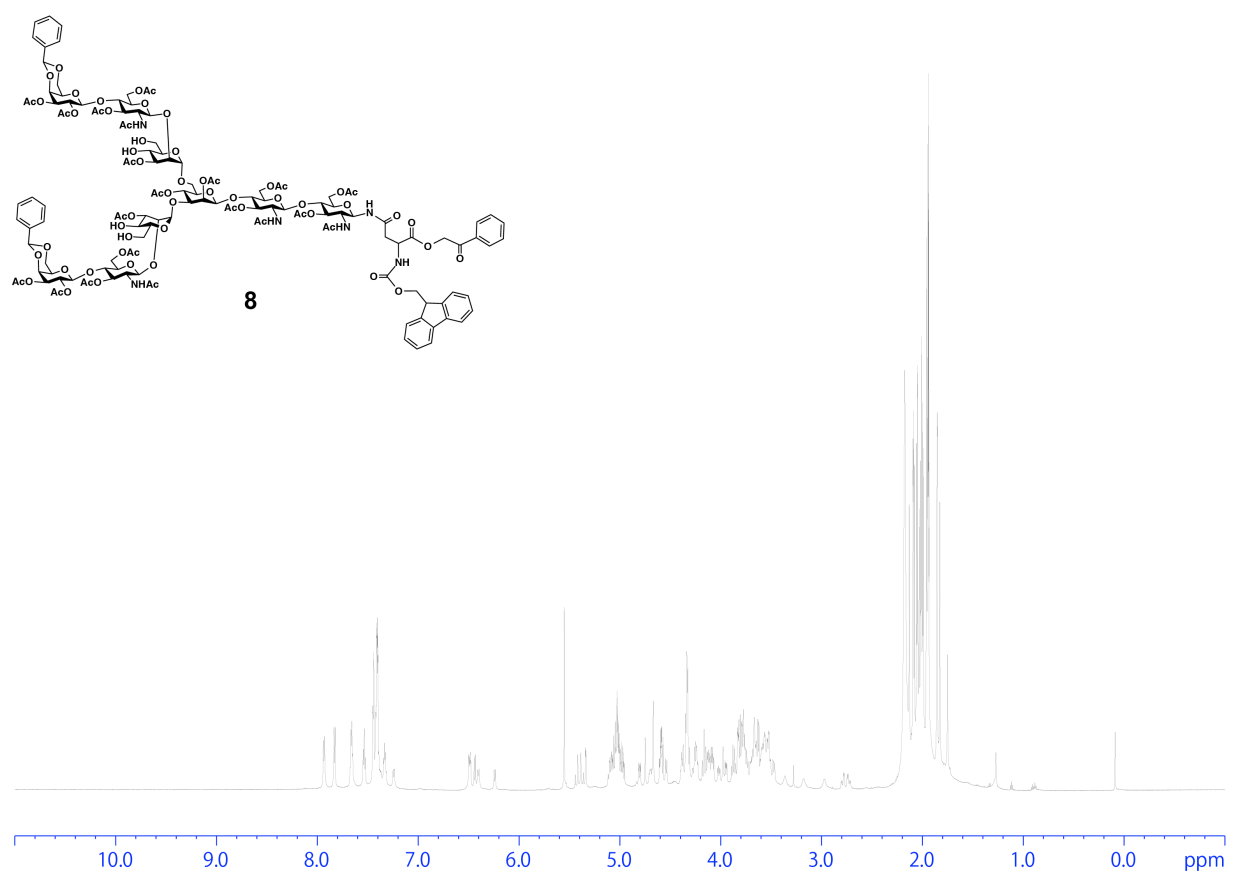

${ }^{13} \mathrm{C}$ NMR of 8

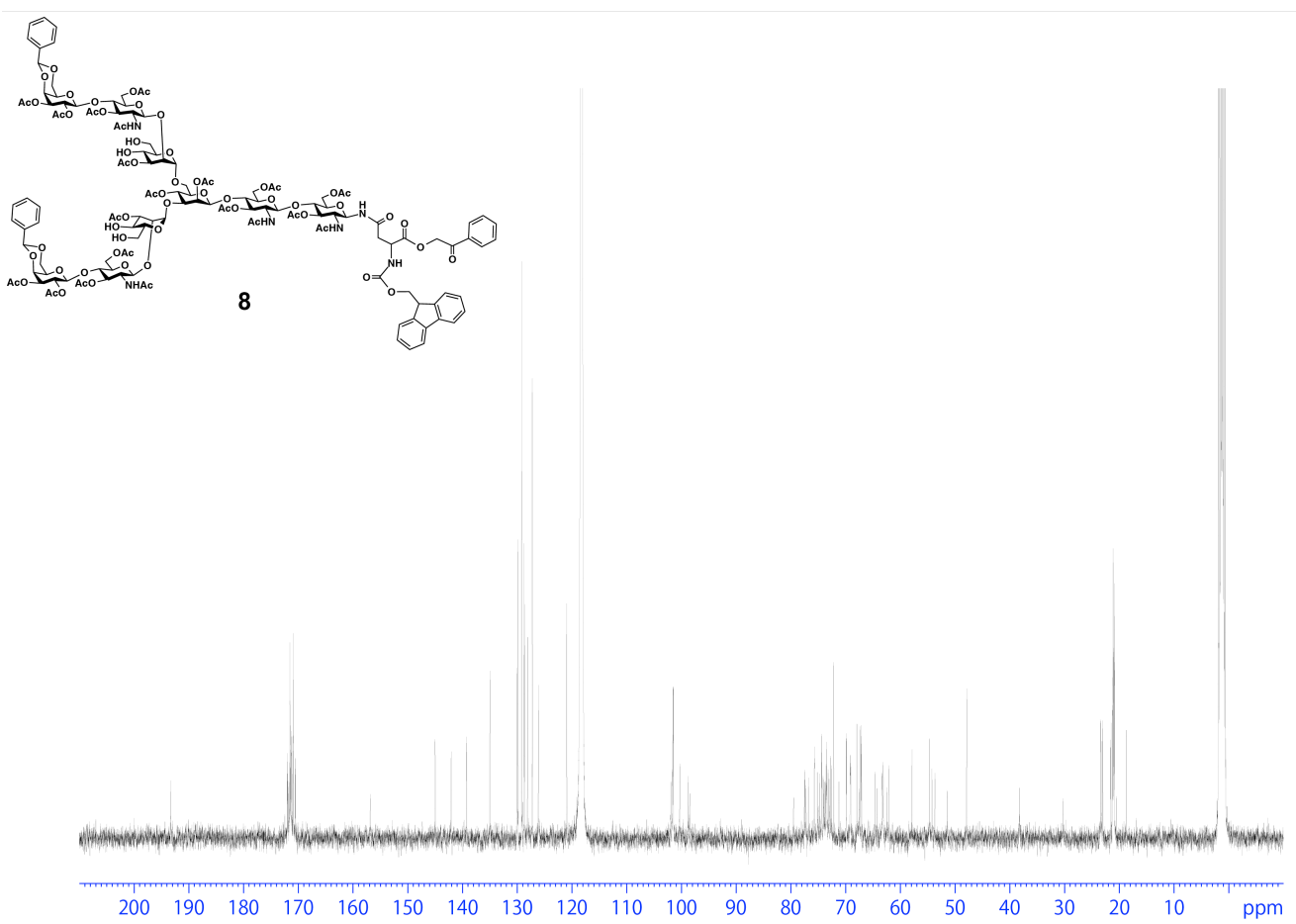


HSQC of $\mathbf{8}$

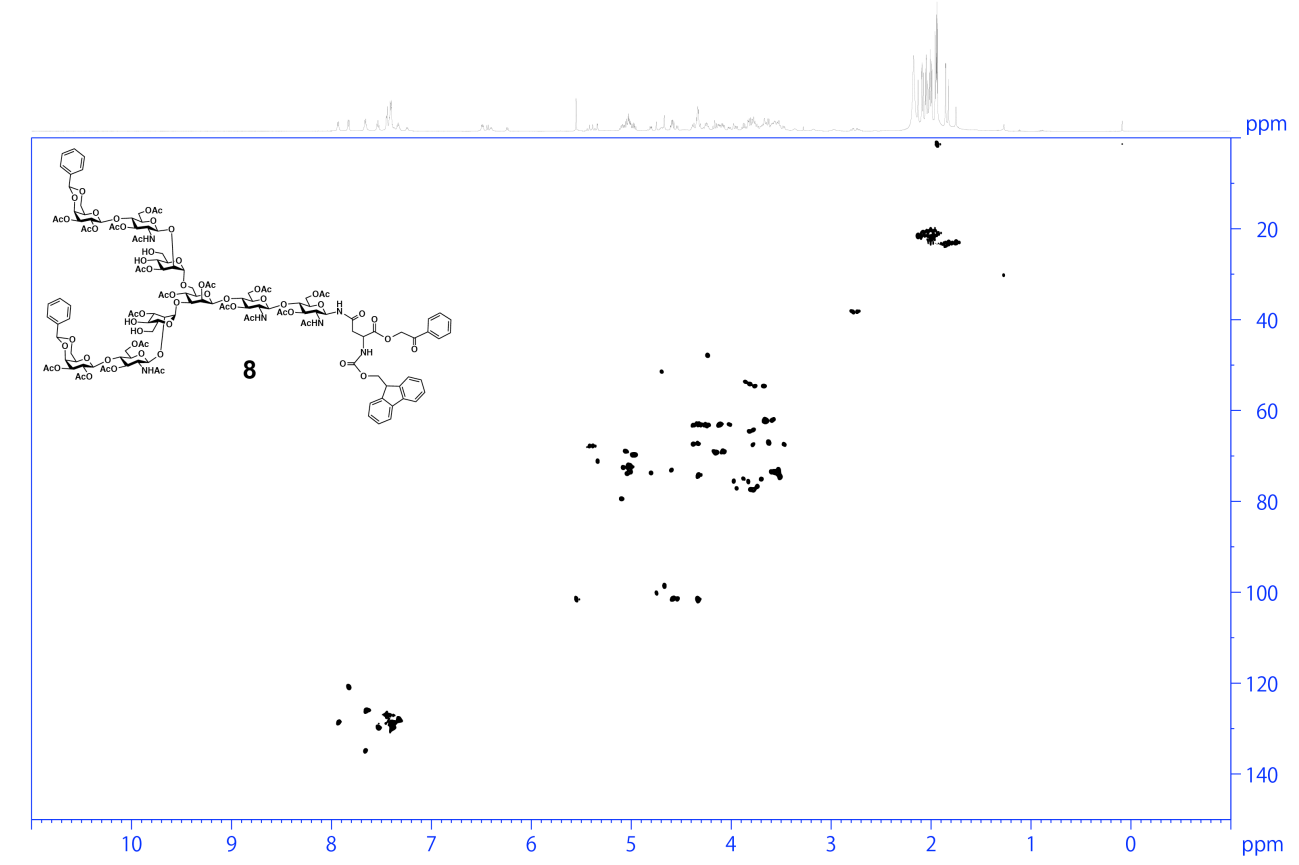


${ }^{1} \mathrm{H}$ NMR of 11

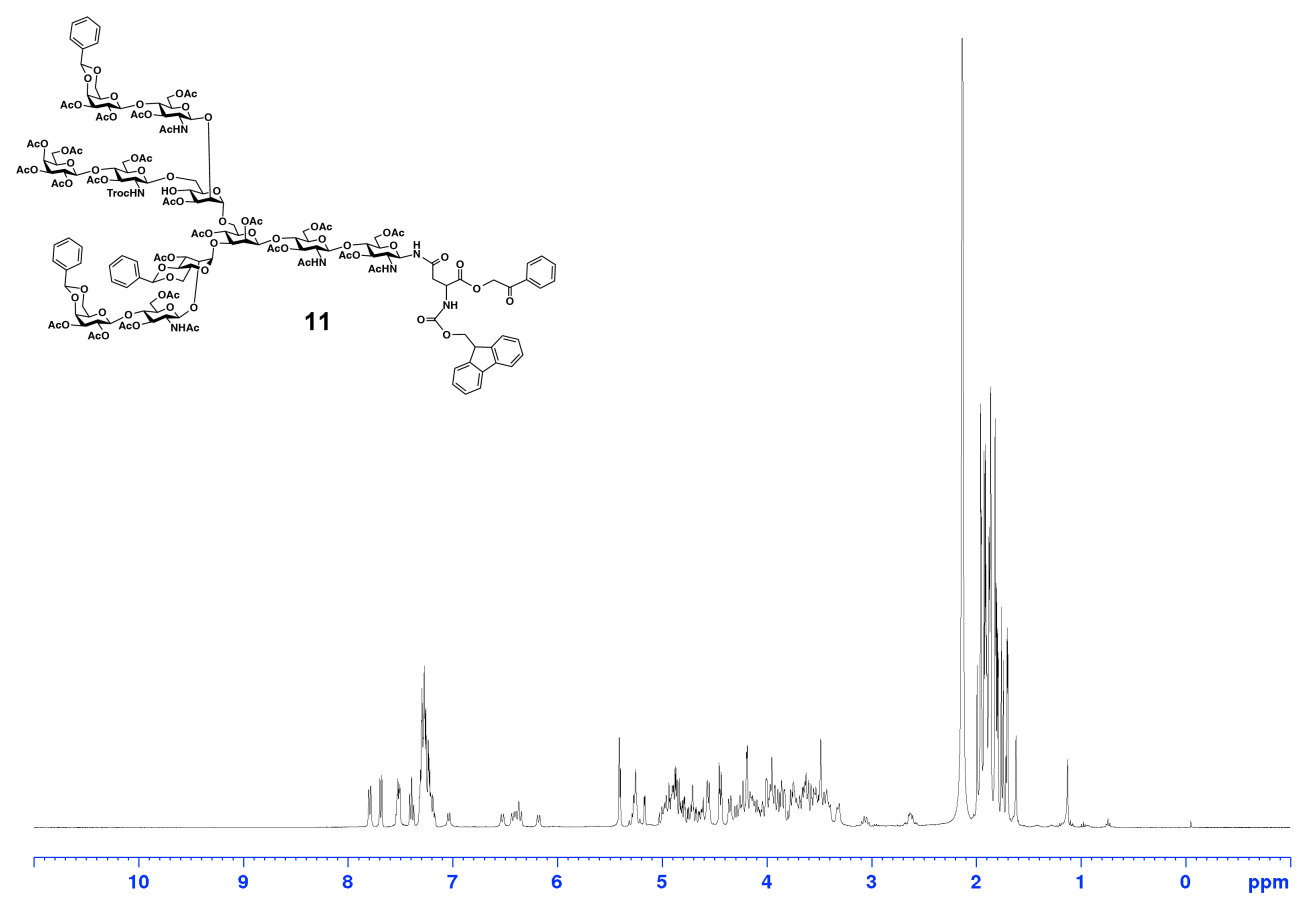

HSQC of 11

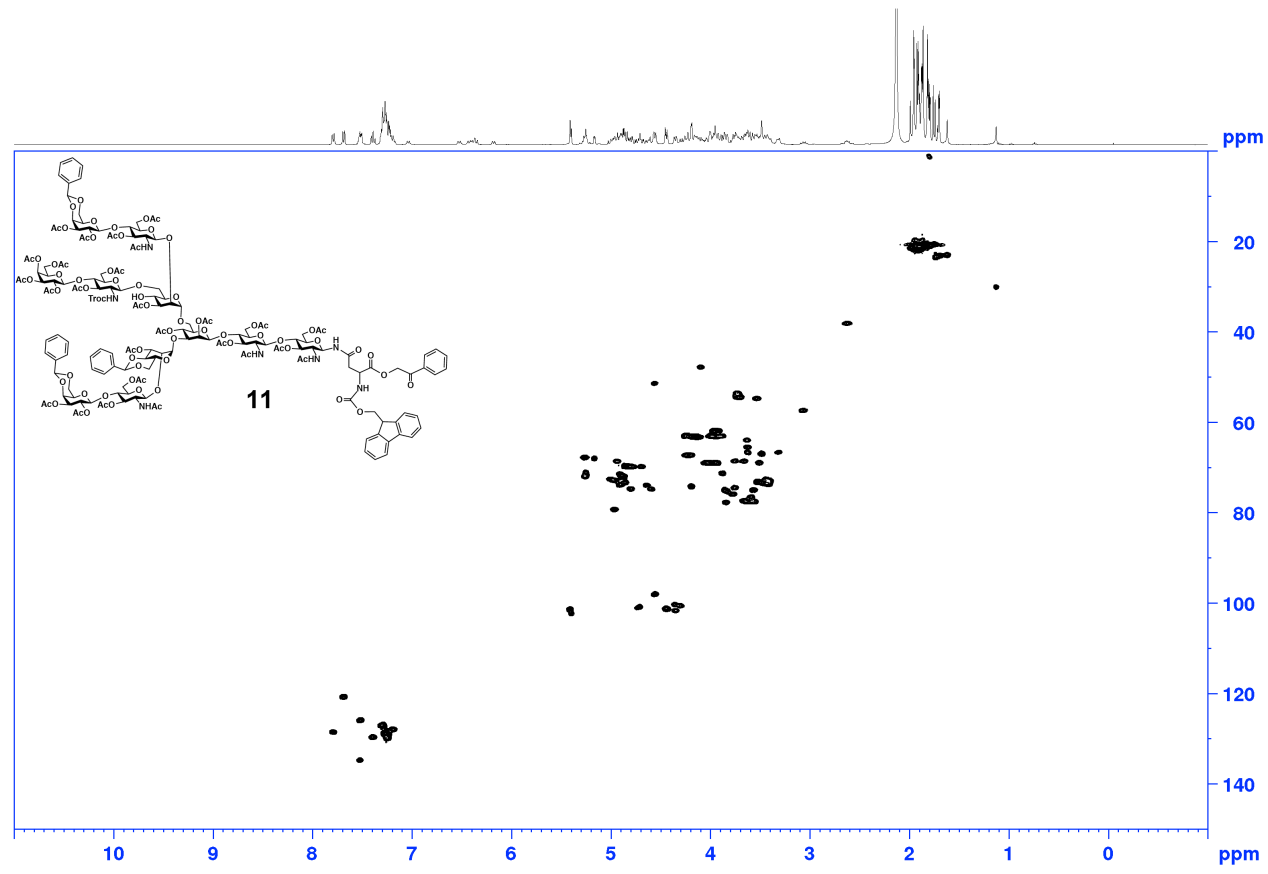




\section{${ }^{1}$ H NMR of 1}

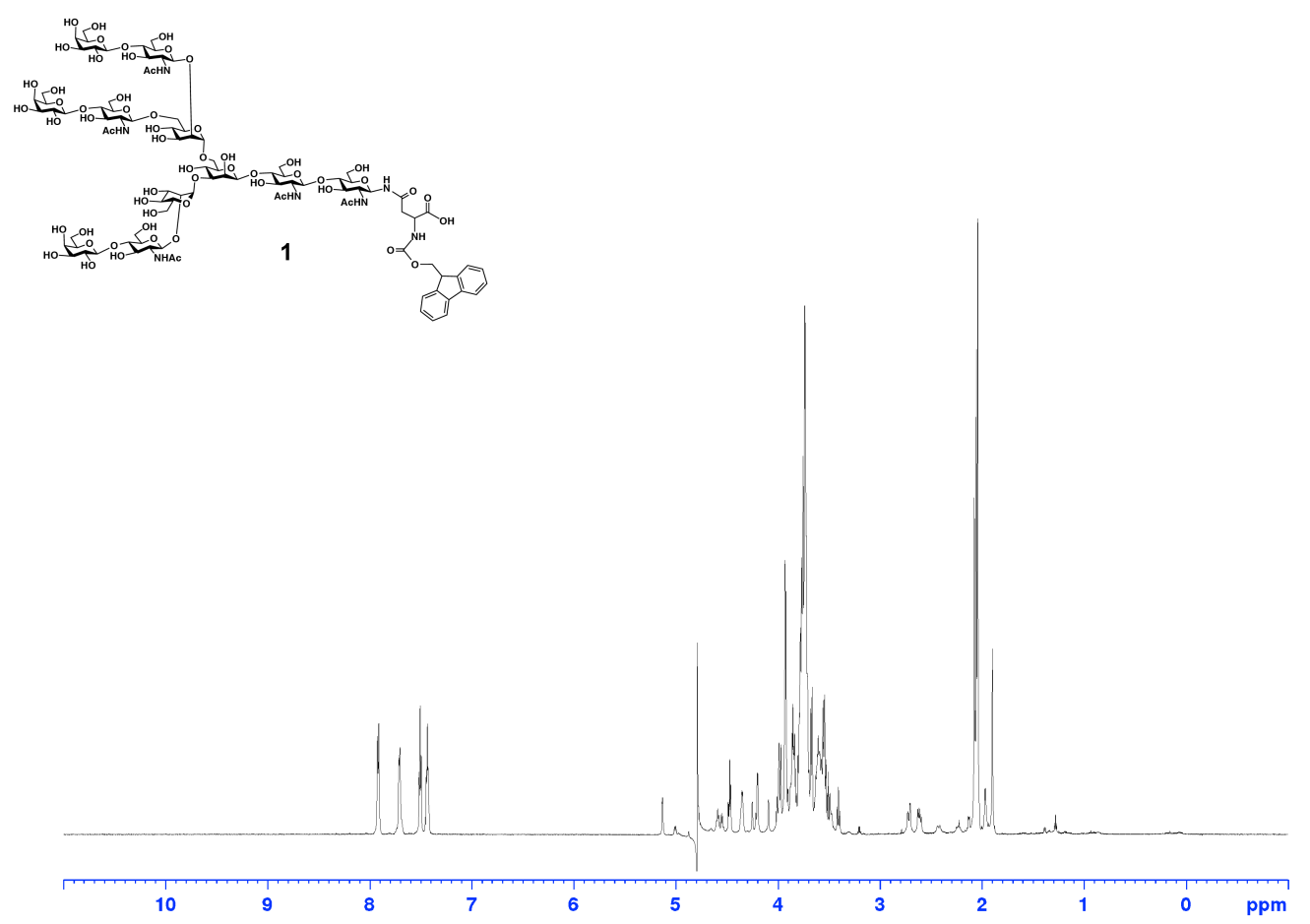

HSQC of 1

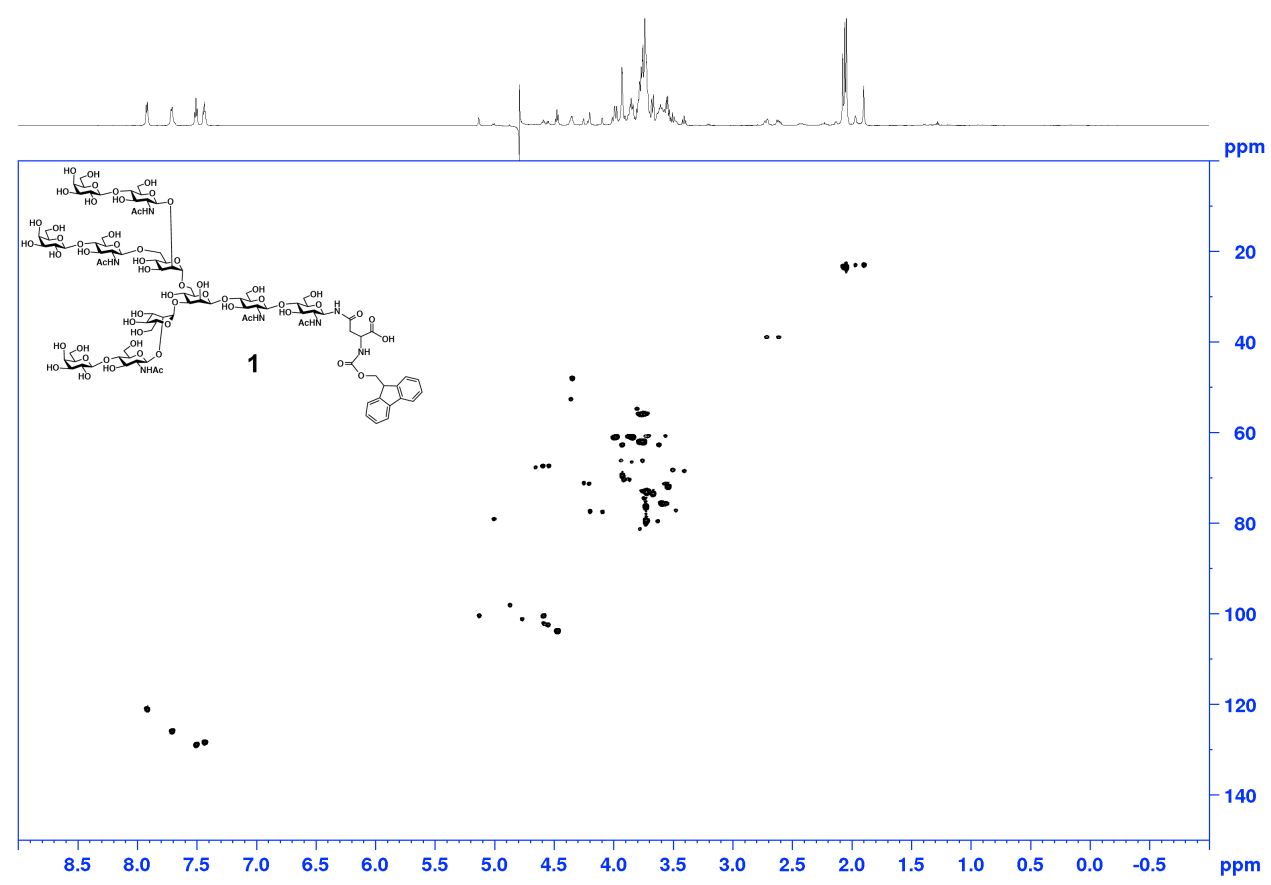


${ }^{1} \mathrm{H}$ NMR of 13 (presaturation)

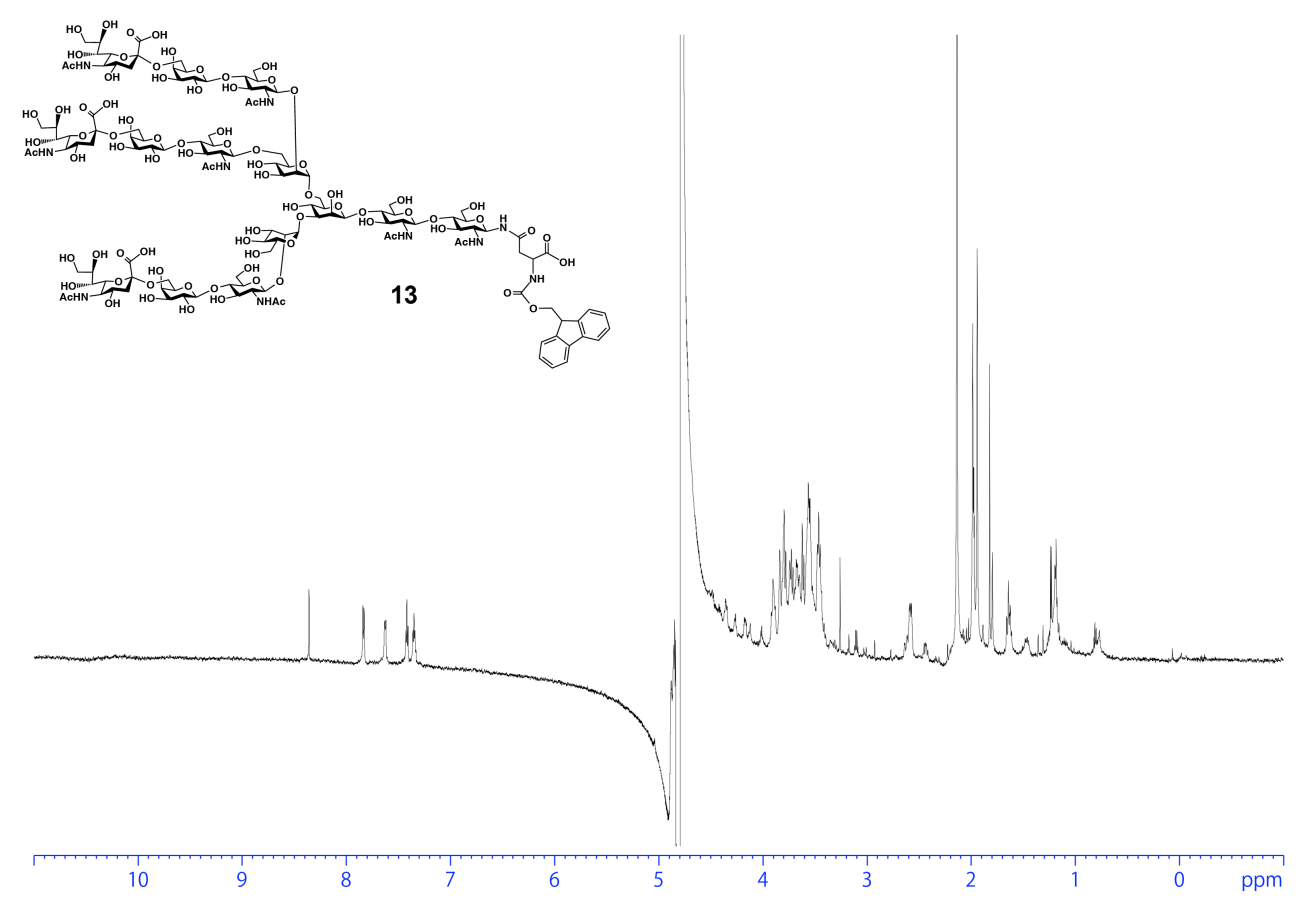

${ }^{1} \mathrm{H}$ NMR of $\mathbf{1 3}$ (watergate)

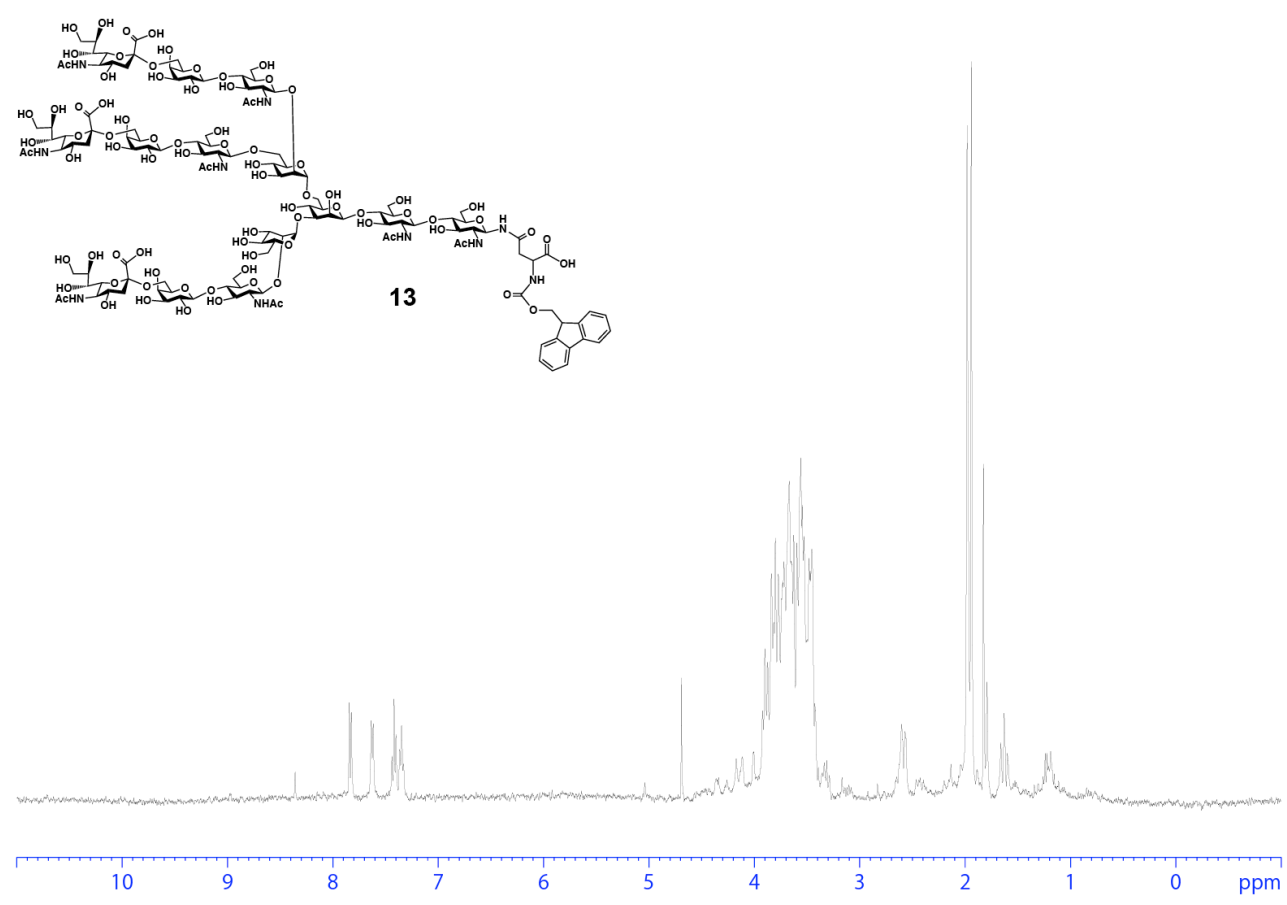


${ }^{1}$ H NMR of 9

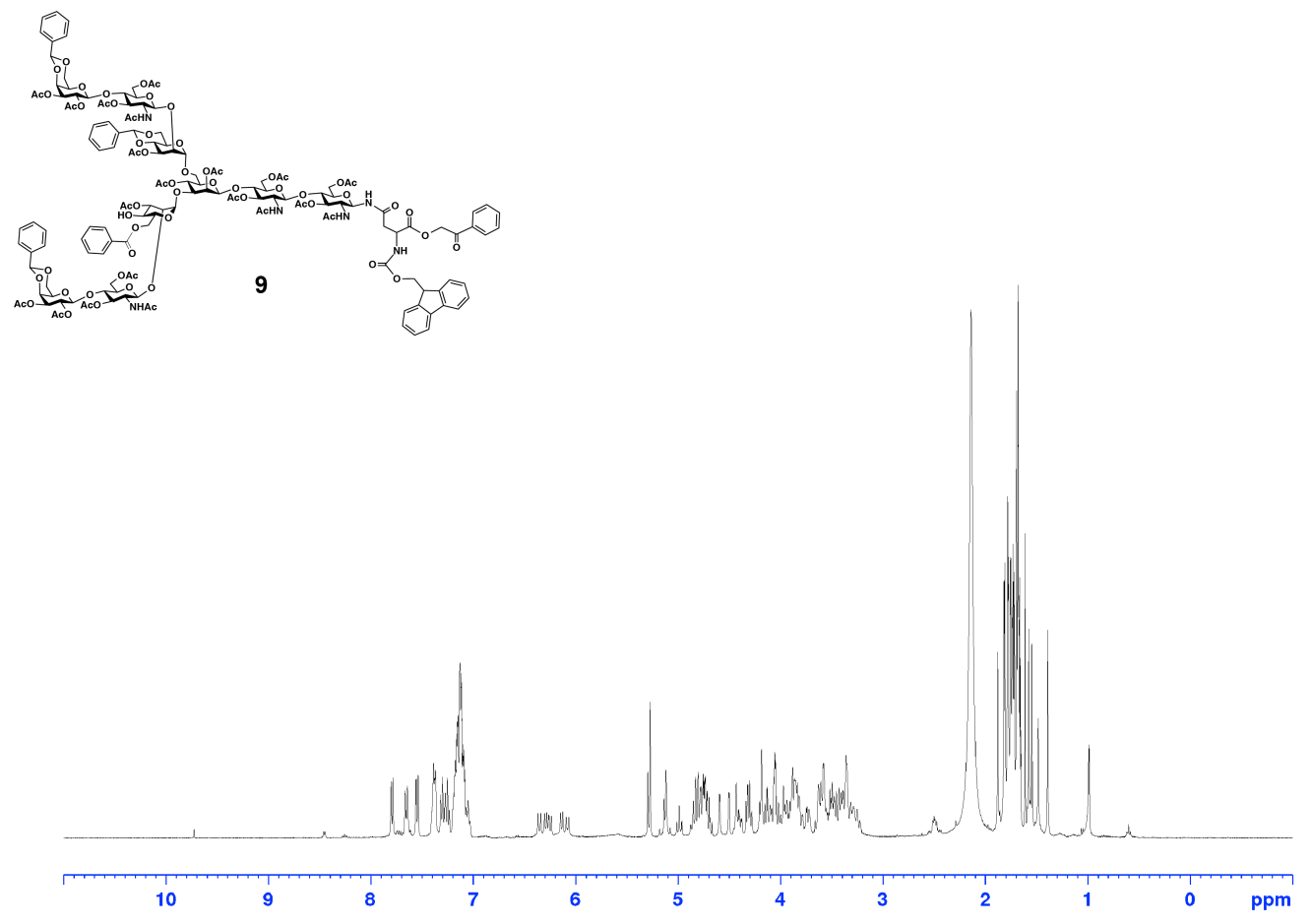

${ }^{13} \mathrm{C}$ NMR of 9

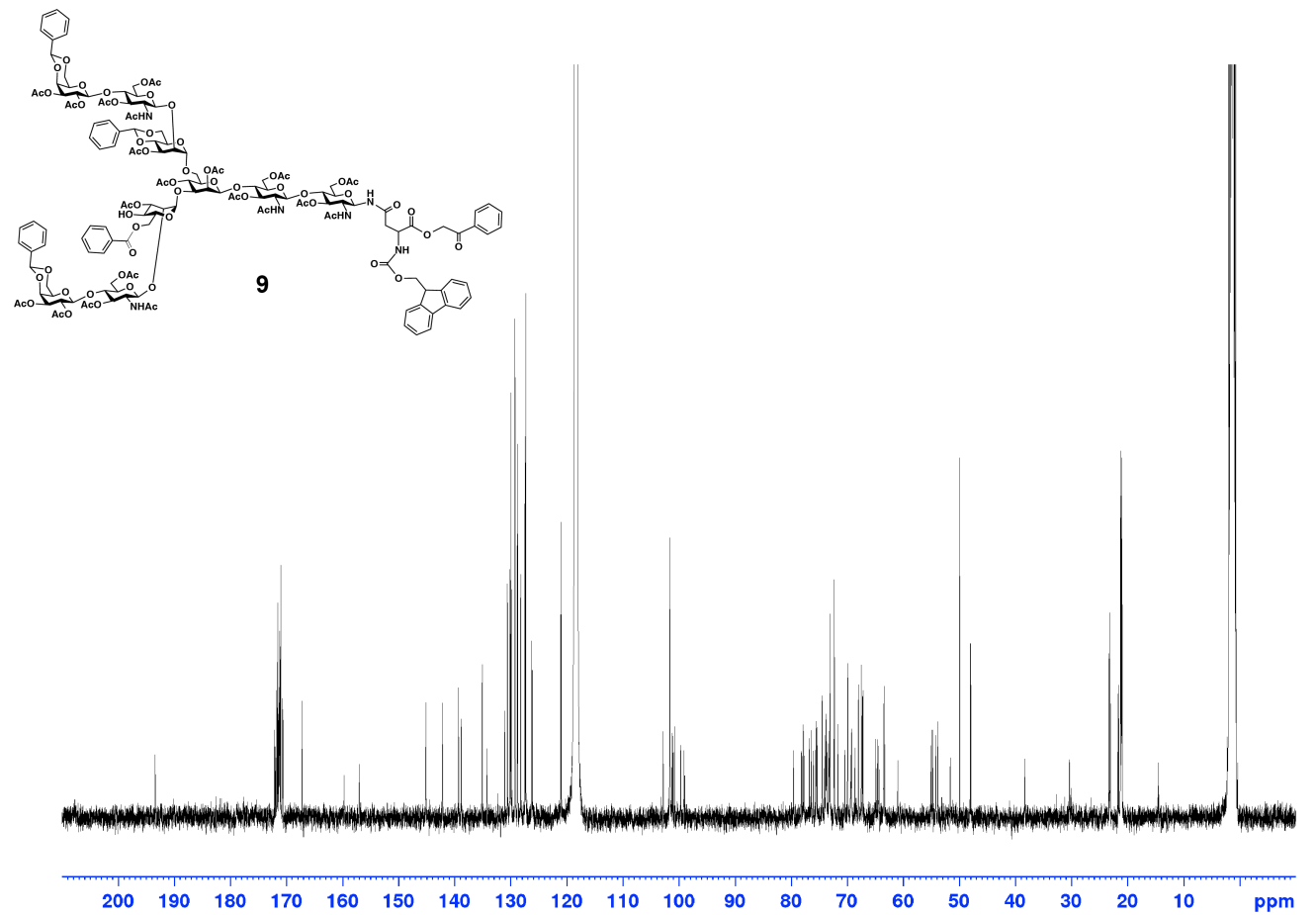


HSQC of 9

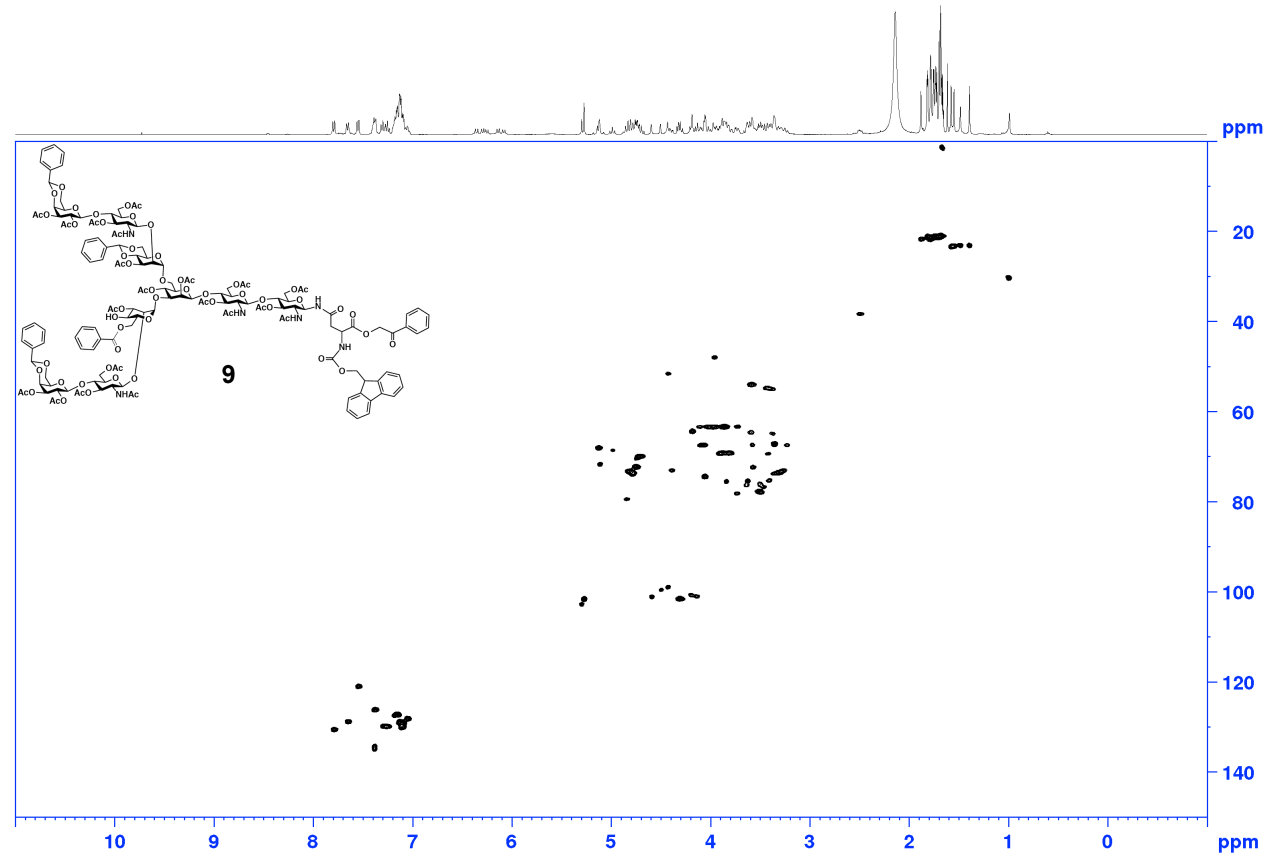


${ }^{1} \mathrm{H}$ NMR of $\mathbf{1 2}$

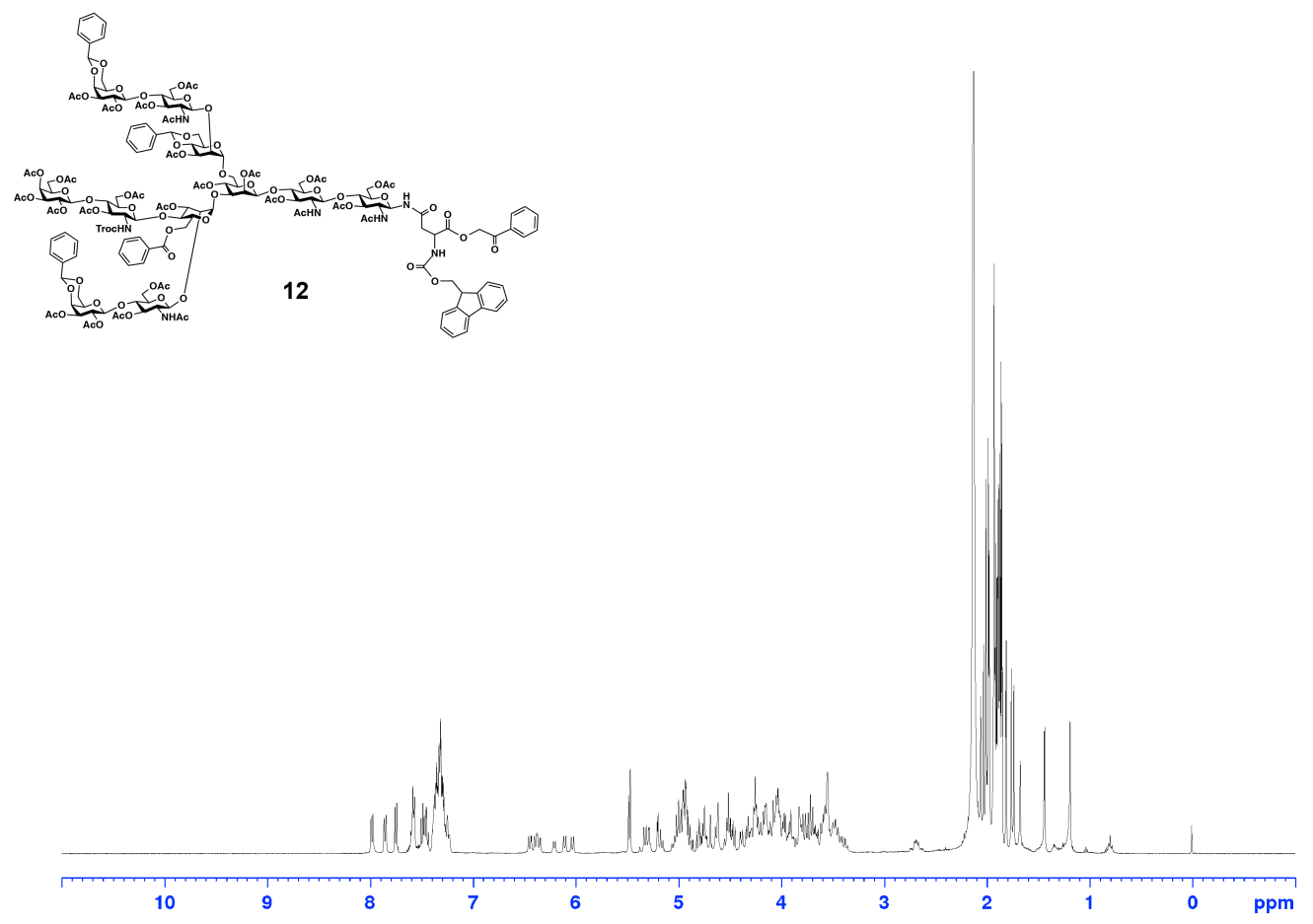

HSQC of $\mathbf{1 2}$

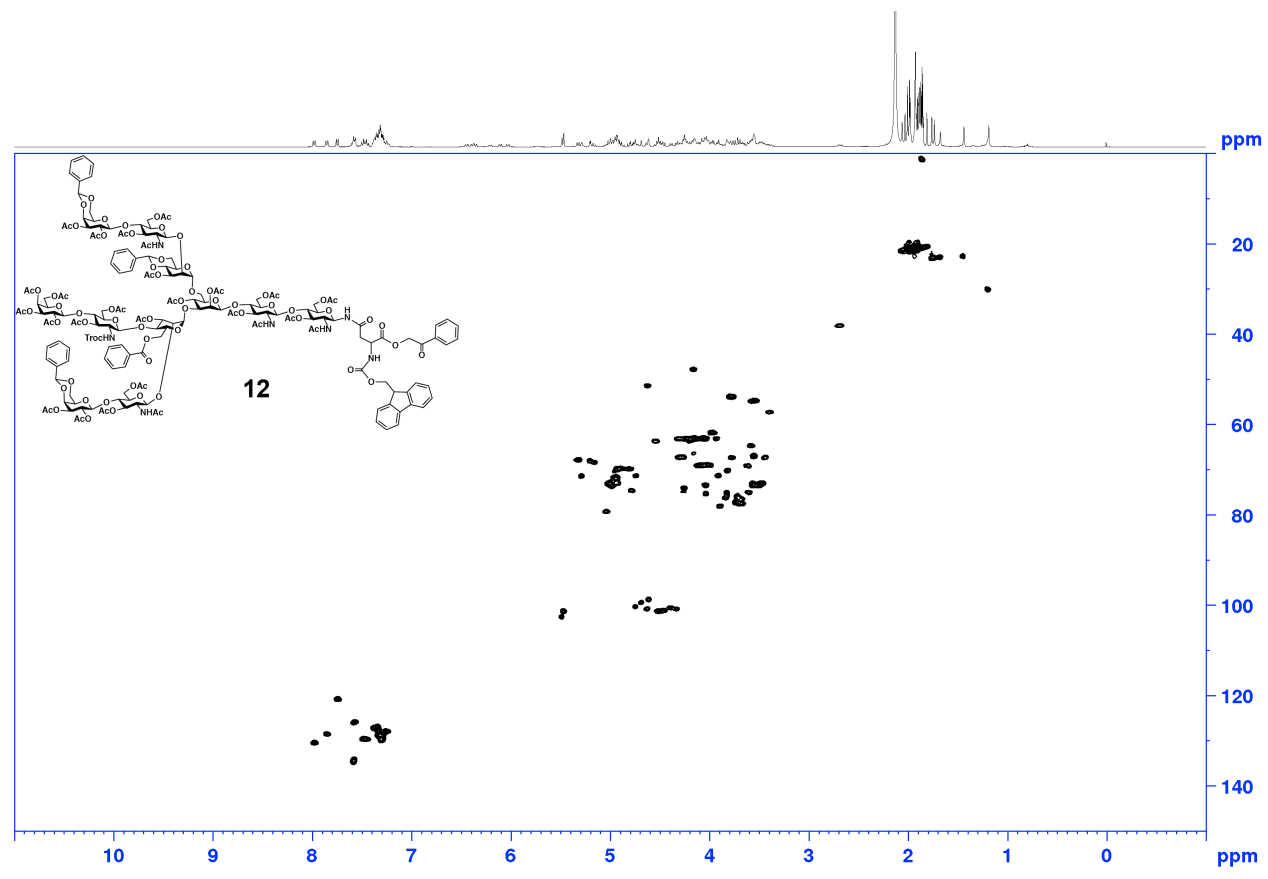




\section{${ }^{1}$ H NMR of 2}

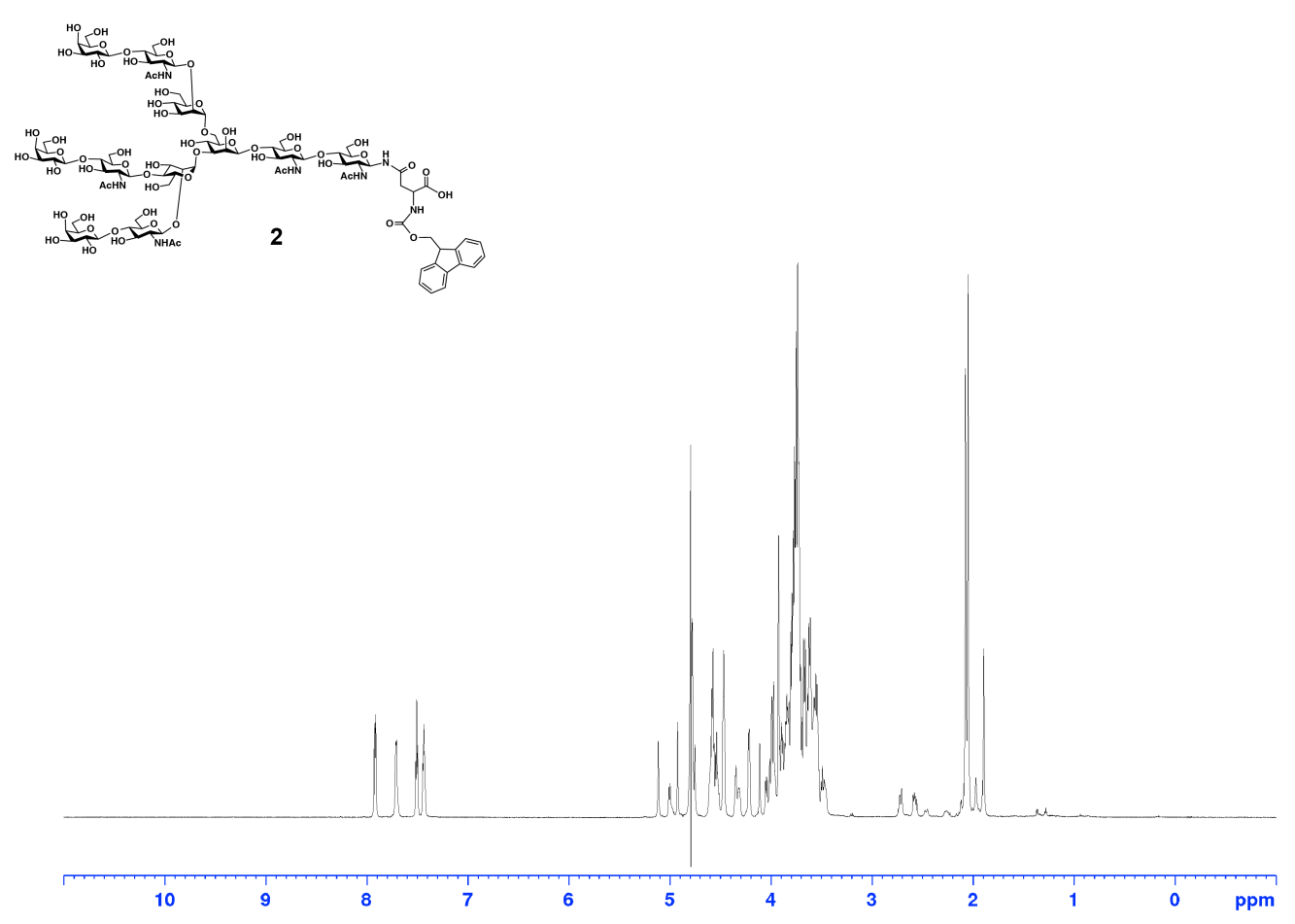

HSQC of 2

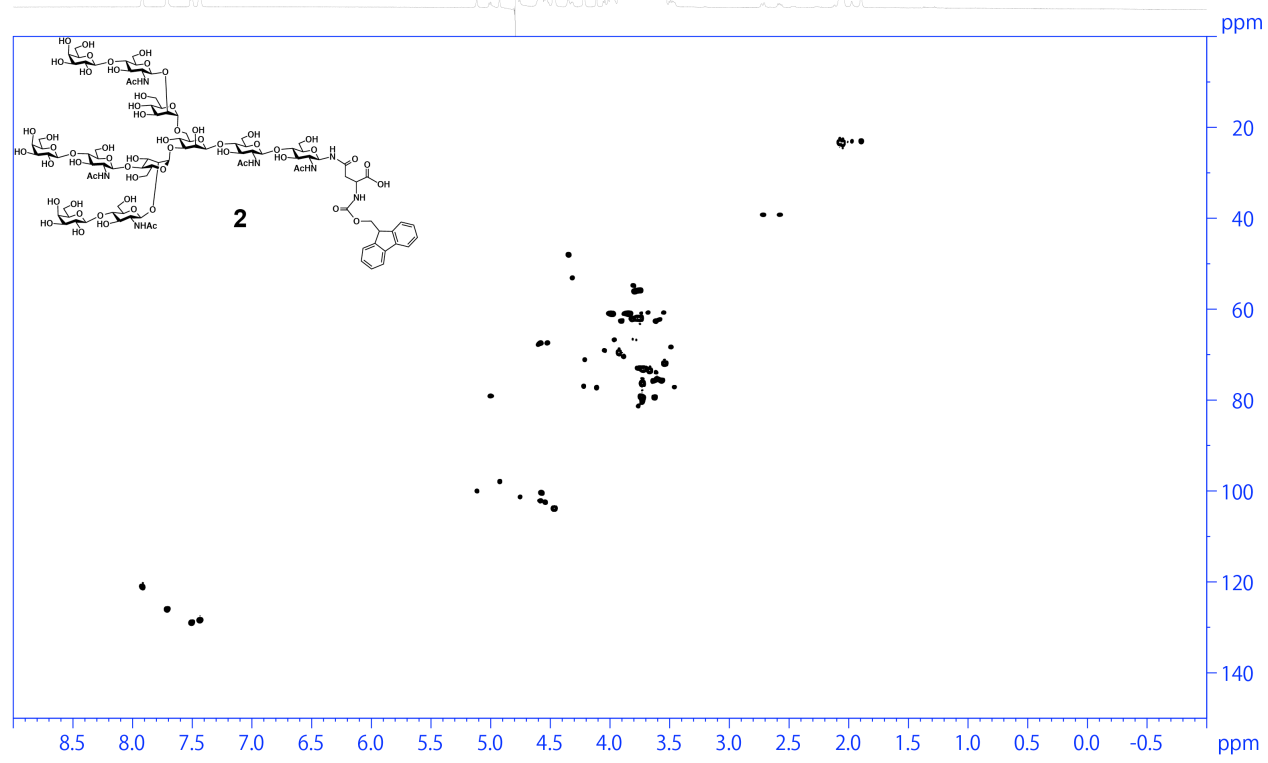


${ }^{1} \mathrm{H}$ NMR of $\mathbf{G a l}_{\mathbf{B}}$

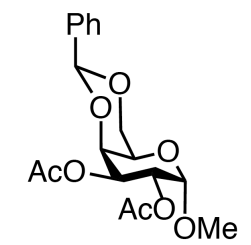

Gal(Benzylidene, diAc)

\begin{tabular}{llllllllllllll}
\hline & 10 & 9 & 8 & 7 & 6 & 5 & 4 & 3 & 2 & 1 & 0 & $\mathrm{ppm}$
\end{tabular}

${ }^{13} \mathrm{C}$ NMR of $\mathbf{G a l}_{\mathbf{B}}$

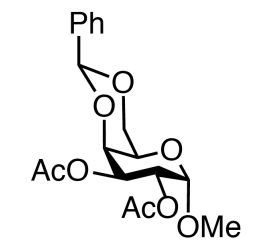

Gal(Benzylidene, diAc) 


\section{References}

(1) Kajihara, Y.; Kodama, H.; Wakabayashi, T.; Sato, K.-i.; Hashimoto, H. Carbohydr. Res. 1993, 247, 179 .

(2) Lemieux, R. U.; Abbas, S. Z.; Burzynska, M. H.; Ratcliffe, R. M. Can. J. Chem. 1982, 60, 63.

(3) Cheshev, P. E.; Kononov, L. O.; Tsvetkov, Y. E.; Shashkov, A. S.; Nifantiev, N. E. Russ. J. Bioorg. Chem. 2002, 28, 419.

(4) Yan, F.; Mehta, S.; Eichler, E.; Wakarchuk, W. W.; Gilbert, M.; Schur, M. J.; Whitfield, D. M. J. Org. Chem. 2003, 68, 2426.

(5) Kajihara, Y.; Suzuki, Y.; Yamamoto, N.; Sasaki, K.; Sakakibara, T.; Juneja, L. R. Chem.-Eur. J. 2004, 10, 971.

(6) Sato, H.; Fukae, K.; Kajihara, Y. Carbohydr. Res. 2008, 343, 1333.

(7) Jeanloz, R. W.; Jeanloz, D. A. J. Am. Chem. Soc. 1957, 79, 2579.

(8) Horton, D.; Luetzow, A. E. Carbohydr. Res. 1968, 7, 101.

(9) Poláková, M.; Roslund, M. U.; Ekholm, F. S.; Saloranta, T.; Leino, R. Eur. J. Org. Chem. 2009, 2009, 870 . 